\title{
Short-lived Nuclei in the Early Solar System: Possible AGB Sources
}

\author{
G. J. Wasserburg \\ The Lunatic Asylum, Division of Geological and Planetary Sciences, California \\ Institute of Technology, Pasadena, CA 91125 \\ M. Busso \\ Department of Physics, University of Perugia, via Pascoli, Perugia, Italy, 06123 \\ R. Gallino \\ Department of General Physics and Sezione INFN, University of Torino, via P. \\ Giuria 1, Torino, Italy, 10125; also Centre for Stellar and Planetary Astrophysics, \\ School of Mathematical Sciences, Monash University, 3800 Victoria, Australia \\ K. M. Nollett \\ Physics Division, Argonne National Laboratory, Argonne, IL 60439-4843
}




\section{Abstract}

The abundances of short-lived radionuclides in the early solar system (ESS) are reviewed, as well as the methodology used in determining them. These results are compared with the inventory estimated for a uniform galactic production model. It is shown that, to within a factor of two, the observed abundances of ${ }^{238} \mathrm{U},{ }^{235} \mathrm{U}$, ${ }^{232} \mathrm{Th},{ }^{244} \mathrm{Pu},{ }^{182} \mathrm{Hf},{ }^{146} \mathrm{Sm}$, and ${ }^{53} \mathrm{Mn}$ are roughly compatible with long-term galactic nucleosynthesis. ${ }^{129} \mathrm{I}$ is an exception, with an ESS inventory much lower than expected from uniform production. The isotopes ${ }^{107} \mathrm{Pd},{ }^{60} \mathrm{Fe},{ }^{41} \mathrm{Ca},{ }^{36} \mathrm{Cl},{ }^{26} \mathrm{Al}$, and ${ }^{10} \mathrm{Be}$ require late addition to the protosolar nebula. ${ }^{10} \mathrm{Be}$ is the product of energetic particle irradiation of the solar system as most probably is ${ }^{36} \mathrm{Cl}$. Both of these nuclei appear to be present when ${ }^{26} \mathrm{Al}$ is absent. A late injection by a supernova (SN) cannot be responsible for most of the short-lived nuclei without excessively producing ${ }^{53} \mathrm{Mn}$; it can however be the source of ${ }^{53} \mathrm{Mn}$ itself and possibly of ${ }^{60} \mathrm{Fe}$. If a late SN injection is responsible for these two nuclei, then there remains the problem of the origin of ${ }^{107} \mathrm{Pd}$ and several other isotopes. Emphasis is given to an AGB star as a source of many of the nuclei, including ${ }^{60} \mathrm{Fe}$; this possibility is explored with a new generation of stellar models. It is shown that if the dilution factor (i.e. the ratio of the contaminating mass to the solar parental cloud mass) is $f_{0} \sim 4 \times 10^{-3}$, a reasonable representation for many nuclei is obtained; this requires that $\left({ }^{60} \mathrm{Fe} /{ }^{56} \mathrm{Fe}\right)_{E S S}$ $\sim 10^{-7}$ to $2 \times 10^{-6}$. The nuclei produced by an AGB source do not include ${ }^{53} \mathrm{Mn}$, ${ }^{10} \mathrm{Be}$ or ${ }^{36} \mathrm{Cl}$ if it is very abundant. The role of irradiation is discussed with regard to ${ }^{26} \mathrm{Al},{ }^{36} \mathrm{Cl}$ and ${ }^{41} \mathrm{Ca}$, and the estimates of bulk solar abundances of these isotopes are commented on. The conflict between various scenarios is emphasized as well as the current absence of an astrophysically plausible global interpretation for all the existing data. Examination of abundances for the actinides indicates that a quiescent interval of $\sim 10^{8}$ years is required for actinide group production. This is needed in order to explain the data on ${ }^{244} \mathrm{Pu}$ and the new bounds on ${ }^{247} \mathrm{Cm}$. Because this quiescent interval is not compatible with the ${ }^{182} \mathrm{Hf}$ data, a separate type of $r$-process event is needed for at least the actinides, distinct from the two types that have previously been identified. The apparent coincidence of the ${ }^{129} \mathrm{I}$ and trans-actinide time scales suggests that the last heavy $r$ contribution was from an $r$-process that produced very heavy nuclei but without fission recycling so that the yields at $\mathrm{Ba}$ and below (including I) were governed by fission.

Key words: Solar abundances - Short-lived nuclei - Nucleosynthesis - Solar System formation - Isotopic Anomalies - Stars: AGB - Stars: Supernovae.

Email addresses: isotopes@gps.caltech.edu (G. J. Wasserburg), maurizio.busso@fisica.unipg.it (M. Busso), gallino@ph.unito.it (R. Gallino), nollett@anl.gov (K. M. Nollett). 


\section{Introduction}

More than forty years ago John Reynolds [1] at Berkeley announced the discovery of an excess of ${ }^{129} \mathrm{Xe}$ in a meteorite $\left({ }^{129} \mathrm{Xe}^{*}\right)$ and suggested that it could be ascribed to the in situ radioactive decay of ${ }^{129} \mathrm{I}(\bar{\tau}=23 \mathrm{Myr})$. This was proven later, through a demonstration that excesses of ${ }^{129} \mathrm{Xe}^{*}$ are directly correlated with stable ${ }^{127} \mathrm{I}[2]$. These isotopes of iodine are $r$-process products, attributed to supernovae. It was immediately recognized [3] that the observed abundance of ${ }^{129} \mathrm{I}$ could be ascribed to the long-term production of $r$-process nuclei in the Galaxy, provided the solar system material had been isolated from the interstellar medium for about $10^{8}$ years. We can thus notice how basic questions on the presence of "live" radioactivities in the Early Solar System (hereafter ESS) were associated with supernova sources since the beginning of modern research efforts. However, proper answers for the sources of nuclei have then been looked for in many works and remain an open issue today.

The search for radionuclides other than ${ }^{129}$ I was, broadly speaking, not successful for a long period of time. There were many negative or failed or erroneous efforts. Critical advances in analytical techniques would govern the progress. These advances involved great improvements in high-precision, high-sensitivity mass spectrometry, analytical microchemistry, and sample preparation. Crucial for all subsequent progress was the fall of the Allende meteorite in 1969. This fall was contemporaneous with major efforts in some laboratories to prepare for lunar samples to be returned by the Apollo missions. The Allende fall made it possible to sample old materials from the solar nebula including early refractory condensates, the Calcium and Aluminum Inclusions (CAIs) [4] [5] [6]. Measurements of ${ }^{129} \mathrm{Xe}^{*}$ were also done on these inclusions and gave the same results as found for normal chondrites [7]. These early-formed samples in Allende then also yielded quantitative evidence of the existence of radioactive nuclei with a much shorter meanlife. In particular, ${ }^{26} \mathrm{Al}(\bar{\tau}=1.03 \mathrm{Myr})$ had been sought earlier in meteoritic material using precise methods [8] but had not been found in the available meteoritic or lunar samples. However, with the fall of Allende, the important discovery by Clayton, Grossman and Mayeda of large oxygen anomalies that represented substantial shifts in the isotopic abundances of a major element [9], and the demonstration of exceedingly primitive $\mathrm{Sr}$ - very low ${ }^{87} \mathrm{Sr} /{ }^{86} \mathrm{Sr}$ ratios with no enhancements due to ${ }^{87} \mathrm{Rb}$ decay in some refractory inclusions - led to the possibility that ${ }^{26} \mathrm{Al}$ should be looked for in this material [10]. Isotopically anomalous Mg was found in CAIs [11][12]. There were samples with both excesses and deficiencies in ${ }^{26} \mathrm{Mg}$ (a few per mil) in samples with 50 per mil enhancements in ${ }^{16} \mathrm{O} .{ }^{26} \mathrm{Al}$ was then found to have been present in CAIs through a clear correlation of excess ${ }^{26} \mathrm{Mg}\left({ }^{26} \mathrm{Mg}^{*}\right)$ with ${ }^{27} \mathrm{Al}$ in different minerals with widely varying $\mathrm{Mg} / \mathrm{Al}$ ratios [13] [14]. Early suggestions by Urey [15] and Urey \& Donn [16] indicated that the nuclide

${ }^{26} \mathrm{Al}$, which had just then been discovered in a cyclotron target [17], was the 
only reasonable source for the early heating and melting of planetesimals. The authors noted that: "If the problem of producing sufficient quantities of ${ }^{26} \mathrm{Al}$ can be solved, this nuclide should be of considerable value in chemistry, metallurgy, and related fields" [17]. As pointed out by Urey [15], the heating and melting of small planetesimals required a short-lived radioactivity of a major element as the heat source, since there would not be sufficient gravitational energy to melt them.

A few years before the fall of the Allende meteorite, the presence of a relatively long-lived species, possibly ${ }^{244} \mathrm{Pu}(\bar{\tau}=115 \mathrm{Myr})$, had been inferred from excesses of neutron-rich Xe isotopes in planetary differentiates [18]. Subsequent work showed that the enrichment in unshielded Xe isotopes was directly associated with excess fission tracks in meteoritic minerals rich in U, Th, and REE [19] [20] [21] [22] and required the in situ fission of a transuranic nuclide (possibly ${ }^{244} \mathrm{Pu}$ ). The Xe isotopic composition in these meteorite samples showed very large excesses in ${ }^{131,132,134,136} \mathrm{Xe}$. The pattern was identical to that resulting from ${ }^{244} \mathrm{Pu}$ spontaneous fission subsequently established in the laboratory by Alexander et al. [23]. ${ }^{244} \mathrm{Pu}$-fission Xe was also found in the CAIs from Allende [7]. The hint of excesses of ${ }^{142} \mathrm{Nd}$ by Lugmair \& Marti [24] pointed to the possible presence of ${ }^{146} \mathrm{Sm}(\bar{\tau}=148 \mathrm{Myr})$, a $p$-process isotope that $\alpha$-decays to ${ }^{142} \mathrm{Nd}$ with cosmochronologic implications [25]. Excesses of ${ }^{142} \mathrm{Nd}$ were found to be widespread, both in ESS materials and in planetary differentiates (PD), and to be well correlated with Sm in planetary differentiates [24] [26] [27]; see also [28]. Evidence for the $p$ nuclide ${ }^{92} \mathrm{Nb}(\bar{\tau}=52 \mathrm{Myr})$ was found by Harper [29]. The presence of ${ }^{107} \mathrm{Pd}(\bar{\tau}=9.4 \mathrm{Myr})$ was established by Kelly \& Wasserburg [30]; this nuclide, which is produced by both $r$ and $s$ processes, was found to have been present in a large number of iron meteorites representing metal segregation in protoplanets or planetesimals [31] [32] [33]. The results on ${ }^{107} \mathrm{Pd}$ showed that planetary cores formed very early in the history of the solar system. The discovery of tungsten isotopic anomalies (deficiencies) in ${ }^{182} \mathrm{~W}$ in iron meteorites and their correlation with $\mathrm{Hf}$ in chondritic meteorites demonstrated the presence of ${ }^{182} \mathrm{Hf}(\bar{\tau}=13 \mathrm{Myr})$ in early planets at the time of core formation [34] [35] [36][37].

Both the possible presence of ${ }^{53} \mathrm{Mn}(\bar{\tau}=5.3 \mathrm{Myr})$ in CAIs, with a correlation of ${ }^{53} \mathrm{Cr}^{*}$ with Mn, suggested by Birck and Allègre [38] [39], and the subsequent clear demonstration of abundant ${ }^{53} \mathrm{Mn}$ in planetary differentiates [39][40][41], stimulated new attention to the nucleosynthetic processes in supernovae (SNe II or SNIa) and in spallation processes. The hints of ${ }^{60} \mathrm{Fe} \quad(\bar{\tau}=2.2 \mathrm{Myr})$ discovered by Shukolyukov \& Lugmair [42] [43] and the subsequent recent demonstration of ${ }^{60} \mathrm{Ni}$ excesses correlated with $\mathrm{Fe} / \mathrm{Ni}$ in chondrites, require ${ }^{60} \mathrm{Fe}$ to be present at rather high abundances in the early solar system [44] [45] [46]. This nucleus has connections to both supernovae and asymptotic giant branch (AGB) sources, but not to spallation reactions. There is the possibility of ${ }^{205} \mathrm{Tl}$ excesses that would indicate the presence of ${ }^{205} \mathrm{~Pb}(\bar{\tau}=22$ 
Myr, a shielded nucleus), which, like ${ }^{204} \mathrm{~Pb}$, is certainly from the $s$-process [47].

The discovery of the very short-lived ${ }^{41} \mathrm{Ca} \quad(\bar{\tau}=0.15 \mathrm{Myr})$ by Srinivasan, Ulyanov \& Goswami [48] and Srinivasan et al. [49] has very important ramifications. Although abundantly produced in AGB stars, its short time scale may present a problem. At the low abundance observed, it might also be produced by proton bombardment, but it is correlated with ${ }^{26} \mathrm{Al}$. The important discovery of ${ }^{10} \mathrm{Be}(\bar{\tau}=2.3 \mathrm{Myr})$ in the early solar system by McKeegan, Chaussidon and Robert [50] has demonstrated the presence of a nuclide that is not a product of stellar nucleosynthesis, but requires proton bombardment of small solids.

In addition, Allende provided a whole host of small, but clearly measurable, isotopic anomalies in many elements (a Pandora's box of the nuclides), which demonstrated that incompletely mixed material from different presolar sources was preserved in macroscopic samples of solar system processed material in meteorites. These "isotopic anomalies" and proposals of various nucleosynthetic mechanisms caused lots of excitement. They also resulted in some difficulty in unraveling the presence and abundances of some short-lived nuclei in CAIs, as there was not always a clear base line of initial isotopic composition in some of these samples.

A prescient study by Black [51] led to the remarkable result that almost pure ${ }^{22} \mathrm{Ne}$ was present in chondrites. Black attributed this to the preservation of presolar grains from Red Giant Branch (RGB) stars. This led to a long and difficult chase to find such grains. The result was in the major discovery of individual unprocessed refractory presolar dust grains in chondritic meteorites [52] [53]. These grains provide direct evidence on the nature of potential contributors to the solar nebula and of aspects of stellar nucleosynthetic processes that were previously not available (see Section 2). In particular, s-process abundance patterns can now be compared with the observed abundances in circumstellar dust grains. It is now known that dust grains from diverse AGB stars are a substantial or major source of the condensed matter that was incorporated into the early solar system. Reviews of astronomical observations on dust are given by Draine [54] [55] [56].

In this report we present a review of the short-lived radioactivities $\left(10^{9}>\right.$ $\left.\bar{\tau}>10^{5} \mathrm{yr}\right)$ that were present in the early solar system and the potential stellar sources of these nuclides. This panoply of short- to intermediate-lifetime nuclei provides the direct connection to nuclear astrophysical processes and a host of exciting and confusing possibilities in search of real explanations. For an extensive review of the experimental data on the early solar system abundances of short-lived nuclei, we refer the reader to McKeegan \& Davis [57]. In particular, we will focus on the possible addition of fresh stellar debris into the protosolar nebula from a single source, with an emphasis on possible 
AGB contributions. Some intermediate and longer lived nuclei will be shown to come most plausibly from long-term galactic nucleosynthesis. We will also review some of the characteristics of pre-solar circumstellar condensates that are preserved in meteorites, relating to the production of ${ }^{26} \mathrm{Al}$ in stellar models. A critical matter will be the nature of nucleosynthetic yields of potential sources. A recent review of the short-lived nuclei by Busso et al. [58] will be used as the source for the present report. Substantial new results will be presented concerning the characteristics of AGB stars of a range in masses and with specific consideration of the results of cool bottom processing (see Section 4).

\section{Circumstellar Dust Grains}

A wide variety of grains has been discovered in residues from chondrites that showed gross variations of the isotopic ratios of major elements not related to radioactive decay. These grains demonstrated that presolar dust from a wide variety of stellar sources was the net material from which the solar system formed (see reviews by Anders \& Zinner on this discovery [52] [53]). A recent review of "Astrophysics of Stardust" [59] is a useful guide here.

Some grains contained clear evidence of ${ }^{26} \mathrm{Al}$ when they formed [60]. Some small fraction of these grains were presumably the carriers that made up the solar inventory of the short-lived radioactive nuclides. However, most of the extrasolar grains were sufficiently old that the radioactive nuclei in them had already decayed before the solar system formed. Until recently, only refractory grains had been recovered. There is extensive recycling of the grains within the interstellar medium (ISM) and in diverse stars; only a limited sampling is available. Much earlier generations of stellar debris are, of course, involved in making up the solar inventory; this includes gas (atoms, molecules, and ions) and stellar dust that has been cycled-recycled by a variety of processes in the ISM (see Draine [54] [55], [56]). The gas is depleted in "non-volatile" elements which are mostly resident in the dust phase. The phases responsible for carrying the elemental budget in the ESS involves all of the above components. Some "live" nuclei present in the ESS, such as ${ }^{129} \mathrm{I}$, may be present in the gas phase and others (like ${ }^{26} \mathrm{Al}$ or ${ }^{182} \mathrm{Hf}$ ) are certainly in some dust particles.

The ${ }^{12} \mathrm{C} /{ }^{13} \mathrm{C}$ and ${ }^{14} \mathrm{~N} /{ }^{15} \mathrm{~N}$ isotopic compositions of circumstellar SiC grains recovered from meteorites are shown in Fig. 1. A histogram showing the frequency of occurrence of a given ${ }^{12} \mathrm{C} /{ }^{13} \mathrm{C}$ ratio is shown at the base of the figure. It is evident that the predominant population of grains lies in the range of $40<{ }^{12} \mathrm{C} /{ }^{13} \mathrm{C}<100$. These are the so-called "mainstream" grains and represent the value expected for AGB stars. There is a small population with very low ${ }^{12} \mathrm{C} /{ }^{13} \mathrm{C}$ that remains an unresolved problem (see [61]). The ${ }^{14} \mathrm{~N} /{ }^{15} \mathrm{~N}$ 
ratios require special attention (see Section 4 ). The ${ }^{26} \mathrm{Al} /{ }^{27} \mathrm{Al}$ abundances in some of these grains are shown in Fig. 2; the observed range is $10^{-5}<{ }^{26} \mathrm{Al} /{ }^{27} \mathrm{Al}$ $<2 \times 10^{-2}$.

Isotopic analyses of individual $\mathrm{SiC}$ grains for heavy elements have recently become possible. This was primarily a result of major instrumental development by M. Pellin and his colleagues at Argonne National Laboratory (cf. [62] [63] [64]); similar measurements have now become feasible with different techniques in other laboratories [65]. The results on a number of $\mathrm{SiC}$ grains from the "mainstream" population show clear and definitive enrichments in $s$ process nuclei and deficiencies in $r$ and $p$ nuclei (see example in Fig. 3). There is even evidence of ${ }^{99} \mathrm{Tc}$ from $\mathrm{SiC}$ grains [66]. The presence of these enrichments of $s$-process nuclei greatly strengthens the assignment of this population of carbide grains to AGB sources with significant neutron exposures. These observations are in full accord with the direct astronomical observations of enrichment of $s$-process "elements" in AGB stars.

The discovery of circumstellar oxide grains in meteorites [67] [68] [69] [70] and the important and extensive results by Nittler et al. [70][71][72] revealed that most of these grains also appear to come from AGB sources. Fig. 4 shows a compilation of oxygen isotopic data from individual refractory oxide grains (corundum, hibonite, spinel). It can be seen that the preponderance of the grains show ${ }^{18} \mathrm{O} /{ }^{16} \mathrm{O}<\left({ }^{18} \mathrm{O} /{ }^{16} \mathrm{O}\right)_{\odot}$ and ${ }^{17} \mathrm{O} /{ }^{16} \mathrm{O}>\left({ }^{17} \mathrm{O} /{ }^{16} \mathrm{O}\right)_{\odot}$. This is in general accord with the effects expected from first dredge-up in Red Giant stars (see Dearborn [73]). However, observations on the grains show overproduction of ${ }^{17} \mathrm{O}$ and much more extensive destruction of ${ }^{18} \mathrm{O}$ that is far outside the range to be expected for standard RGB or AGB models [71] [74].

A compilation of the available ${ }^{26} \mathrm{Al}$ data is shown in Fig. 5. Again, as is the case for carbide grains, the data show an enormous range in ${ }^{26} \mathrm{Al} /{ }^{27} \mathrm{Al}$, up to a few times $10^{-2}$. Clear evidence of ${ }^{41} \mathrm{~K}^{*}$ is found in some circumstellar oxide grains from the decay of ${ }^{41} \mathrm{Ca}$. Measurement of potassium isotopes in Ca-rich oxide grains (hibonites) show ${ }^{41} \mathrm{~K}$ correlated with $\mathrm{Ca} / \mathrm{K}$. The inferred ${ }^{41} \mathrm{Ca} /{ }^{40} \mathrm{Ca}$ for some grains is in excellent agreement with the AGB model values, although other grains show much lower values that still require explanation [74] [75].

A most exciting discovery has been made of silicate grains from circumstellar sources by Nguyen \& Zinner [76], by Nguyen, Zinner \& Stroud [77], and by Hoppe, Mostefaoui \& Stephan [78], all in the Acfer meteorite, and also by Mostefaoui, Marhas \& Hoppe [79] in Bishunpur. Such presolar grains were first found in interplanetary dust particles by Messenger et al. [80]. This new generation of measurements was made possible by major technical developments by George Slodzian. This permits analysis of sub-micron grains. These circumstellar silicate grains (long sought for) appear to be far higher in abundance than other presolar grains. In general, they appear to have their origin 
in AGB stars. This strongly emphasizes the points laid out above about the importance of AGB contributions to the ESS. These observations open up an exciting area of research.

The results outlined above attest to the major contribution of carbide, carbon, oxide, and now silicate grains from diverse AGB stars to the initial solar system chemical and isotopic abundances. They do not, however, identify any source that provided short-lived nuclei to the ESS. In addition to the preponderance of grains attributable to AGB stars, there are a relatively rare subset of some of the $\mathrm{SiC}$ and graphite grains and a few oxide grains that are probably (sometimes certainly) from SNe sources [74] [75] [81] [82]. These account for a fraction of about $10^{-2}$ of the grains observed. If the available sampling is representative, then this must limit the level of late contributions from a supernova. The individual grains give us a view of possible contributing sources to the short-lived nuclei, but they do not define these sources or the amount contributed by them. Most meteoritic material has been chemically and thermally processed in the solar system, so that the individual components are typically homogenized.

\section{Review of Nucleosynthesis in AGB Stars}

In considering the matter of short-lived nuclei, the basic observational data are the abundances of the relevant radioactive nuclei at some arbitrary "initial" time. As our main focus will be possible AGB sources, we will first give a short review of AGB nucleosynthesis considering the standard model. Then we will present a more extensive review of cool bottom processing (which has caused significant changes in the treatment of ${ }^{26} \mathrm{Al}$ ) and of AGB contributions.

\subsection{Standard Models}

A thorough review of the status of stellar models is presented by Straniero et al. [83] in this volume. For low mass stars, after exhaustion of the $\mathrm{H}$ in the core, energy is only produced by a H-burning shell, leaving a He core. The star then develops a fully convective envelope structure and ascends the H-R diagram to the red giant branch (RGB), where surface convection first enters previously radiative layers and mixes material that had previously experienced there proton captures to the photosphere (first dredge-up). Observations show that the model predictions for the first dredge-up are in rough agreement with the spectroscopic abundances of $\mathrm{C}$ and $\mathrm{O}$ isotopes in RGB stars of masses above $\simeq 2.5 M_{\odot}$ but not for lower masses (see e.g. [84]). At the end of the RGB stage, He is ignited and a C-O core evolves. When the 
He at the center is finally exhausted, energy is produced by H-shell burning, with regular short interruptions from thermal instabilities at the top of the C-O core. In each interruption, the He shell burns for a short time. Thus, the $\mathrm{H}$ shell and the He shell burn alternately over a very narrow region of mass in the star. The He-shell ignition is followed by the convective penetration of the envelope through the then inactive H-burning shell (third dredge-up or TDU). This stage is referred to as the thermally pulsing AGB phase (TPAGB: see [85]). The third dredge-up mixes processed material from the $\mathrm{H}-\mathrm{He}$ zone interface region into the convective envelope and thus the photosphere, where the changes in composition can be observed. This involves addition of major reaction products made by proton reactions (e.g., ${ }^{13} \mathrm{C},{ }^{14} \mathrm{~N}$ ) as well as by $\alpha$-capture reactions and neutron captures (the $s$-process nuclei). TDU does not change the $\mathrm{O}$ isotopic composition.

In the nineties, improvements in the input physics of stellar models (equation of state, opacities) and the advent of cheap and fast computers allowed the third dredge-up to be self-consistently modeled down to low masses, thus yielding the first theoretical interpretation of low-luminosity C stars [86] [87]. It is this class of stellar models (see [83], this volume, and references therein) that provides the temperature, density, electron density, and effective chemical composition in each zone over the stellar lifetimes. The input parameters for such models are: the initial chemical composition of the star; its initial mass; and the rate of loss of the envelope. In order to save computer time, detailed nucleosynthesis calculations for neutron capture nuclei and other minor species are, even now, often omitted in the complete stellar models as they do not affect the energy budget and hence do not affect the stellar structure. In these cases, the outputs of stellar models are then used as a basis for post-process computations in which large networks involving thousands of reactions can be used. However, the major stellar codes (e.g. the Australian MSSSP, or the Italian FRANEC) can now be run in individual control cases using the whole reaction set [88] [89], thereby providing the nodes of a grid. Stellar parameters for other cases can then be deduced by suitable interpolations [89].

In AGB stars two major neutron sources are at play: the ${ }^{22} \mathrm{Ne}(\alpha, n){ }^{25} \mathrm{Mg}$ reaction [90] and the ${ }^{13} \mathrm{C}(\alpha, n){ }^{16} \mathrm{O}$ reaction [91] [92] [93]. Both reactions take place in the He shell, but only the first is a direct and intrinsic consequence of the stellar evolution. The neutron densities produced from ${ }^{22} \mathrm{Ne}$ burning reflect the temperature and thus the stellar mass. The reaction products of neutron capture from this source do not depend on any parameters other than the initial stellar abundances and the basic stellar model. The second reaction producing neutrons is ${ }^{13} \mathrm{C}(\alpha, n){ }^{16} \mathrm{O}$ and requires that matter rich in ${ }^{12} \mathrm{C}$ (from the He shell) must react with protons to produce ${ }^{13} \mathrm{C}$ in a layer of the $\mathrm{He}-\mathrm{H}$ intershell called the ${ }^{13} C$ pocket. This mixing scenario requires some non-convective process to bring protons into the intershell region where there is abundant ${ }^{12} \mathrm{C}$ [94]. A very good representation of the solar system "main $s$-component" can be ob- 
tained from a galactic chemical evolution model that uses the outputs of AGB stars of different generations, including products from both neutron sources [95] [96], the dominant contributor being the ${ }^{13} \mathrm{C}(\alpha, n)^{16} \mathrm{O}$ source. However, the strength of this source must be treated as a free input parameter in the absence of any self-consistent model for the required proton mixing.

The presentation here with regard to possible AGB sources to account for some short-lived nuclei in the early solar system is an advanced treatment of the earlier approach [97]. In that report, we used more approximate stellar models developed by other workers and computed nucleosynthesis in AGB stars with a post-processing calculation based on a schematic representation of the thermal pulses. This involved two key parameters: 1) The neutron exposure producing $s$-process nuclei; and 2) the mass of the star, which controls the temperature of the H-burning shell for ${ }^{26} \mathrm{Al}$ production. These parameters then established a relationship between the mass of the possible stellar source, the net neutron exposure, and the amount of ejected AGB envelope that must mix with the ambient ISM to provide the inventory of radioactive nuclei in the solar nebula. The input parameters used were the observed ESS ${ }^{107} \mathrm{Pd}$ abundance as a monitor of the neutron exposure, and ${ }^{26} \mathrm{Al}$ as a monitor of the proton captures on ${ }^{25} \mathrm{Mg}$ in the H-burning shell (and of its severe destruction by neutron captures in the He shell, during thermal pulses). Using these parameters, it was possible to obtain a self-consistent abundance pattern for several other nuclides and to predict the abundances of radioactive nuclei that had not been observed. The ${ }^{26} \mathrm{Al}$ produced in the standard model gave ${ }^{26} \mathrm{Al} /{ }^{27} \mathrm{Al}$ $\sim(1-3) \times 10^{-3}$. This model was certainly not sufficient to provide the high ${ }^{26} \mathrm{Al} /{ }^{27} \mathrm{Al}$ observed in some circumstellar dust grains attributed to AGB stars (see Figs. 2 and 5).

Recognition that additional proton processing of envelope material just above the H-burning shell was necessary resulted from the laboratory observations of oxygen isotopes in circumstellar dust grains found in meteorites and from astronomical observations of ${ }^{12} \mathrm{C} /{ }^{13} \mathrm{C}$ in low-mass RGB stars. This additional mixing and reaction mechanism fundamentally alters the problem for AGB stars and was discussed by Busso, Gallino \& Wasserburg [84] in their review of short-lived nuclei. The result is that ${ }^{26} \mathrm{Al}$ in AGB stars may effectively be governed by reactions just above the $\mathrm{H}$ burning shell due to some transport in a phenomenon called "extra mixing" or Cool Bottom Processing (CBP). This process must also occur in low mass stars on the Red Giant Branch [98] [99], but would not produce much ${ }^{26} \mathrm{Al}$ there due to the lower temperature of the $\mathrm{H}$ shell. The amount of this processing on the AGB is not a priori known and is a free parameter. It is therefore not possible to use the ${ }^{26} \mathrm{Al}$ abundance as the basis for estimating the AGB input. Thus the effort to provide a self-consistent model is left open, since there are no other radionuclides with lifetimes of $\sim 10^{6}$ yr that are only produced by normal AGB evolution and whose early solar system abundance has been established at a reasonably precise value. Because 
of the importance of CBP in AGB stars, we provide an extensive summary of the effects of this mechanism on both radioactive and stable nuclei.

\section{Cool Bottom Processing}

Convection and mixing inside stars cannot be treated from first principles in one-dimensional models. Instead, these phenomena are treated through approximations, applying stability criteria to determine whether energy transport in a given layer is radiative or convective. Convective regions are described in the mixing length formalism, and their compositions homogenize rapidly. Radiative regions are assumed to be free of matter circulation. However, there is clear observational evidence for partial mixing in the radiative regions, so mechanisms must be available to drive circulation there as well (e.g., Herwig [100] and references therein). A prime example is weak activation of the CN cycle attributed to material circulating below the convective envelope of stars on the red giant branch, as mentioned above and in [98] [100] [101] [102]. Further evidence may be found in the observation of an extremely metal poor star with $[\mathrm{Fe} / \mathrm{H}]=-2.72$ by Lucatello et al. [103] (here $\left.[\mathrm{Fe} / \mathrm{H}]=\log (\mathrm{Fe} / \mathrm{H})_{\text {star }}-\log (\mathrm{Fe} / \mathrm{H})_{\odot}\right)$. These workers found that $[\mathrm{Pb} / \mathrm{Fe}]=3.3$, $[\mathrm{C} / \mathrm{Fe}]=2.6$ and $\left({ }^{12} \mathrm{C} /{ }^{13} \mathrm{C}\right)=6$. This carbon isotopic ratio could not be attained by standard AGB models as they would yield $\left({ }^{12} \mathrm{C} /{ }^{13} \mathrm{C}\right) \gtrsim 4 \times 10^{4}$ and thus these results are a further strong indication of $\mathrm{CBP}$.

In models of cool bottom processing, it is assumed that slow circulation begins at the bottom of the star's convective envelope and carries envelope material down to layers dense and hot enough for some nuclear processing to occur but not far enough to result in significant energy generation. A return flow maintains the stellar structure in steady state, returning processed material to the fully convective envelope.

Because the physical mechanism driving CBP is not known, its action inside any given star is described in the models by two parameters, a depth of mixing and a rate of mixing, specified in the following ways: 1) The maximum depth of mixing may be specified as maximum temperature seen by the processed material $\left(T_{P}\right)[104][61][105]$, or as the fraction of the mass $(\delta M)$ of the radiative region above the hydrogen shell where the slow mixing takes place [106] [107] [108]; 2) The rate of mixing is specified either by the mass circulated per year $(M)$ [104] [61] [105] or by a parametric diffusion coefficient $\left(D_{\text {mix }}\right)$ [106] [107] [108]. The two sets of parameters (circulation rate and maximum temperature versus diffusion coefficient and mass region) are completely equivalent and indistinguishable as far as observable effects are concerned, and relations between the two representations are discussed in Nollett et al. [61]. Here, we use the parameter set $\left(T_{P} / T_{\mathrm{H}}, \dot{M}\right)$ as in Messenger [105], where $T_{\mathrm{H}}$ 
is the temperature of the hydrogen-burning shell.

Computations of cool bottom processing may be performed by post-processing, with the advantage that rapid exploration of the CBP parameter space is then possible. The mixing should move faster than the rate of advance of the hydrogen shell, so as not to be overtaken, and the rate of energy release that results from this mixing should be very much less than the luminosity of the hydrogen shell. For the stars of interest, this corresponds to $\dot{M}>10^{-7} M_{\odot} /$ yr and $\log \left(T_{P} / T_{\mathrm{H}}\right) \leq-0.1$ Moreover, the rate of mass turnover in the cool bottom processing region should be much lower than the rate of mass turnover in the envelope above, corresponding to $\dot{M} \lesssim 10^{-4} M_{\odot} /$ yr. We assume that CBP operates throughout the hydrogen shell-burning intervals between thermal pulses.

Surface compositions produced by cool bottom processing result from the interplay of three rules [104] [61]: 1) the amount of nuclear processing increases with the depth of mixing $\left(T_{P}\right) ; 2$ ) the amount of nuclear processing in the circulation stream decreases with $\dot{M}$ because a higher circulation rate results in less time spent at high temperature by a fluid element; 3) the amount of processed material that ends up in the stellar envelope increases with $\dot{M}$.

We previously explored the consequences of these rules, both analytically and in numerical post-processing models, for the case of the TP-AGB phase of a star with a $1.5 M_{\odot}$ initial mass and initial solar system composition [61]. In subsequent calculations summarized here, we found that essentially the same consequences arise in stars with initial masses of 2 and $3 M_{\odot}$, and also at lower metallicities. At masses beyond $3 M_{\odot}, \mathrm{CBP}$ is not possible, because the hydrogen burning shell reaches temperatures of $T_{\mathrm{H}}>10^{8} \mathrm{~K}$ and the bottom layers of the convective envelope are also hot enough for proton captures to occur. These conditions are called hot bottom burning (HBB), and they are explored thoroughly by other authors (cf. [109] [110] [111]).

\subsection{Production of ${ }^{26} \mathrm{Al}$}

The rate of ${ }^{26} \mathrm{Al}$ enrichment of the stellar envelope does not depend on $\dot{M}$, but depends only on $T_{P}$, through the rate for the ${ }^{25} \mathrm{Mg}(p, \gamma)^{26} \mathrm{Al}$ process. The product of the rate of ${ }^{26} \mathrm{Al}$ transfer to the envelope with the TP-AGB lifetime determines the maximum amount of ${ }^{26} \mathrm{Al}$ that can be made. For several different stellar models in the initial mass range $1.5-3.0 M_{\odot}$, the envelope ${ }^{26} \mathrm{Al}$ enrichment rate shows a near-regularity when plotted against $\log \left(T_{P} / T_{\mathrm{H}}\right)$ as shown in the insert of Fig. 2. This regularity holds rather generally, but its normalization changes with time during the evolution of a single star. It is a robust result that for a given stellar model, the amount of ${ }^{26} \mathrm{Al}$ that can

be produced during the TP-AGB phase varies from the level ${ }^{26} \mathrm{Al} /{ }^{27} \mathrm{Al} \simeq$ a 
few times $10^{-3}$ provided by third dredge-up to a few times $10^{-2}$. Thus CBP can adequately explain the population of ${ }^{26} \mathrm{Al} /{ }^{27} \mathrm{Al}$ found in both carbide and oxide grains (see Figs. 2 and 5). Note that without CBP, a large fraction of the ${ }^{26} \mathrm{Al}$ that is produced is subsequently destroyed in the He shell (cf. [97]). The lowest values in the grains must then result either from stars without CBP and with less efficient production in the H-burning shell, or from more effective destruction of ${ }^{26} \mathrm{Al}$ in the He shell (without CBP) than predicted in the standard models.

\subsection{Destruction of ${ }^{18} \mathrm{O}$}

The case of ${ }^{18} \mathrm{O}$ brings out the third rule listed above: the amount of processed material brought to the envelope increases with $\dot{M}$. Even at $\log T_{P} / T_{\mathrm{H}} \simeq-0.2$, essentially all of the ${ }^{18} \mathrm{O}$ in the $\mathrm{CBP}$ stream is destroyed by the reaction ${ }^{18} \mathrm{O}(p, \alpha){ }^{15} \mathrm{~N}$. Thus the material circulates down and returns to the envelope, and the ${ }^{18} \mathrm{O}$ remaining in the envelope is diluted with ${ }^{18} \mathrm{O}$-free material. The envelope ${ }^{18} \mathrm{O} /{ }^{16} \mathrm{O}$ declines exponentially with a time constant $M_{E} / \dot{M}$, where $M_{E}$ is the mass of the stellar envelope. For the maximum $\dot{M}$ considered $\left(10^{-4} M_{\odot} / \mathrm{yr}\right)$ and an envelope mass of $0.7 M_{\odot}$, this comes to $M_{E} / \dot{M}=1.4 \times 10^{4} \mathrm{yr}$, considerably less than the total time spent in the TPAGB phase. Thorough depletion of ${ }^{18} \mathrm{O}$ is therefore a sign of $\mathrm{CBP}$, and it sets in at lower $T_{P}$ than needed for significant ${ }^{26} \mathrm{Al}$ production.

It was suggested by Nittler at al. [71] and Choi, Wasserburg, \& Huss [74] that ${ }^{26} \mathrm{Al} /{ }^{27} \mathrm{Al}$ and ${ }^{18} \mathrm{O} /{ }^{16} \mathrm{O}$ would be correlated. However, each of these ratios is sensitive to a different $\mathrm{CBP}$ parameter. With $\mathrm{CBP}$, the ${ }^{26} \mathrm{Al}$ production depends almost entirely on $T_{P}$. The ${ }^{18} \mathrm{O}$ depletion depends only on $\dot{M}$ with a relatively low temperature threshold $\left(\log T_{P} / T_{\mathrm{H}} \geq-0.2\right)$. See Fig. 6 of [61]. It is thus possible to obtain destruction of ${ }^{18} \mathrm{O}$ with or without significant ${ }^{26} \mathrm{Al}$ production and substantial ${ }^{26} \mathrm{Al}$ production with or without ${ }^{18} \mathrm{O}$ destruction; however, conditions on oxide versus carbide production discussed below place further constraints on which compositions are possible in a given type of grain.

\subsection{Equilibration of ${ }^{17} \mathrm{O} /{ }^{16} \mathrm{O}$}

Above a relatively low threshold in $T_{P},{ }^{17} \mathrm{O} /{ }^{16} \mathrm{O}$ in the circulating material reaches an equilibrium by balancing rates for reactions ${ }^{16} \mathrm{O}(p, \gamma){ }^{17} \mathrm{~F}\left(\beta^{+} \nu\right){ }^{17} \mathrm{O}$ and ${ }^{17} \mathrm{O}(p, \alpha){ }^{14} \mathrm{~N}$. Given the presently recommended reaction rates [112], the equilibrium ratio has a broad minimum in exactly the temperature range of interest for AGB stars, so that for all cases CBP should produce ${ }^{17} \mathrm{O} /{ }^{16} \mathrm{O}$ $\cong 0.0011$. (This value may be slightly changed because of a recent revision 
of the rates for proton capture on ${ }^{17} \mathrm{O}$ [113].) Thus, when plotted on a threeisotope plot for oxygen isotopes, abundances in the stellar envelope after some CBP will lie on a mixing line connecting the composition at the start of the AGB phase with a composition that has the equilibrium value of ${ }^{17} \mathrm{O} /{ }^{16} \mathrm{O}$. One then expects to find grains heavily depleted in ${ }^{18} \mathrm{O}$ that simultaneously have ${ }^{17} \mathrm{O} /{ }^{16} \mathrm{O}=0.0011 \pm 0.0003$. Oxide grains fitting this description have been found in the data of several groups of authors (see Fig. 4).

\subsection{Carbon and Nitrogen}

The $\mathrm{C}$ and $\mathrm{N}$ isotopes are also affected by CBP. However, this is more complicated because third dredge-up contributes significant amounts of carbon to the envelope. The effects of CBP on ${ }^{12} \mathrm{C} /{ }^{13} \mathrm{C}$ and ${ }^{14} \mathrm{~N} /{ }^{15} \mathrm{~N}$ mostly consist of processing the large amounts of ${ }^{12} \mathrm{C}$ added to the envelope by third dredge-up. In the case of ${ }^{15} \mathrm{~N}$ the effect is simple: it has a very effective destruction mechanism, ${ }^{15} \mathrm{~N}(p, \alpha){ }^{12} \mathrm{C}$, that makes the effects on ${ }^{15} \mathrm{~N}$ exactly analogous to those on ${ }^{18} \mathrm{O}$. The effects on the carbon isotopes and on ${ }^{14} \mathrm{~N}$ depend in a somewhat complicated way on $T_{P}$ and $\dot{M}$, following the three rules as outlined above. In brief, $\mathrm{CBP}$ converts ${ }^{12} \mathrm{C}$ to ${ }^{13} \mathrm{C}$, and then to ${ }^{14} \mathrm{~N}$.

In the absence of CBP, the envelope abundance of ${ }^{12} \mathrm{C}$ increases with time, in a discrete jump following each thermal pulse. Eventually, if enough ${ }^{12} \mathrm{C}$ is brought to the stellar envelope, the surface composition attains $\mathrm{C} / \mathrm{O}>1$ and the consequent changes in chemistry follow. At moderate $T_{P}, \mathrm{CBP}$ lowers the ratio ${ }^{12} \mathrm{C} /{ }^{13} \mathrm{C}$ in the stellar envelope by converting some of the newly-formed

${ }^{12} \mathrm{C}$ into ${ }^{13} \mathrm{C}$. For some values of $\dot{M}$, this can bring the envelope abundance ratio down to ${ }^{12} \mathrm{C} /{ }^{13} \mathrm{C} \simeq 5$ while leaving $\left({ }^{13} \mathrm{C}+{ }^{12} \mathrm{C}\right) / \mathrm{O}$ relatively unchanged. If, however, $T_{P}$ is a little higher (or $\dot{M}$ a little lower), then the ${ }^{13} \mathrm{C}$ is rapidly converted into ${ }^{14} \mathrm{~N}$. At low values of $\dot{M}$, the ratio ${ }^{12} \mathrm{C} /{ }^{13} \mathrm{C}$ approaches $\sim 4$ from the $\mathrm{CN}$-cycle equilibrium. However, the material returning to the envelope when $T_{P}$ is high is depleted in carbon of both isotopes so that its main effect is to reduce $\mathrm{C} / \mathrm{O}$ without necessarily having a large effect on ${ }^{12} \mathrm{C} /{ }^{13} \mathrm{C}$. For high enough $\dot{M}$ and $T_{P}$, a large fraction of the ${ }^{12} \mathrm{C}$ brought up by third dredge-up can be converted into ${ }^{14} \mathrm{~N}$, delaying or preventing the formation of a carbon star. The results of CBP calculations show a well-defined relationship between ${ }^{12} \mathrm{C} /{ }^{13} \mathrm{C}$ and $\mathrm{C} / \mathrm{O}$ as the $\mathrm{CBP}$ parameters vary. Since ${ }^{26} \mathrm{Al}$ is effectively a thermometer and $\mathrm{C} / \mathrm{O}$ a measure of $\dot{M}$ and $T_{P}$, the condition of a star is rather well determined by ${ }^{26} \mathrm{Al},{ }^{12} \mathrm{C} /{ }^{13} \mathrm{C}$, and $\mathrm{C} / \mathrm{O}$.

Stars whose evolution into a carbon star has been prevented will have large $\mathrm{N} / \mathrm{O}$ abundance ratios, because of the conversion of newly-produced carbon into nitrogen. Such nitrogen-rich final abundances (observed, perhaps, in planetary nebulae) can in principle constitute evidence for CBP. However, this is 
also a fairly generic result of hot bottom burning, so that information about the stellar mass is needed to make a clear assignment to CBP. An apparent case of a planetary nebula with high enough $\mathrm{N} / \mathrm{O}$ and low enough progenitor mass for CBP to be important has been reported [114].

Without CBP, the production and dredge-up of $\mathrm{C}$ eventually make an envelope with $\mathrm{C} / \mathrm{O}>1$. This should result in carbide grains forming, while extreme destruction of $\mathrm{C}$ by $\mathrm{CBP}$ will give $\mathrm{C} / \mathrm{O}<1$ and should result in oxide grains forming [115] [116]. We expect that if CBP were active in the stars that formed presolar grains, abundances in the carbide grains should reflect the parts of the CBP parameter space consistent with $\mathrm{C} / \mathrm{O}>1$, while those in oxide grains should reflect $\mathrm{CBP}$ parameters that preserve $\mathrm{C} / \mathrm{O}<1$. This is broadly true, though there are some discrepancies (see Fig. 4). In particular, it is difficult to account for simultaneous high ${ }^{26} \mathrm{Al} /{ }^{27} \mathrm{Al}$ and high ${ }^{18} \mathrm{O} /{ }^{16} \mathrm{O}$ in an oxide grain, because high ${ }^{26} \mathrm{Al} /{ }^{27} \mathrm{Al}$ indicates $\mathrm{CBP}$ operating for a long time, while high ${ }^{18} \mathrm{O} /{ }^{16} \mathrm{O}$ implies low $\dot{M}$ and thus $\mathrm{C} / \mathrm{O}>1$ at late times. Thus the red point and some of the green points in Figure 5 with ${ }^{18} \mathrm{O} /{ }^{16} \mathrm{O}>0.001$ are examples of discrepancies (cf. Figs. 7b and 10 of [61]).

The nitrogen isotopic ratios observed in the circumstellar grains are difficult to understand in terms of AGB evolution, even without CBP. They should have high values of ${ }^{14} \mathrm{~N} /{ }^{15} \mathrm{~N}$ because first dredge-up brings a great deal of ${ }^{15} \mathrm{~N}$-depleted and ${ }^{14} \mathrm{~N}$-enriched material to the stellar envelope. Previous calculations starting with solar-system initial composition have found that the increase in ${ }^{14} \mathrm{~N} /{ }^{15} \mathrm{~N}$ in the envelope at first dredge-up is roughly a factor of five. The mainstream grains show ${ }^{14} \mathrm{~N} /{ }^{15} \mathrm{~N}$ extending all the way down to the solar value (see Fig. 1). This would require that the initial ${ }^{14} \mathrm{~N} /{ }^{15} \mathrm{~N}$ at stellar birth was roughly 50, while the lowest values observed astronomically are around 100 (in the Large Magellanic Cloud) [117]. The astronomical data concerning the time evolution of ${ }^{14} \mathrm{~N} /{ }^{15} \mathrm{~N}$ in the Galaxy (summarized in [118]) neither support nor rule out such low ${ }^{14} \mathrm{~N} /{ }^{15} \mathrm{~N}$ in stars that produced the $\mathrm{SiC}$ grains. The Galactic disc today shows generally higher ${ }^{14} \mathrm{~N} /{ }^{15} \mathrm{~N}$ than solar, with a wide scatter of 200-600, and the Galactic center has a lower limit of 600 [118]. These facts suggest that ${ }^{14} \mathrm{~N} /{ }^{15} \mathrm{~N}$ increases with astration, whereas the gradient of ${ }^{14} \mathrm{~N} /{ }^{15} \mathrm{~N}$ with distance from the Galactic center within the disc suggests the opposite trend. We note that the recent factor-of-two reduction in the ${ }^{14} \mathrm{~N}(p, \gamma){ }^{15} \mathrm{O}$ rate based on measurements at LUNA [119] and TUNL [120] [121] further increases the amount of ${ }^{14} \mathrm{~N}$ in the dredged-up material and exacerbates the ${ }^{14} \mathrm{~N} /{ }^{15} \mathrm{~N}$ problem.

All of these considerations bear on the case without CBP. The addition of CBP during the AGB phase (and earlier, during the RGB phase) further increases ${ }^{14} \mathrm{~N} /{ }^{15} \mathrm{~N}$ so that almost none of the observed grains are accessible with CBP if the initial ${ }^{14} \mathrm{~N} /{ }^{15} \mathrm{~N}=\left({ }^{14} \mathrm{~N} /{ }^{15} \mathrm{~N}\right)_{\odot}$. This was discussed by Huss et al. [122] who suggested that the ${ }^{18} \mathrm{O}(p, \alpha){ }^{15} \mathrm{~N}$ cross section might be grossly wrong. 
This does not appear to be likely. The most direct solution to this severe problem would be that ${ }^{14} \mathrm{~N} /{ }^{15} \mathrm{~N}$ in the ISM, at the time the stars parent of the circumstellar grains formed, was at least a factor of 5 - 10 lower than in the sun, so that the assumed initial isotopic composition of $\mathrm{N}$ in all models is incorrect. More direct observations of ${ }^{14} \mathrm{~N} /{ }^{15} \mathrm{~N}$ are clearly required in the proper metallicity domain. In general, the assumption of solar abundances for the precursor stellar sources cannot be valid.

\section{Intermediate Mass Stars (IMS)}

In AGB stars the formal H-burning shell (where maximum energy generation from hydrogen burning occurs) is always a radiative zone. However, for IMS models, convection from the envelope extends into the top of the broad layer where physical conditions are suitable for proton captures. The temperature of the $\mathrm{H}$ shell is very high in these cases (above $10^{8} \mathrm{~K}$ ) and the bottom of the convective envelope can reach temperatures of several $10^{7} \mathrm{~K}$. Here burning occurs in fully convective conditions, so that the consumed H-fuel is continuously replenished and the efficiency of nucleosynthesis becomes quite high. It occurs primarily through the $\mathrm{CNO}$ cycle, but also through reactions involved in the Ne-Na and $\mathrm{Mg}-\mathrm{Al}$ cycles, so that these layers are a suitable site for production of several nuclei, from ${ }^{7} \mathrm{Li},{ }^{13} \mathrm{C},{ }^{14} \mathrm{~N}$, up to ${ }^{23} \mathrm{Na}$. Further, the isotopic mix of $\mathrm{Al}$ and $\mathrm{Mg}$ can be heavily affected, and ${ }^{26} \mathrm{Al} /{ }^{27} \mathrm{Al}$ ratios close to unity may result [123]. This is contrary to what occurs in LMS experiencing CBP where there is no fuel replenishment or extra $\mathrm{H}$ burning.

This process is called Hot Bottom Burning (HBB), following a prediction by Renzini and Voli [124] made well before any stellar model could confirm their hypothesis. The exact mass at which HBB is found in evolutionary codes is dependent on the modeling. There is a consensus that stars of $Z=Z_{\odot}$ experience HBB above $\sim(5-5.5) M_{\odot}$, and that this mass limit decreases with metallicity, so that for population II stars, the phenomenon may be found at masses as low as 3 to $4 M_{\odot}$. Concerning the nucleosynthesis processes of relevance here for short-lived nuclei, the results by Karakas and Lattanzio [123] and Karakas [125] confirm the expectation that HBB produces ${ }^{26} \mathrm{Al}$ at higher efficiency than CBP. Due to the high ${ }^{12} \mathrm{C}$ consumption in the $\mathrm{CNO}$ processing, however, HBB inhibits the formation of a $\mathrm{C}$ star (except in very special cases), so that these high ${ }^{26} \mathrm{Al} /{ }^{27} \mathrm{Al}$ ratios must be associated only with O-rich compositions. A circumstellar grain of $\mathrm{MgAl}_{2} \mathrm{O}_{4}$ with shifted $\mathrm{Mg}$ isotope composition that is consistent with HBB has been found [126]. 


\section{Estimates of the ISM inventory of short-lived nuclei}

In the simplest possible model of galactic nucleosynthesis for a system evolving for a time duration $T$, the inventory of radioactive nuclide $R$ relative to a stable nuclide $I$ uniformly produced in the same process is:

$$
\left[N^{R}(T) / N^{I}(T)\right]_{U P} \simeq \frac{P^{R} p(T) \bar{\tau}_{R}}{P^{I}<p>T}
$$

Here UP refers to uniform production (in the galaxy), $P^{I}\langle p\rangle$ is the average stellar production rate of $I$ over time $T$ and $P^{R} p(T)$ is the production rate of $R$ near the time when production ceased [127]. If we assume that $p(T)$ is constant, then we may calculate relative abundances in such an ISM just using the values of $\bar{\tau}_{R}$, the duration $\left(T \sim 10^{10} \mathrm{yr}\right)$ and estimates of $P^{R} / P^{I}$. All UP calculations were done using the explicit equation:

$$
N^{R}(T)=P^{R} \bar{\tau}_{R}\left(1-e^{-T / \bar{\tau}_{R}}\right)
$$

This should serve as a guide. If the nuclei selected (for each pair $R, I$ ) are typically produced at the same site in a source, we may then obtain rather well-defined estimates of the resulting abundance ratios depending on the assumed nuclear physics. If the ISM is isolated from further production of nuclei at some time prior to onset of formation of the solar nebula, then the time interval between injection and the formation $\left(\Delta_{1}\right)$ results in further decay by the factor $e^{-\Delta_{1} / \bar{\tau}_{R}}$.

This approach gives some insights; however, it is a bit deceiving as the events are discrete ones, not continuous. Let us consider the events of type "a", producing $R_{a}$ and $I_{a}$ nuclei, with a fixed recurrence interval of $\delta_{a}$, and where the yields of nuclei in a single stellar event are $\tilde{p}^{R_{a}}$ and $\tilde{p}^{I_{a}}$. The appropriate scaling factor is the ratio of the galactic time scale to the recurrence time, $T / \delta_{a}$, which is the number of events (typically $10^{2}-10^{3}$ ).

Then we obtain:

$$
N^{R_{a}} / N^{I_{a}}=\tilde{p}^{R_{a}} / \tilde{p}^{I_{a}}\left(\frac{\theta \delta_{a}}{T}+\frac{\bar{\tau}_{R}}{T}\right)
$$

for $\delta_{a} / \bar{\tau}_{R}<<1$, and where $\theta=0$ or 1 depending on whether the sampling time is before or just after the last contributing event. In this case the first term is negligible whether $\theta=0$ or 1 . 
For many nuclei with $\bar{\tau}_{R} \leq 10^{7} \mathrm{yr}, \delta_{a} / \bar{\tau}_{R} \gtrsim 3$, and hence we obtain the following expression:

$$
N^{R_{a}} / N^{I_{a}}=\tilde{p}^{R_{a}} / \tilde{p}^{I_{a}} \frac{\delta_{a}}{T}\left(\theta+e^{-\delta_{a} / \bar{\tau}_{R}}\right)
$$

If $\theta=1$, then the second term with the exponential becomes negligible. It is thus the granularity of the production that governs the inventory of the shorter-lived nuclei [128]. For many of these short-lived nuclei, the inventory is controlled by the last event contributing to the ISM. In this case, if the ISM is sampled at a time $\Delta_{1}$ after the last event:

$$
N^{R_{a}} / N^{I_{a}} \sim \tilde{p}^{R_{a}} / \tilde{p}^{I_{a}} \frac{\delta_{a}}{T} e^{-\Delta_{1} / \bar{\tau}_{R}}
$$

Thus, these nuclei must in some way be considered as resulting from late injection.

For the abundances in the ISM using the UP model and eqn. 2 we used estimates of the production rates of both the radioactive $(R)$ and stable $(I)$ nuclei. All pertinent ratios for UP are given in Table 1 for the cases $\Delta_{1}=0$, $5 \mathrm{Myr}, 10 \mathrm{Myr}$, and $70 \mathrm{Myr}$, where $\Delta_{1}$ is the time after the termination of uniform production and $e^{-\Delta_{1} / \bar{\tau}}$ corrects for the subsequent decay of isotope $R$ without any additional injection into the ISM. For the actinides we counted progenitors following [129] and [130], and obtained $\left({ }^{244} \mathrm{Pu} /{ }^{232} \mathrm{Th}\right){ }_{I S M}^{U P}=6 \times$ $10^{-3}$ and ${ }^{247} \mathrm{Cm} /{ }^{232} \mathrm{Th}=1.1 \times 10^{-3}$ (see [131] and section 11 in this paper). For $\mathrm{Ca}, \mathrm{Pd}$, I and Cs we adopted the choices discussed in [84]. If we consider ${ }^{26} \mathrm{Al}$, then $\left({ }^{26} \mathrm{Al} /{ }^{27} \mathrm{Al}\right)_{I S M}^{U P} \sim p^{26} \mathrm{Al} / p^{27} \mathrm{Al} \times 10^{-4}$. As ${ }^{26} \mathrm{Al}$ is produced at a very low level $\left(p^{26 \mathrm{Al}} / p^{27} \mathrm{Al} \sim 10^{-3}-10^{-2}\right)$ in SNe II and other sources, the ISM value will be extremely low. The inventory of ${ }^{26} \mathrm{Al}$ in the Galaxy today $\left({ }^{26} \mathrm{Al} \sim\right.$ $2-3 M_{\odot}$ ) is obtained from $\gamma$-ray measurements (see a review in [132]). This gives an observed ratio of $\left({ }^{26} \mathrm{Al} /{ }^{27} \mathrm{Al}\right)_{\mathrm{ISM}} \approx 6 \times 10^{-6}$; we can notice that the continuous production computed using present SN models cannot match this, but is low by at least an order of magnitude. For the cases of ${ }^{60} \mathrm{Fe}$ and ${ }^{53} \mathrm{Mn}$, we may use the calculations from SNe II. Yields for these two nuclei have been calculated by both Woosley \& Weaver [133] and Rauscher et al. [134], and are in rather good agreement, with about the same values being obtained by these workers for both $15 M_{\odot}$ and $25 M_{\odot}$ stars. For ${ }^{135} \mathrm{Cs},{ }^{129} \mathrm{I}$, and ${ }^{107} \mathrm{Pd}$, the $p^{R} / p^{I}$ ratios are close to unity [84] [131]. For ${ }^{41} \mathrm{Ca}$ we used a nominal value of $p^{41} \mathrm{Ca} / p^{40} \mathrm{Ca} \sim 10^{-3}$. For ${ }^{36} \mathrm{Cl}$ we used $p^{36} \mathrm{Cl} / p^{35} \mathrm{Cl} \approx 10^{-2}$ from [134]. Note that each nuclide in an isotopic pair may represent different astrophysical sites and processes. In many cases stellar sources are not well established.

We list nominal values for the observed abundances in the ESS in the fifth column of Table 1. These are essentially the same as given in [84]. Comparison 
of these determinations with the calculated UP values may be used as a guide as to which nuclei might be available in the general ISM and which require special sources. We will discuss critical aspects of the measured values in meteorites in a later section.

If we now compare the values for UP with the ESS measurements, it is evident that, between the actinides and ${ }^{53} \mathrm{Mn}$, the estimated ISM inventory is approximately sufficient to provide the observed abundances for many short-lived nuclei. For ${ }^{26} \mathrm{Al},{ }^{41} \mathrm{Ca}$, and ${ }^{10} \mathrm{Be}$, the model of uniform galactic nucleosynthesis is insufficient, particularly if the time scale prior to solar system formation is $\sim 10^{6}$ years. It follows that, if $\left({ }^{26} \mathrm{Al} /{ }^{27} \mathrm{Al}\right)_{E S S}=5 \times 10^{-5}$, then the material of the solar system did not form from the average ISM, but must have sampled a hot spot.

As ${ }^{10} \mathrm{Be}$ can not be produced by stellar nucleosynthesis, it must come from some irradiation process. It is therefore these three nuclei that require some very late addition.

Evidence for ${ }^{129} \mathrm{I}$ is found in a wide variety of ESS materials and even in terrestrial gas samples. There is a range of ${ }^{129} \mathrm{I} /{ }^{127} \mathrm{I}$ found in meteorites (cf. review by Ott [135] and report by Whitby et al. [136]). We immediately see that ${ }^{129} \mathrm{I}_{U P}$ is grossly overproduced (by a factor of 23 to 50 ) as compared to the observed ${ }^{129} \mathrm{I} /{ }^{127} \mathrm{I}$ ratios. ${ }^{129} \mathrm{I}$ is a pure $r$-process product [137] [138] and cannot be produced in the $s$ process [139] [97] [131]. This has long been recognized and is the basis of the argument that the last " $r$ process" source enriching the local ISM was $\Delta_{1}\left({ }^{129} \mathrm{I}\right) \sim(0.7$ to 1$) \times 10^{8}$ yr before the solar system formed (see Table 1). The more recent discovery of ${ }^{182} \mathrm{Hf}$ and its abundance in the ESS (cf. [34] [35] [36] [37]) and important new results by Yin et al. [140] and Kleine et al. [141] led to a further discrepancy with ${ }^{129} \mathrm{I}$. The ${ }^{182} \mathrm{Hf}$ nuclide cannot be significantly produced in AGB stars and must be completely dominated by an $r$-process source [97] [131]. There is no time interval $\Delta_{1}$ that will allow the two $r$-process nuclei, ${ }^{182} \mathrm{Hf}$ and ${ }^{129} \mathrm{I}$, to be in agreement. This then required that the $r$-process was not a single process producing both ${ }^{129} \mathrm{I}$ and ${ }^{182} \mathrm{Hf}$ but now had to be considered as two or more processes, one of which produces low mass $r$-nuclei (at or below Ba) while the other source produces heavy $r$-nuclei (Ba and above) as proposed by Wasserburg, Busso \& Gallino [131]. It then developed that the common and long-time practice of attributing the $r$-process site to any standard SNe II has major flaws. Observations on low metallicity halo stars show clearly that heavy $r$-process nuclei are not associated with any production of all the nuclei between oxygen and germanium. This includes the "iron" peak, which was usually assumed to be co-produced with the $r$-nuclei. This result was implied by the early observations by McWilliam et al. [142] and [143]. Much more extensive work on low-metallicity halo stars (cf. [144] [145] [146]) showed that Fe-group and other nuclei with $A<130$ were fully decoupled from heavy $r$-process nucleosynthesis (including $\mathrm{U}$ and $\mathrm{Th}$ ) [147] 
[148]. This then requires that those SNe II that may be the site of a heavy $r$ process must have masses $8 M_{\odot} \leq M \leq 11 M_{\odot}$ (with small envelopes). Another possibility is that the heavy $r$-nuclei might be the result of $A I C$ - accretioninduced core collapse - (to make a neutron star with concurrent production of large neutrino fluxes), or even possibly be related to explosive nucleosynthesis on white dwarfs by some other mechanism (see [148] and references therein). The source of some of the "light" r-process nuclei is also a matter of current study [149]. Basic issues and aspects of the "r-process" are discussed by [150][151][152][153]. For the contributions of Type Ia SNe, see [154]. The $r$-processes, and their sites and sources, are thus still not fully understood or identified (see review by Qian [155]). We further wish to emphasize the point that the ratio $\mathrm{Th} / \mathrm{Eu}$ is not constant in the different $r$-processes, but there is a distinct change in yields between $\mathrm{Eu}$ and Th. This then invalidates the often-used $\mathrm{Th} / \mathrm{Eu}$ chronometer, as pointed out by Qian [156]. Those inferences about the yield pattern involving very heavy $r$-nuclei and those of intermediate mass are confirmed in this work (see Section 13).

Although ${ }^{107} \mathrm{Pd}$ is found to be abundant in the UP model for $\Delta_{1}=5 \times 10^{6} \mathrm{yr}$, it is grossly underabundant if the time interval $\Delta_{1}^{107} \mathrm{Pd} \sim \Delta_{1}^{129} \mathrm{I} \sim 7 \times 10^{7}$ yr as inferred from ${ }^{129} \mathrm{I}$. If ${ }^{107} \mathrm{Pd}$ is from the $r$-process, it is most plausibly produced with the lower mass $r$-process nuclei along with ${ }^{129} \mathrm{I}$, possibly also with the Fe group. ${ }^{107} \mathrm{Pd}$ should then be absent as a residue of long-term galactic nucleosynthesis. It was a version of this conflict that led to the joint effort of the Torino and Caltech groups to seek a solution considering a late AGB injection, since ${ }^{107} \mathrm{Pd}$ is readily produced in these stars. This led to connections and predictions for the much shorter-lived nuclei produced by AGB stars. For these AGB contributions there will also be ${ }^{60} \mathrm{Fe}$ and other nuclides produced that will add on to the possible ISM inventory. From the above arguments it follows that radioactive iron group nuclei (e.g. ${ }^{53} \mathrm{Mn}$ and ${ }^{60} \mathrm{Fe}$ ) are not to be associated with the heavy $r$-process nuclei. If they are associated with the light $r$-nuclei then they should also be extinct if $\Delta_{1} \sim 7 \times 10^{7} \mathrm{yr}$. This leaves a problem with ${ }^{53} \mathrm{Mn}$ as it cannot be produced in AGB stars (see Section 9).

The lifetime of ${ }^{244} \mathrm{Pu}$ is so long that small changes in the history $\left(\Delta_{1}\right)$ play no role if $\Delta_{1}<<10^{8} \mathrm{yr}$. We first note that using the standard estimated yield for the actinides, the calculated value of $\left({ }^{244} \mathrm{Pu} /{ }^{232} \mathrm{Th}\right)_{I S M}^{U P}$ is within a factor of two greater than that found in the ESS. This is fully consistent with the transactinides being part of the general ISM inventory from which the solar system formed. For ${ }^{247} \mathrm{Cm}$ there is only an upper bound on its abundance. The experimental approach used is to find variations in ${ }^{235} \mathrm{U} /{ }^{238} \mathrm{U}$ caused by variations in the $\mathrm{Cm} / \mathrm{U}$ ratios in different phases in meteorites and different meteorite samples. The strict interpretation of the results depends on estimates of the chemical fractionation of $\mathrm{Cm}$ from $\mathrm{U}$. There is, of course, some basic uncertainty in the estimated nucleosynthetic yield of these nuclei. The study by Chen \& Wasserburg [157] [158] gave an upper limit of $\left({ }^{247} \mathrm{Cm} /{ }^{235} \mathrm{U}\right)_{E S S}<2 \times 10^{-3}$. A 
new report by Stirling et al. using more precise techniques on bulk meteorites but with very limited $\mathrm{U} / \mathrm{Nd}$ elemental fractionation for chondrites, gives a bound of $1.0 \times 10^{-4}[159]$. The most direct interpretation of the results is that ${ }^{247} \mathrm{Cm}$ is in low or very low abundance compared to the UP model. As mentioned, the measured abundance of ${ }^{244} \mathrm{Pu}$ is low by a factor of two compared to the UP value. This would then appear to require a significant time interval between the termination of heavy $r$-nucleosynthesis and formation of the solar nebula if the counting of precursors is a reasonably reliable estimate of the relative yields for actinides. We will return to this matter in the discussion.

For the actinides ${ }^{238} \mathrm{U},{ }^{235} \mathrm{U},{ }^{232} \mathrm{Th}$, and ${ }^{244} \mathrm{Pu}$, the observed abundances are certainly within a factor of two of those anticipated from the UP model (cf. [131]). Further, ${ }^{146} \mathrm{Sm}$ is in accord with the observations, although its stellar origin still remains somewhat uncertain. As both ${ }^{146} \mathrm{Sm}$ and ${ }^{144} \mathrm{Sm}$ are $p$ process nuclei, the estimated production ratio should not be in gross error. The $p$ nuclide ${ }^{92} \mathrm{Nb}(\bar{\tau} \sim 52 \mathrm{Myr})$ was discovered in the ESS by Harper [29], with ${ }^{92} \mathrm{Nb} /{ }^{93} \mathrm{Nb} \sim 0.7 \times 10^{-5}$. We do not have any basis for inferring precise yields for this nuclide, as estimates of ${ }^{92} \mathrm{Nb} /{ }^{93} \mathrm{Nb}$ are widely diverse, and further the index isotope, ${ }^{93} \mathrm{Nb}$, is almost pure $s$-process.

The ${ }^{182} \mathrm{Hf}$ abundance has been revised [140] [141] to the value $\left({ }^{182} \mathrm{Hf} /{ }^{180} \mathrm{Hf}\right)_{E S S}=$ $1.0 \times 10^{-4}$. These workers have established the abundance of ${ }^{182} \mathrm{Hf}$ in the ESS and the initial reference ratio for bulk solar $\mathrm{W}$ isotopes. The UP value is a factor of 4 greater than the ESS value and also appears to require some time interval for decay $\left(\Delta_{1} \sim 18 \mathrm{Myr}\right)$. In general, all of the heavy $r$ nuclei appear roughly consistent with long term galactic nucleosynthesis with no requirement of significant very late addition from a special source. The general inferences given above are in accord with those in [131]. Some significant time interval $\Delta_{1}$ appears to be required in order to obtain a plausible match to the observations of the shorter-lived heavy $r$-nuclei. This is an important matter as it generates further conflicts (see Section 13).

\section{$7 \quad$ Injection from a single stellar source}

We now consider a model of injection of freshly synthesized nuclear material from a single stellar source into the protosolar molecular cloud. It is assumed that the pre-existing material and the injected material are reasonably wellmixed. Then the equations governing the mixture for short-lived nuclei (taken originally to be absent) are as follows.

Let $N_{S C}^{R}$ be the number of short-lived radioactive nuclei $R$ in the solar cloud (SC); $N_{S C}^{I}$ be the number of stable $I$ nuclei (of the same element as $R$ ) in the protosolar cloud. Note that in some cases we use another radioactive nuclide as 
an index isotope for the ratio (e.g. ${ }^{235} \mathrm{U} /{ }^{238} \mathrm{U}$ ), and this changes the formalism because decay must be considered. $N_{E N V}^{R}, N_{E N V}^{I}$ are the numbers of nuclei $(R$ and $I)$ in matter in the stellar envelope that are injected into the solar cloud. Then $N_{S C}^{I}$ is the sum of the previous ISM inventory $\left(N_{0}^{I}\right)$ and of the late stellar addition $\left(N_{E N V}^{I}\right)$, while for short-lived nuclei $N_{S C}^{R}$ contains only the stellar contribution $\left(N_{E N V}^{R}\right)$. One has:

$$
\begin{aligned}
\frac{N_{S C}^{R}}{N_{S C}^{I}} \equiv \alpha_{0}^{R, I} & =\frac{N_{E N V}^{R}}{N_{0}^{I}+N_{E N V}^{I}} \\
& =\frac{\left(N^{R} / N^{I}\right)_{E N V} N_{E N V}^{I}}{N_{0}^{I}+N_{E N V}^{I}} \\
& =\frac{\left(N^{R} / N^{I}\right)_{E N V} q_{E N V}^{I} M_{E N V}}{q_{0}^{I} M_{0}+q_{E N V}^{I} M_{E N V}}
\end{aligned}
$$

We define $q_{E N V}^{I}$ and $q_{0}^{I}$ to be the number of stable $I$ nuclei per gm of matter in the envelope and in the polluted ISM cloud. Note that the $q$ values depend on metallicity. For the case where the ejected stellar envelope is not well-mixed, the problem is more complex: in that case the terms $\left(N^{R} / N^{I}\right)_{E N V} q_{E N V}^{I}$ must be summed over all the contributing subunits of the parent star. This can be especially important for a SNII. Thus,

$$
\frac{\left(N^{R} / N^{I}\right)_{E N V}}{\alpha_{0}^{R, I}} \frac{q_{E N V}^{I}}{q_{0}^{I}} \approx \frac{M_{0}}{M_{E N V}} \equiv 1 / f_{0}
$$

This is the condition obtained at the time of injection and instant mixing with the local ISM. It relates the ratio of the radioactive nuclide $R$ to the stable nuclide $I$ in the envelope to that in the unpolluted cloud. The term $M_{0} / M_{E N V}$ is then the ratio of the mass of the cloud to the mass of injected stellar envelope. Note that the term $f_{0}$ is the dilution factor and will be used later. This follows the treatment in [97] with some modification as they used the ratios in the He shell, not in the envelope.

For a self-consistent solution for a variety of isotopic pairs $(R, I)$, it follows that the right hand side of equation (7) should be the same for all $\left(N^{R} / N^{I}\right)$. This is a rather strict constraint and connects the relative abundances in the hypothesized stellar envelope to the initial values in the polluted protosolar cloud. Note that all the terms on the left hand side are only dependent on the model of stellar nucleosynthesis. A model which must assume a separate stellar source for each radioactive isotope " $R$ " is certainly not attractive. Such proposals are commonly found in the literature. The approach used here will be to search for a self-consistent solution for several radioactive nuclei. However, it will become evident that a single source can not provide the observed results 
and that a blend is required.

For a model to be reasonable, the dilution factor $f_{0}$ must be the same for all species and the times $\Delta_{1}^{R}$ for each radioactive nucleus " $R$ " should be selfconsistent (no negative values), and physically compatible with the values of

$\alpha_{o b s}^{R, I}$. These rules apply to any model of injection by a single source with subsequent mixing. The degree of homogenization need not be complete, and disagreements between the model and observed abundances by factors of say $\sim 2$ may be acceptable considering the uncertainties. Factors as large as 10 or more are not compatible with this approach nor with the observations.

\section{Meteoritic observations on short-lived nuclei}

Samples of meteorites are the principal source for determining the presence and the abundances of short-lived nuclei in the early solar system. Meteorites represent objects formed early in solar system history when low mass objects (protoplanets) accumulated from condensed material with some volatile elements. They may be aggregates of material processed chemically and thermally within the solar system and mixed together with a small amount of residual presolar grains that survived the processing. Material aggregated from a heated portion of the solar nebula would thus contain such a mixture. A small planet aggregated from original presolar grains and which was then heated and partly melted would also produce highly heterogeneous materials in different zones depending on the wide range of degrees of chemical processing and preservation of the original material. Even the most "primitive" meteorites show evidence of extensive chemical and thermal processing. They represent "averages" of bulk solar material with only a small amount of preserved presolar grains (cf. [160]). The new results on presolar silicate grains [76] indicate that the net abundance of presolar grains is much higher in unmetamorphosed meteorites. As yet no macroscopic object has been found that is simply a mechanical aggregate of unprocessed presolar material.

The small objects in chondrites, which were melted and crystallized at some early time, would have locally chemically fractionated phases that crystallized together. These would show correlated isotopic enrichments of the daughter isotope due to the radioactive decay of the parent in different parts of the same small object. It is this chemical fractionation between mother and daughter element, occurring while some short-lived nuclei are still abundant, that gives direct evidence of both the presence and abundances of these nuclei at that time and place. The time and place is, in general, not known. The bulk chemical composition and morphology of some objects (CAIs, chondrules) defines the type of chemical-physical processing. It does not define a time or place. The most common assumption is that these objects formed in the solar neb- 
ula, necessarily at very high dust-to-gas ratios [161] [162]. They may, in fact, be produced from a protoplanet. Attributing objects to direct formation in the solar nebula may not clarify the issues at hand. We note that the accretion disks found around stars in the early stages of formation are, in general, cool. The only zones that show evidence of being "hot" are those very close to the star. In no cases are temperatures of $10^{3} \mathrm{~K}$ observed in any part of the disk. As a result, it is not evident that a hot solar nebula can be assumed for formation of the high temperature materials observed in chondrules (including, and in particular, CAIs). However, there are more developed views of the thermal structure of the solar nebula (see [163]). The X-wind scenario of Shu, Shang, \& Lee [164] would certainly provide a hot processing zone. It was proposed by Wood [165] that shock heating could play a major role in both CAI and chondrule formation. The significance of shock wave heating and the dynamics of formation of chondrules in the solar nebula has become a focus of serious study [166][167][168][169]. It is possible that the nanodiamonds (which have bulk solar ${ }^{13} \mathrm{C} /{ }^{12} \mathrm{C}$ but have been considered as presolar grains by many workers) may be products of shock in the solar nebula. NanoSIMS analysis may aid in clarifying this issue if evidence of large ${ }^{12} \mathrm{C} /{ }^{13} \mathrm{C}$ variations can be found in the somewhat reduced sampling volumes available by this technique. The protoplanets heated by decay of ${ }^{26} \mathrm{Al}$ and ${ }^{60} \mathrm{Fe}$ would certainly provide a high temperature planetary regime. The formation of CAIs and chondrules remains a fundamental problem of meteoritics. Assuming them to be products of nebular processes may in some cases lead us astray. The "primitive" objects (e.g. chondrites) were parts of asteroids which are small planetary bodies. They will, to varying degrees, show some effects of planetary metamorphism. If they were from very small bodies or near the surface of small planetary bodies, they would have undergone less thermal and chemical processing.

Alternatively, the meteorite may represent extensive melting and chemical segregation of protoplanets (cores, mantles, crust). They would be essentially homogenized isotopically and only show the effects of any remaining shortlived nuclei by isotopic enrichments of the daughter isotope after there is large chemical fractionation on a planetary scale. The material in these protoplanets will also undergo long term heating after the planet formed. The two time scales (to form "primitive" objects and to form planetary differentiates, etc.) may not in general be identifiable or resolvable. The major protoplanetary differentiation events are plausibly presumed to occur later, after aggregation of the dust.

The relative abundance of a radioactive nuclide $R$ relative to a stable isotope $I$ as observed in some meteoritic material is defined as $\alpha_{o b s}^{R, I}$. Different objects may show different values of $\alpha_{o b s}^{R, I}$. As time passes in going from some assumed initial state of the protosolar nebula (with the original values $\alpha_{0}^{R, I} e^{-\Delta_{1} / \bar{\tau}_{R}}$ ) to the state when the particular meteoritic material formed, the relationship 
between these two values is given by

$$
\alpha_{o b s}^{R, I}=\alpha_{0}^{R, I} e^{-\Delta_{2} / \bar{\tau}_{R}-\Delta_{1} / \bar{\tau}_{R}}
$$

where $\Delta_{2}$ is the time interval from the initial state $\left(\Delta_{2}=0\right)$ to the time of formation of the differentiated object. There are no direct measurements of $\Delta_{1}$ or $\Delta_{2}$.

It is possible to measure the age $(T)$ of formation (isotopic equilibration) of an object relative to the present time. More ancient objects should better record short-lived nuclei. Thus there should be a correlation of age with the abundance $\alpha_{o b s}^{R, I}$ of a radioactive nuclide $R$. Even in the cases where there appears to be a correlation, this does not fix the time $T_{0}$ when $\Delta_{2}=0$ (i.e., the initial state of the solar nebula). Note that for a long-lived nuclide $\left(\bar{\tau} \gtrsim 10^{9}\right.$ yr), an accuracy of $\sim 10^{6}$ years out of 4.56 Gyr requires that the lifetime be known to better than $\sim 0.1 \%$. There are no absolute lifetimes known to that level of precision. The best that can be done is to use self-consistent ages by a particular long-lived parent-daughter system. The most useful of these is the ${ }^{207} \mathrm{~Pb}-{ }^{206} \mathrm{~Pb}$ method based on an assumed fixed ${ }^{235} \mathrm{U} /{ }^{238} \mathrm{U}$ ratio and assuming closed system behavior. This method uses rather precisely measured $\mathrm{Pb}$ isotopic ratios, not elemental abundances. However, it is not possible to demonstrate closed system behavior from the ${ }^{207} \mathrm{~Pb}-{ }^{235} \mathrm{U}$ and ${ }^{206} \mathrm{~Pb}-{ }^{238} \mathrm{U}$ systems at the level of precision required, even ignoring uncertainties in decay constants, analytical data, and model assumptions. It is, for the most part, the presence of the short-lived species that indicates that the object formed at a value of $T$ that is close to the initial state. However, major efforts at determining a chronology based on the ${ }^{207} \mathrm{~Pb}^{206} \mathrm{~Pb}$ system have led to important advances and appear to provide valuable information [158] [170] [171] [172]. In seeking to obtain precise ages of formation, the problems of properly recognizing later metamorphism, element redistribution, and the accretion of matter that had previously been metamorphosed, still remain and must be addressed (cf. [173]). The complexities of obtaining precise ${ }^{207} \mathrm{~Pb}^{206} \mathrm{~Pb}$ meteorite ages is extensively discussed by Tera and Carlson [174] and Tera, Carlson, and Boctor $[175]$.

For the short-lived nuclei, we are therefore left with a set of values, $\alpha_{o b s}^{R, I}\left(\Delta_{i}^{R}\right)$ $(i=1,2)$, corresponding to different values of $\Delta_{1}$ and $\Delta_{2}$ which are not known. If, from independent arguments, we can assume that $\left(\Delta_{1}^{R^{*}}+\Delta_{2}^{R^{*}}\right)<<\bar{\tau}_{R^{*}}$ for some nuclide $R^{*}$, then the value of $\alpha_{o b s}^{R^{*}, I} \approx \alpha_{0}^{R^{*}, I}$. Then the relationship between the dilution factor and the abundances in the stellar envelope for $R^{*}$ and $I$ for a given stellar model is known from equation 7 . This then fixes the dilution factor for all the other nuclei $R$ for that stellar model. Given a proposed stellar model for the contaminating source, $\alpha_{0}^{R^{*}, I}$ is determined by the dilution factor. All shorter-lived species should be explained by the model with the same dilution factor for all species with positive $\Delta_{i}(R)$. 


\section{What do SNe II Produce?}

\subsection{A SNe II source for ${ }^{26} \mathrm{Al},{ }^{41} \mathrm{Ca},{ }^{53} \mathrm{Mn}$, and ${ }^{60} \mathrm{Fe}$}

The issues to be discussed here are the questions of which short-lived nuclei in the ESS might have been supplied by a 'local' triggering supernova and whether there are diagnostic characteristics that would identify SN contributions. We will take the diverse, and generally unmixed ejected material from a type II SN source as representing the bulk composition of the dispersed ejecta after the explosion. We denote this composition with subscript "ENV" in analogy to our notation for lower mass stars. We will not discuss the $r$-process contributions because of the need for diverse $r$-sources and also because of the questions relating to SNe II as sources of heavy $r$-nuclei as discussed earlier. Our focus will be on the lighter nuclides produced by SNe II following available models. There are direct observational data showing that SNe II produce the Fe group nuclei (including, and in particular, ${ }^{56} \mathrm{Ni}$ ). In an earlier report [176], following the work of Timmes et al. [177], we further investigated the problem of whether ${ }^{26} \mathrm{Al},{ }^{41} \mathrm{Ca},{ }^{60} \mathrm{Fe}$ and ${ }^{53} \mathrm{Mn}$ could be adequately provided by a SN II event, using the models of Woosley \& Weaver [133] as a basis. As recognized by [176] and [177], the average number ratio of $\left({ }^{26} \mathrm{Al} /{ }^{60} \mathrm{Fe}\right)_{E N V}$ in the SN ejecta of these models is about 8.6, from a wide range of stellar models, the only clear exception being for $M \simeq 13 M_{\odot}$. It was proposed that the $\left({ }^{26} \mathrm{Al} /{ }^{60} \mathrm{Fe}\right)$ ratio be used as a test of the SN II model. It was found that to match the $\left({ }^{26} \mathrm{Al} /{ }^{27} \mathrm{Al}\right)_{\Delta_{1}}$ ratio of $\sim 5 \times 10^{-5}$, and the $\left({ }^{41} \mathrm{Ca} /{ }^{40} \mathrm{Ca}\right)_{\Delta_{1}}$ ratio of $\sim 1.5 \times 10^{-8}$, a mixing ratio of $\sim 3 \times 10^{-4}$ was required and that this source yielded $\left({ }^{60} \mathrm{Fe} /{ }^{56} \mathrm{Fe}\right)_{\Delta_{1}} \sim 10^{-6}$. In addition, the inferred relative abundance of ${ }^{53} \mathrm{Mn}$ implies that $\left({ }^{53} \mathrm{Mn} /{ }^{55} \mathrm{Mn}\right)_{\Delta_{1}}$ is about $10^{-3}$. This is far above the observed value shown in Table 1. A SN II model would also require that several percent of all ${ }^{16} \mathrm{O}$ in the protosolar cloud, as well as large contributions of other major isotopes, was from a single SN II event.

The yields of nuclear species produced in models of SNe II have been extensively studied by many workers (cf. [133] [178] [134] [179]) and define a rather self-consistent set of results for major elements. In particular, a new generation of SN II nucleosynthesis models was computed by Rauscher et al. [134] for a wide range of masses. An up-to-date set of reaction rates (both experimental and theoretical) was used, in conjunction with upgrades in the evolutionary code. These calculations did not include a parametric $r$-process model but did treat neutron capture reactions during the normally-occurring intermediate and advanced evolutionary stages. Explosive nucleosynthesis from a parameterized final collapse and bounce process was also included. As a result, several neutron capture nuclei are produced. In the Rauscher et al. [134] paper, Tables 8 and 9 (published electronically) give the yields of both radioactive and stable 
nuclei. Using these results and the published ejected masses, we have calculated the corresponding $q_{E N V}^{I} / q_{0}^{I}$ and $\left(N^{R} / N^{I}\right)_{E N V}$ values in the ejected bulk envelope, or "wind", of a $15 M_{\odot}$ SN for selected isotopes as shown in Table 2. From these $q_{E N V}^{I}$ values we have calculated the dilution factor $\left(f_{0}\right)$ required to give the $\left({ }^{26} \mathrm{Al} /{ }^{27} \mathrm{Al}\right)_{E S S}$ and the $\left({ }^{41} \mathrm{Ca} /{ }^{40} \mathrm{Ca}\right)_{E S S}$ values. The time scale for ${ }^{41} \mathrm{Ca}$ effectively defines a value for $\Delta_{1}$. The resulting values of $\left(N^{R} / N^{I}\right)_{\Delta_{1}}$ are shown for this couple of $f_{0}$ and $\Delta_{1}$ values. It can be seen that most short-lived nuclei are produced abundantly, with the only exception being ${ }^{107} \mathrm{Pd}$, which is somewhat low. The principal conclusion is that if ${ }^{26} \mathrm{Al}$ and ${ }^{41} \mathrm{Ca}$ are from a SN II source, then the ratio $\left({ }^{60} \mathrm{Fe} /{ }^{56} \mathrm{Fe}\right)_{\Delta_{1}}$ must be very high $\left(\sim 5 \times 10^{-5}\right)$ and the $\left({ }^{53} \mathrm{Mn} /{ }^{55} \mathrm{Mn}\right)_{\Delta_{1}}$ must be extremely high $\left(\sim 3 \times 10^{-3}\right)$, which is implausible. Essentially, the same results are found using the $25 M_{\odot}$ model of Rauscher et al. [134] as shown by [58]. These conclusions are essentially the same as would be obtained with the yields by Woosley \& Weaver [133]; see also [84] and [176].

There is one discrepancy of note that pertains to the ${ }^{26} \mathrm{Al}$ and ${ }^{60} \mathrm{Fe}$ budget. Rauscher et al. [134] give an average ${ }^{26} \mathrm{Al} /{ }^{60} \mathrm{Fe}$ ratio of approximately 1.5. This is lower by a factor of about 5.7 compared to what was previously found by Woosley \& Weaver [133]. The ratio ${ }^{26} \mathrm{Al} /{ }^{60} \mathrm{Fe}$ obtained by Rauscher et al. [134] is now in direct conflict with the limits set by $\gamma$-ray observations [180] [181] [182], which give a steady-state value for the Galaxy in accord with [133]. Recently, new models have been presented by Limongi \& Chieffi [179] in which the ${ }^{26} \mathrm{Al} /{ }^{60} \mathrm{Fe}$ ratios are more similar to those of Woosley \& Weaver [133].

\subsection{The oxygen conundrum}

The addition of major elements (e.g. O, Si, Mg, Fe, Ti, etc.) by an SN II can be seen by considering the fraction $\delta N^{I} / N^{I}$ of the total solar inventory from such a postulated event. This gives $\delta N^{I} / N^{I}=\left(q_{E N V}^{I} / q_{0}^{I}\right) f_{0}$. For major elements like oxygen and iron, $q_{E N V}^{I} / q_{0}^{I} \sim 10^{2}$, so that for $f_{0}=3 \times 10^{-4}, \delta N^{I} / N^{I}=3 \times 10^{-2}$, thus requiring a very substantial fraction of the major elements from a single late source. As indicated in section 2, the overwhelming abundance of presolar grains (both oxide and carbide) are from AGB stars and the hallmarks of SNe II in the grain population are quite uncommon. The only evidence of major isotopic shifts relative to the average "solar" values is in oxygen. The discovery by Clayton et. al. [9] was critical to stimulating work in isotopic studies of meteorites. However, there are no large (few percent level) effects observed in $\mathrm{Mg}, \mathrm{Si}, \mathrm{Ca}, \mathrm{Fe}, \mathrm{Ni}, \mathrm{Sr}, \mathrm{Ba}$, etc., that have been found in related materials. The

${ }^{16} \mathrm{O}$ excess has most frequently been interpreted as the result of injection by a supernova. However, there is no correlation of other isotopic shifts with the ${ }^{16} \mathrm{O}$ enrichment. Maintaining a separate gas reservoir enriched in ${ }^{16} \mathrm{O}$ from a supernova source without any evidence of effects in other nuclei is not readily 
understandable. It follows that the ${ }^{16} \mathrm{O}$ enrichment is not plausibly associated with nucleosynthetic processes; there must exist chemical-physical processes that cause the isotopic shifts. The discovery of ${ }^{16} \mathrm{O}$-enriched oxygen without shifts in ${ }^{17} \mathrm{O} /{ }^{18} \mathrm{O}$ in the laboratory by Thiemens and Heidenreich [183], and in the stratosphere and laboratory by Mauersberger et al. [184] [185] testifies to such processes. These effects, which occur in oxygen-rich environments and which were not explainable by classical isotopic fractionation mechanisms, have now been explained on sound theoretical grounds by Gao, Chen and Marcus [186]. However, the explanation of the oxygen isotopic effects in the high-temperature condensed phases found in meteorites and in bulk meteorite samples remains a serious problem. A complex theoretical model has been proposed by Marcus [187], but this remains to be tested. A classical mechanism of self shielding in the gas phase was early suggested by Navon and Wasserburg [188] as a possible source of the ${ }^{16} \mathrm{O}$ anomaly. These workers showed that the self shielding did not apply to the ozone cases cited above. They also argued that the solar nebula would most plausibly provide a CO-rich environment and that self shielding might provide the isotopic effects observed.

Recognition of the difficulties with a nucleosynthetic source for the ${ }^{16} \mathrm{O}$ anomaly has grown over the years (cf. [189]). As a result, the self shielding mechanism in a CO nebula environment has recently attracted considerable interest by several groups [189] [190] [191] [192] [193]. Because of the importance of self shielding in molecular clouds, this problem is the subject of ongoing study, although there is no clear support for the required oxygen effects [194]. The ${ }^{16} \mathrm{O}$ problem is important and remains unsolved. A key question is: what is the bulk isotopic composition of the sun? It has long been known that the solar isotopic abundance of oxygen is not known precisely enough from astronomical observations. If the bulk solar oxygen is like CAIs, then the remaining planetary material is depleted in ${ }^{16} \mathrm{O}$ relative to the bulk solar system. If it is like average planetary oxygen, then the CAIs require an enrichment in ${ }^{16} \mathrm{O}$. All models must, of necessity, assume an initial oxygen isotopic composition. A recent effort to determine the oxygen isotopic composition of the solar wind by Hashizume and Chaussidon [195] points toward a ${ }^{16} \mathrm{O}$-enriched composition for oxygen implanted by the solar wind on grains of metal from the lunar soil.

In any self shielding or photolysis mechanism, the basic problems are: finding the specific molecular reaction paths necessary to produce the isotopic effects in the gas phase appropriate to the bulk gas composition and molecular speciation; considering the problem of isotopic exchange in the gas phase; finding the means of sequestering fractionated materials; preserving the isotopic effects in condensed phases; and then heating the sequestered condensed material so that the effects are present in the final material - but with back reactions for some phases with "normal" oxygen. This is a complex problem in chemical physics, cosmochemistry and meteoritics. A sequestration by precipitation and removal of icy objects has been suggested [196]. Self shielding 
definitely merits further study, but as yet it is not a "slam dunk" solution to the problem.

\subsection{A SNe II source for only ${ }^{53} \mathrm{Mn}$ and ${ }^{60} \mathrm{Fe}$}

In summary, for a SN II source, if one matches the ESS values of ${ }^{26} \mathrm{Al} /{ }^{27} \mathrm{Al}$ and ${ }^{41} \mathrm{Ca} /{ }^{40} \mathrm{Ca}$, and uses the Rauscher et al. [134] models (see Table 2), one finds the following results:

(i) The mixing ratio of $f_{0}=3 \times 10^{-4}$ corresponds to $3 \times 10^{4} M_{\odot}$ of ISM for $\sim 10 M_{\odot}$ of ejecta. This is roughly compatible with the amount of mass necessary to slow down the SNe II ejecta to local velocities [197]. The time scale $\Delta_{1}$ becomes $\sim 10^{6}$ yr.

(ii) The ${ }^{53} \mathrm{Mn} /{ }^{55} \mathrm{Mn}$ is very far above any of the observed values for the ESS.

(iii) The ${ }^{60} \mathrm{Fe} /{ }^{56} \mathrm{Fe}$ is also far above the existing ESS data.

(iv) The addition of substantial amounts $(\sim 3 \%)$ of the solar system inventories of many of the lighter elements (stable nuclei) by a SN II would produce large isotopic shifts that are not observed. The sole observed shift is in ${ }^{16} \mathrm{O}$ and is not correlated with any effects in other elements ( $\mathrm{Mg}, \mathrm{Ca}, \mathrm{Fe}, \mathrm{Si}$, etc.).

We conclude that a SN trigger to the formation of the solar system with injection of short-lived nuclei, particularly including ${ }^{26} \mathrm{Al}$ or ${ }^{41} \mathrm{Ca}$, is not an acceptable scenario. If we had used the Woosley \& Weaver [133] models the results would be essentially unchanged.

We note that the ${ }^{53} \mathrm{Mn}$ overproduction has led Meyer \& Clayton [198] to suggest that the source was a peculiar massive star that ejected only the external envelope rich in ${ }^{26} \mathrm{Al}$ and ${ }^{60} \mathrm{Fe}$ and not the innermost region rich in ${ }^{53} \mathrm{Mn}$. This ad hoc model is difficult to evaluate at the present time without further predictions based on the same idea. However, as ${ }^{60} \mathrm{Fe}$ is produced in layers external to the innermost regions producing ${ }^{53} \mathrm{Mn}$, increasing the mass cut (to reduce ${ }^{53} \mathrm{Mn}$ ) would leave, at least, the ${ }^{60} \mathrm{Fe}$ problem unresolved.

However, if we instead consider that a supernova source is responsible for the ${ }^{53} \mathrm{Mn}$ in the protosolar system, then the matter is greatly changed. Adopting this assumption, we have calculated the dilution factor to obtain ${ }^{53} \mathrm{Mn} /{ }^{55} \mathrm{Mn}$ $\sim 1 \times 10^{-4}$. This is in the range of the highest estimated values for the ESS [38]. In this case, $f_{0}=7 \times 10^{-6}$. The abundances of the other nuclei were calculated for this dilution factor (see last column, Table 2), keeping $\Delta_{1} \sim 1$ Myr as before. It is clear that this model is sufficient to also provide ${ }^{60} \mathrm{Fe}$ at the highest level currently considered in the ESS $\left({ }^{60} \mathrm{Fe} /{ }^{56} \mathrm{Fe} \geq 10^{-6},[46]\right)$. Then all 
of the other nuclei $\left({ }^{26} \mathrm{Al},{ }^{41} \mathrm{Ca},{ }^{107} \mathrm{Pd}\right)$ are essentially absent. If the ${ }^{53} \mathrm{Mn}$ in the ESS were decreased to $\left({ }^{53} \mathrm{Mn} /{ }^{55} \mathrm{Mn}\right) \sim 10^{-5}$ (which is compatible with many observations, see Section 11.2), then $f_{0} \sim 7 \times 10^{-7}$ and the ${ }^{60} \mathrm{Fe}$ would also approximately agree with an ESS value as currently proposed by Tachibana \& Huss [44] and Tachibana et al. [199], if one takes $\Delta_{2} \leq 1$ Myr. As there is no other stellar source for ${ }^{53} \mathrm{Mn}$, we conclude that a component of the solar inventory of short-lived nuclei must be from SN II; this only supplies the ${ }^{53} \mathrm{Mn}$ and some of the ${ }^{60} \mathrm{Fe}$, but no other short-lived nuclei. In considering both ${ }^{53} \mathrm{Mn}$ and ${ }^{60} \mathrm{Fe}$ we note that ${ }^{60} \mathrm{Fe}$ requires a stellar source and such a source can only be a supernova or an AGB star. A SNIa origin is possible for Fe group nuclei [200] [154]: this might include ${ }^{53} \mathrm{Mn}$, but probably not ${ }^{60} \mathrm{Fe}$, which derives from neutron captures.

We conclude that SNe II are excluded from further consideration for providing ${ }^{26} \mathrm{Al},{ }^{41} \mathrm{Ca},{ }^{107} \mathrm{Pd}$, etc. Instead, providing ${ }^{53} \mathrm{Mn}$ and ${ }^{60} \mathrm{Fe}$ from a SN II source is appealing, as the mass fraction that needs to be contributed to the ESS to account for them in a late addition is very small (with only small contributions to the other nuclei, e.g. $0.1 \%$ of the oxygen). If the SN II contribution ${ }^{53} \mathrm{Mn}$ and ${ }^{60} \mathrm{Fe}$ is from an earlier event (say $\Delta_{1} \sim 10^{7} \mathrm{yr}$ ), then the dilution factor would become significantly larger and there would be much less ${ }^{60} \mathrm{Fe}$. The general inventory of ${ }^{53} \mathrm{Mn}$ in the ISM should be quite sufficient to provide the plausible ESS values if the refreshment time by SNe II is less than $\sim 15$ Myr.

\section{What do AGB Stars Produce?}

A new generation of AGB yields for short-lived nuclei has been computed for this report. These calculations cover a range of stellar masses and metallicities. These new results are a substantial improvement over those discussed in Wasserburg et al. [97] and in Busso et al. [84]. The first results of these calculations were outlined by Busso et al. [58] and by Gallino et al. [201]. The new results are an improvement over the earlier calculations, as they include use of: i) cross sections from Bao et al. [202] plus several subsequent updates; ii) an improved technique for estimating the rate of bound-state electron captures on ${ }^{41} \mathrm{Ca}$ in the stellar envelope, obtained by averaging its lifetime over the distribution of temperature and density of the whole convective zone; and iii) the use of a finer grid for the basic stellar evolution models than used earlier. As discussed previously (Sec. 3) it is both impractical and unnecessary to perform all the network computations directly within complex and time-consuming stellar evolution codes. This is particularly important when one wants to build a very large database of stellar yields, for several different values of the stellar mass and metallicity. As far as rare species and neutron-capture products are concerned, post-process calculations remain a 
suitable tool, provided their input parameters can be estimated safely. Recently, Straniero et al. [89] computed a grid of stellar models with the explicit goal of generating reliable interpolation tools for deriving the basic detailed stellar models necessary to calculate nucleosynthesis in AGB stars. These rules apply to masses in the range 1 to $3 M_{\odot}$ and metallicities from $1 / 6 Z_{\odot}$ to $Z_{\odot}$.

Based on the rules recommended by Straniero [89], we prepared a finer network of models for calculating nucleosynthesis in AGB stars. All results discussed here are taken from these revised models. The complete stellar reference models were all calculated with the FRANEC evolutionary code. When this last and the MSSSP code [87] [88] [203] are used with the same treatment for mass loss and convective mixing, very similar results are obtained. This confirms the reliability of the evolutionary scenario. For details of the models and a complete review of stellar evolution preceding the late TP-AGB see Straniero et al. [83] in this volume. Mass loss was always simulated with the Reimers' parameterization, setting the values of the $\eta$ parameter to 0.3 (1.5 $M_{\odot}$ cases), $0.5\left(2.0 M_{\odot}\right), 1.5\left(3 M_{\odot}\right)$ and $5\left(5 M_{\odot}\right)$.

In all of the calculations presented here in Tables 3 to 5 for low and intermediate masses, the ${ }^{26} \mathrm{Al}$ contribution only takes into account its production in the $\mathrm{H}$ shell, its engulfment into the He-layers and burning through $(n, p)$ and $(n, \alpha)$ captures in the convective pulses, and subsequent free decay in the envelope (no CBP). The balance between these processes typically gives rise to envelope ratios ${ }^{26} \mathrm{Al} /{ }^{27} \mathrm{Al}$ of $\sim 5 \times 10^{-3}$ for low mass stars. When considering the net ${ }^{26} \mathrm{Al}$ inventory, this "base line" production should be added to the larger yields that may be derived from either CBP (low mass stars) or HBB (see Section 5 ). Note that our $5 M_{\odot}$ models do not include hot bottom burning in the envelope. For recent extensive calculations of HBB yields, especially for ${ }^{26} \mathrm{Al}$ and the ensuing effects on $\mathrm{Mg}$ isotopes, the reader is referred to Karakas and Lattanzio [123].

Concerning short-lived species produced by neutron captures, their yields depend on the $\left.{ }^{22} \mathrm{Ne}(\alpha, n)\right)^{25} \mathrm{Mg}$ reaction and the ${ }^{13} \mathrm{C}(\alpha, n){ }^{16} \mathrm{O}$ reaction (see Section 3). As this second process comes from a mixing mechanism of unknown nature that is treated parametrically, we present two sets of results: one considering only neutron captures in the convective pulses from ${ }^{22} \mathrm{Ne}$ burning, and one that includes both sources assuming a ${ }^{13} \mathrm{C}$ pocket as adopted in previous works (the 'standard' choice of [204] [84] [205]). This choice corresponds to $4 \times 10^{-6} M_{\odot}$ of ${ }^{13} \mathrm{C}$ in LMS and to $4 \times 10^{-7} M_{\odot}$ of ${ }^{13} \mathrm{C}$ in IMS (see Section 3 ). We note that the existence of a wide intrinsic spread of ${ }^{13} \mathrm{C}$ concentrations, from a few $10^{-6} M_{\odot}$ down to the complete absence of the ${ }^{13} \mathrm{C}$ pocket in the intershell zone, is demonstrated by observations of a wide range of $s$-process enhancements at a given metallicity in AGB stars [205] [206] [207]. This is also shown by the isotopic patterns in presolar grains (see e.g.[208]) and by $s$-process distributions in extremely metal poor stars [209]. The results for 
each stellar mass thus depend only on the amount of ${ }^{13} \mathrm{C}$ burned and on the metallicity.

We show here results for stars of $1.5,2,3$ and $5 M_{\odot}$, for both solar and $1 / 3$ solar metallicity. ${ }^{26} \mathrm{Al}$ is always produced in significant amounts, however less efficiently (by typically a factor of 3 ) than in $\mathrm{CBP}$ or in $\mathrm{HBB}$; its final yield then depends on the contributions from these processes.

As can be seen from equation 7 , the parameters that determine the abundances in the protosolar molecular cloud are the relative abundances, $\left(q_{E N V}^{I} / q_{0}^{I}\right)$, of each stable nuclide $I$ in the envelope relative to the ISM, and the ratio $\left(N^{R} / N^{I}\right)_{E N V}$ in the envelope. Note that in our earlier work [97] we gave $q_{\mathrm{He}}^{I} / q_{0}^{I}$ and $\left(N^{R} / N^{I}\right)_{\mathrm{He}}$ in the production shells. Here we explicitly calculated the values of $N^{R} / N^{I}$ and $q_{E N V}^{I} / q_{0}^{I}$ in the envelope over the evolution of the star as done in [58] [201]. The values of $q_{E N V}^{I} / q_{0}^{I}$ are presented in Table 3 and are shown in Fig. 6. As can be seen, for no ${ }^{13} \mathrm{C}$ pocket, for a given stellar mass and a given $Z$, the $q_{E N V}^{I} / q_{0}^{I}$ for all of the stable nuclei are close to $Z / Z_{\odot}$. However, with a ${ }^{13} \mathrm{C}$ pocket there is a substantial to great increase in $q_{E N V}^{I} / q_{0}^{I}$ for those elements of higher atomic number. This rule applies to all the cases shown, though they may differ from one another in other respects according to their ${ }^{13} \mathrm{C} / \mathrm{Fe}$ ratios (i.e., metallicity) and to their residual envelope masses (i.e., to the achieved dilution of C-rich and $s$-process-rich matter with unprocessed material).

The values of $\left(N^{R} / N^{I}\right)_{E N V}$ are given in Table 4 for the same models as above. It can be seen that, in general, for light nuclei $\left(N^{R} / N^{I}\right)_{E N V}$ is grossly the same with or without a ${ }^{13} \mathrm{C}$ pocket, while the $\left(N^{R} / N^{I}\right)_{E N V}$ of species heavier than iron increase for increasing neutron dose per seed nucleus. As a result, the presence of a ${ }^{13} \mathrm{C}$ pocket tends to give widely varying values of $\left(M_{0} / M_{E N V}\right) \alpha^{R, I}$ from one pair of nuclides to another. This makes it very difficult to reproduce isotopic ratios in heavy and light elements simultaneously, as it overproduces the heavier nuclei from neutron capture on the Fe seed. As a result, in seeking to explain a wide range of short-lived nuclei as well as ${ }^{107} \mathrm{Pd}$, we focus on AGB models with no ${ }^{13} \mathbf{C}$ pocket. Such stars can represent a substantial fraction of all AGB stars. The consequences of a standard ${ }^{13} \mathrm{C}$ pocket are discussed in Section 12: it can only provide some heavy nuclei.

\subsection{Determination of the dilution factor from ${ }^{107} \mathrm{Pd}$}

The value of $\left({ }^{107} \mathrm{Pd} /{ }^{108} \mathrm{Pd}\right)_{E S S}$ is well known since the discovery by Kelly \& Wasserburg [30]. Extensive work by Chen and Wasserburg [31][32] established internal isochrons for several iron meteorites of different classes with ${ }^{107} \mathrm{Pd} /{ }^{108} \mathrm{Pd}=2 \times 10^{-5}$ with a typically small range. The recent high-precision 
results by Carlson \& Hauri [33] confirm this abundance and we may consider the value as well-established. The very high value of ${ }^{107} \mathrm{Pd} /{ }^{108} \mathrm{Pd}$ reported by Carlson \& Hauri [33] for a small isotopic shift in ${ }^{107} \mathrm{Ag} /{ }^{109} \mathrm{Ag}$ on a bulk Allende sample was not confirmed by Woodland et al. [210]. As $\bar{\tau}_{107} \mathrm{Pd}=9.4 \times 10^{6}$ years, there will not be a major difference between the ESS value and the initial value at the time of injection into the ISM if $\Delta_{1}$ and $\Delta_{2} \leq 5 \times 10^{6}$ years. This short time scale for formation of some differentiated protoplanets is made clear by the discovery of ${ }^{26} \mathrm{Al}$ in basaltic achondrites (planetary crusts) as found by Srinivasan et al. [211]. Thus $\left({ }^{107} \mathrm{Pd} /{ }^{108} \mathrm{Pd}\right)_{E S S} \approx\left({ }^{107} \mathrm{Pd} /{ }^{108} \mathrm{Pd}\right)_{S C}$ and this value may be used to determine the dilution factor for a stellar model (see Sec. 7).

While ${ }^{107} \mathrm{Pd}$ can be produced in both $r$ - and $s$-processes, the solar inventory can not be attributed to a "standard" $r$-process source due to the long $\Delta_{1}$ implied by ${ }^{129} \mathrm{I}$. We therefore focus on an AGB source. Production of ${ }^{107} \mathrm{Pd}$ requires a single neutron capture on stable ${ }^{106} \mathrm{Pd}$ and thus occurs through both the ${ }^{22} \mathrm{Ne}(\alpha, \mathrm{n}){ }^{25} \mathrm{Mg}$ and the ${ }^{13} \mathrm{C}(\alpha, \mathrm{n}){ }^{16} \mathrm{O}$ neutron sources. The laboratory mean life of ${ }^{107} \mathrm{Pd}$ is not significantly changed in stellar interiors [212]. ${ }^{107} \mathrm{Pd}$ in turn captures a neutron to produce ${ }^{108} \mathrm{Pd}$ with production ratios roughly governed by the ratio of the $\left\langle\sigma^{i}\right\rangle$ values. Our results for ${ }^{107} \mathrm{Pd}$ (and its stable reference ${ }^{108} \mathrm{Pd}$ ) for an AGB source are shown in Tables 3 and 4 . The results for LMS with a ${ }^{13} \mathrm{C}$ pocket are far above those for no ${ }^{13} \mathrm{C}$. The choice of the magnitude of the ${ }^{13} \mathrm{C}$ pocket is very restricted in LMS by the fact that increased neutron capture for heavier nuclei greatly increases the $\mathrm{Pd}$ yield without changing the values for ${ }^{60} \mathrm{Fe},{ }^{41} \mathrm{Ca}$, or ${ }^{26} \mathrm{Al}$ (see Fig. 6). As a result, a standard ${ }^{13} \mathrm{C}$ pocket (sufficient to explain main solar $s$ abundances with LMS models) does not permit a simultaneous solution for any lower mass nuclide with those of higher masses (see [97] [84]). (The situation is different for an IMS, as the effects of the ${ }^{13} \mathrm{C}$-pocket are less important, cf. Fig. 6). A graph of the dilution factor $f_{0}$ versus $\left({ }^{107} \mathrm{Pd} /{ }^{108} \mathrm{Pd}\right)_{0}$ (the subscript refers to time since injection), for the case of no pocket, is shown in Fig. 7, allowing for a range of values of the time delay $\Delta_{2}$. For no ${ }^{13} \mathrm{C}$ pocket and $Z=Z_{\odot} / 3$ to $Z_{\odot}$, models predict a restricted range of ${ }^{107} \mathrm{Pd} /{ }^{108} \mathrm{Pd}$ in the envelope for low mass stars up to $3 M_{\odot}$, while a much larger yield is predicted for a $5 M_{\odot}$ model. For the case of $f_{0} \simeq 10^{-2.3}$ and $Z=Z_{\odot}$ we find $\left({ }^{107} \mathrm{Pd} /{ }^{108} \mathrm{Pd}\right)_{0} \simeq 3 \times 10^{-5}$. This is more than sufficient to account for the ${ }^{107} \mathrm{Pd}$ found in the Early Solar System. It can be seen that for low mass stars with no ${ }^{13} \mathrm{C}$ pocket the possible range of $f_{0}$ values is $2 \times 10^{-3} \leq f_{0} \leq 1.5 \times 10^{-2}$. For a $5 M_{\odot}$ model the range is $2 \times 10^{-4}<f_{0}<4 \times 10^{-4}$. These ranges of $f_{0}$ will now be used to estimate the contributions for all other nuclei produced by AGB models. 


\subsection{Calculated Abundances from an $A G B$ source}

Using the composition of the winds for different AGB stellar models and the dilution factor obtained from ${ }^{107} \mathrm{Pd}$, we may now calculate the abundances of short-lived nuclei to be expected at a time $\Delta_{1}$ in a mix of AGB ejecta and ISM material. Examples of these results are shown in Tables 5 and 6 . Table 5 refers to the solar metallicity models using the range of dilution factors shown in Fig. 7. Similarly, the range of $f_{0}$ found from ${ }^{107} \mathrm{Pd}$ is superimposed on the ${ }^{60} \mathrm{Fe} /{ }^{56} \mathrm{Fe}$ versus $\mathrm{f}_{0}$ graph in Fig. 8 to show the range of ${ }^{60} \mathrm{Fe} /{ }^{56} \mathrm{Fe}$ corresponding to these bounds. A value of $f_{0}$ from its permitted range can then be combined with $\left({ }^{41} \mathrm{Ca} /{ }^{40} \mathrm{Ca}\right)_{E S S}$ to determine $\Delta_{1}$ and match the ESS inventory for this very short-lived nuclide. The corresponding values for all the other isotopes are then presented at that $f_{0}$ and $\Delta_{1}$, along with the $\Delta_{2}$ for an exact match to ${ }^{107} \mathrm{Pd}$, in Tables 5 and 6 . These can be compared with the ESS values reported in Table 1.

From inspection of Table 5, we find the following general rules:

i) LMS models (1.5 or $2 M_{\odot}$ ) can be a suitable source for ${ }^{41} \mathrm{Ca}$ and ${ }^{107} \mathrm{Pd}$ and can also predict a substantial inventory of all other species listed;

ii) It can again be seen that the typical values of $\left({ }^{26} \mathrm{Al} /{ }^{27} \mathrm{Al}\right)_{E N V} \sim(3-5) \times$ $10^{-3}$, which derive from pure $\mathrm{H}$-shell burning without $\mathrm{CBP}$, are insufficient to match the ESS values by a factor of $\sim 3$;

iii) ${ }^{60} \mathrm{Fe}$ can be accounted for by a late addition from a low mass AGB only at the lowest of the recently suggested ESS ratios;

iv) IMS models can easily produce abundant ${ }^{60} \mathrm{Fe}$, sufficient to account for almost the highest $\left({ }^{60} \mathrm{Fe} /{ }^{56} \mathrm{Fe}\right)_{E S S}$ that has been proposed; they also copiously produce other isotopes. However, as shown for a $5 M_{\odot}$ star, the value of $\Delta_{1}$ required by ${ }^{41} \mathrm{Ca}$ is very short.

Both standard (i.e. without CBP) LMS and IMS models end up with the requirements that other mechanisms or sources must supplement AGB production for ${ }^{26} \mathrm{Al}$ and one or another isotope of the short list presented in Tables 3 to 5. As indicated earlier [61], CBP produces large amounts of ${ }^{26} \mathrm{Al}$. For ${ }^{26} \mathrm{Al}$ we have calculated the $\left({ }^{26} \mathrm{Al} /{ }^{27} \mathrm{Al}\right)_{E N V}$ required in order to obtain $\left({ }^{26} \mathrm{Al} /{ }^{27} \mathrm{Al}\right)_{E S S}=5 \times 10^{-5}$. The exact envelope value required to provide a solution is indicated in parentheses for each case in Table 5 . These levels are well within the reach of CBP models without stretching to high $T_{P}$ values.

We thus consider the ${ }^{26} \mathrm{Al}$ observations to be explained by an AGB star with some CBP. With regard to possible matches with ESS values, we note that a compromise exists between the two cases $(1.5-2) M_{\odot}$ and $5 M_{\odot}$. The model of 
a $3 M_{\odot}$ star provides the best balance and accounts for the largest number of ESS radioactivities, including ${ }^{41} \mathrm{Ca}$ and ${ }^{60} \mathrm{Fe}$, with reasonable values of the time delays. As an example of this, we show in Table 6 the result of applying the above approach to a $3 M_{\odot}, Z=Z_{\odot} / 3$ case. As can be seen, a late contamination by such a star could explain the ESS abundances of ${ }^{26} \mathrm{Al}$, ${ }^{41} \mathrm{Ca},{ }^{107} \mathrm{Pd}$, and ${ }^{60} \mathrm{Fe}$, (even for quite high ESS values). It would also give substantial contributions of ${ }^{135} \mathrm{Cs}$. The matter of ${ }^{36} \mathrm{Cl}$ is discussed in Section 11.5 devoted to that isotope.

All the computations of Tables 5 and 6 predict upper bounds for ${ }^{205} \mathrm{~Pb}$. This last datum cannot be better determined, due to the high uncertainty of ${ }^{205} \mathrm{~Pb}$ survival after a thermal pulse (see Mowlavi et al. [213] for a discussion). As in our original calculations [97], we note that a large fraction of this ${ }^{205} \mathrm{~Pb}$ decays before being ejected, at least in LMS cases. Due to the complex behavior of the couple ${ }^{205} \mathrm{~Pb}^{205} \mathrm{Tl}$ in He-shell conditions, the higher temperature of IMS models (including that of Table 6) would favor the survival of ${ }^{205} \mathrm{~Pb}$ in the critical interval after a pulse (a few hundred years) before it is transferred to the envelope by dredge up. Hence IMS models are probably the most promising site for ${ }^{205} \mathrm{~Pb}$ formation. Thorough and detailed calculations are required to obtain precise estimates of the rates for the weak interactions involved.

\section{Comments on some critical isotopes}

The overall results presented above give a reliable and broad assessment of what different sources could provide. There are important, critical issues involving the abundances of certain nuclei inferred from measurements in meteorites. In some cases there are uncertainties in the network calculations. In this section we present some considerations of the reliability or uncertainty of the results used above.

\subsection{Estimating the ${ }^{60} \mathrm{Fe}$ inventory}

The isotope ${ }^{60} \mathrm{Fe}$ plays a key role in determining $\Delta_{1}$ for ${ }^{53} \mathrm{Mn}$ and ${ }^{60} \mathrm{Fe}$. Both are produced in abundance by SNe II and are present in high levels in the ISM for the UP model. As ${ }^{60} \mathrm{Fe}$ (but not ${ }^{53} \mathrm{Mn}$ ) is also produced by AGB stars, they would add to the inventory from SNe II. Because of its importance, we present a short review of the history of the searches for ${ }^{60} \mathrm{Fe}$.

The first hint of ${ }^{60} \mathrm{Fe}$ in the solar system was found as excesses of ${ }^{60} \mathrm{Ni}$ in CAIs [214]. These data can be interpreted to indicate that the ratio at the time of some CAI formation was ${ }^{60} \mathrm{Fe} /{ }^{56} \mathrm{Fe}=(1.6 \pm 0.5) \times 10^{-4}$. However, the measured 
${ }^{60} \mathrm{Ni}$ excesses might reflect isotopically anomalous $\mathrm{Ni}$ from pre-existing stellar condensates rather than decay of ${ }^{60} \mathrm{Fe}$ [42]. Further, it was found by Völkening \& Papanastassiou [215] that some CAIs contained anomalous Fe with conspicuous excesses of ${ }^{58} \mathrm{Fe}$. Thus the straightforward interpretation of the CAI data remains unclear. The first clear evidence indicating ${ }^{60} \mathrm{Fe}$ was found in eucrites [42] [43], which are basaltic rocks. This study was well-designed as eucrites are greatly depleted in $\mathrm{Ni}$ from the formation of an $\mathrm{Fe} / \mathrm{Ni}$ core in their parent planetesimal. However, the data showed no good correlation for ${ }^{60} \mathrm{Ni}^{*}$ versus ${ }^{56} \mathrm{Fe}$. The inferred ${ }^{60} \mathrm{Fe} /{ }^{56} \mathrm{Fe}$ for eucrites was far lower than that inferred for CAIs and differs considerably from one eucrite to another: $3.9 \times 10^{-9}$ to $4.3 \times 10^{-10}$. In contrast, the abundances of ${ }^{53} \mathrm{Mn}(\bar{\tau}=5.3 \mathrm{Myr})$ inferred for these same eucrites were typically uniform $\left({ }^{53} \mathrm{Mn} /{ }^{55} \mathrm{Mn} \approx 1-4 \times 10^{-6}\right)$ [41]. Even assuming a value of ${ }^{60} \mathrm{Fe} /{ }^{56} \mathrm{Fe}$ for the eucrites, there remained the question of $\Delta_{2}$ for formation/metamorphism of these basaltic rocks. As $\bar{\tau}_{60} \mathrm{Fe}=2.2 \times 10^{6} \mathrm{yr}$, if $\Delta_{2}=5 \times 10^{6} \mathrm{yr}$, the initial value would then be ten times greater than for the eucrite.

Several searches for evidence of ${ }^{60} \mathrm{Fe}$ were made in chondrites, which did not totally melt and are far more primitive than eucrites [43] [216] [217]. Troilite (FeS) from a relatively metamorphosed chondrite, Ste. Marguerite, gave an upper limit of $2.4 \times 10^{-8}$ for ${ }^{60} \mathrm{Fe} /{ }^{56} \mathrm{Fe}$ [43]. Ion microprobe studies of unmetamorphosed olivine phenocrysts in chondrules (a major constituent of chondrites) gave no resolvable ${ }^{60} \mathrm{Ni}^{*}$ in unequilibrated ordinary chondrites [217] [74]. The upper limit for ${ }^{60} \mathrm{Fe} /{ }^{56} \mathrm{Fe}$ in a Semarkona chondrule was $3.4 \times 10^{-7}$ [217], while that for Bishunpur and Semarkona chondrules was $1.6 \times 10^{-6}[74]$. Measurements of a sulfide-rich opaque inclusion and spinels within a CAI [74] gave the upper limit on ${ }^{60} \mathrm{Fe} /{ }^{56} \mathrm{Fe}$ of $1.7 \times 10^{-6}$. Very recently, there have been important observations in the search for ${ }^{60} \mathrm{Fe}$ that show clear correlations of ${ }^{60} \mathrm{Ni} /{ }^{61} \mathrm{Ni}$ versus ${ }^{56} \mathrm{Fe} /{ }^{61} \mathrm{Ni}$ with very high values of $\mathrm{Fe} / \mathrm{Ni}\left(\sim 10^{5}-10^{6}\right)[44]$ [218] [219]. The results of [44] and [199] gave ${ }^{60} \mathrm{Fe} /{ }^{56} \mathrm{Fe} \simeq 1 \times 10^{-7}$ for several troilite samples. The results by Mostefaoui et al. [219] on troilites gave $\left({ }^{60} \mathrm{Fe} /{ }^{56} \mathrm{Fe}\right)_{0} \simeq 1 \times 10^{-6}$. Most recently, measurements by Tachibana et al. [199] on silicates in chondrules of very low metamorphic grade chondrites gave ${ }^{60} \mathrm{Fe} /{ }^{56} \mathrm{Fe} \simeq 2 \times 10^{-7}$. If $\Delta_{2}=2 \times 10^{6} \mathrm{yr}$, this implies $\left({ }^{60} \mathrm{Fe} /{ }^{56} \mathrm{Fe}\right)_{0}=5 \times 10^{-7}$. This matter is under intense study by several groups at present. As noted in [58], the very high ${ }^{60} \mathrm{Fe}$ abundances now indicated should readily permit high precision isochrons to be established using TIMS or ICPMS techniques with only small Fe-Ni fractionation between phases.

Fig. 8 shows a plot of the dilution factor $f_{0}$ versus the ratio $\left({ }^{60} \mathrm{Fe} /{ }^{56} \mathrm{Fe}\right)_{0}$ in a cloud in which the ejecta from an AGB star (see previous section) were instantaneously mixed. Assuming a dilution factor of $f_{0}=10^{-2}$ we find that the values of $\left({ }^{60} \mathrm{Fe} /{ }^{56} \mathrm{Fe}\right)_{0}$ range from $10^{-7}$ to $4 \times 10^{-7}$ for $M<3 M_{\odot}$ and $Z=Z_{\odot}$. The acceptable ranges of $f_{0}$ as determined by ${ }^{107} \mathrm{Pd}$ are shown as shaded areas on the graph of $f_{0}$ versus ${ }^{60} \mathrm{Fe} /{ }^{56} \mathrm{Fe}$. For a model of $5 M_{\odot}$ and $Z_{\odot}$, 
$\left({ }^{60} \mathrm{Fe} /{ }^{56} \mathrm{Fe}\right)_{0}$ values in excess of $10^{-6}$ would be permitted with $f_{0}$ in the range allowed by ${ }^{107} \mathrm{Pd}$. There is thus a wide range of $\left({ }^{60} \mathrm{Fe} /{ }^{56} \mathrm{Fe}\right)_{0}$ possible (more than an order of magnitude) from AGB stars with solar abundances at low dilutions. The repeated statements in the literature that ${ }^{60} \mathrm{Fe}$ is a clear mark of SNe is incorrect as shown long ago. It is evident that if $\left({ }^{60} \mathrm{Fe} /{ }^{56} \mathrm{Fe}\right)_{E S S}$ lies between $2 \times 10^{-6}$ and $2 \times 10^{-7}$, then a $1.5 M_{\odot}$ case is excluded. The possible AGB sources are then restricted to $2-3 M_{\odot}$.

Note that in earlier efforts to determine possible AGB contributions, Wasserburg et al. [97] focused on a $1.5 M_{\odot}$ source. It was found that the local ratio in the production zone was ${ }^{60} \mathrm{Fe} /{ }^{56} \mathrm{Fe}=10^{-4}$ to $10^{-3}$ [97]. This gave fully adequate ${ }^{60} \mathrm{Fe}$ to provide the inventory then current of $\left({ }^{60} \mathrm{Fe} /{ }^{56} \mathrm{Fe}\right)_{\Delta_{2}} \sim 10^{-8}$ with reasonable choices of $\Delta_{1} \lesssim 10^{7}$ yr. Those calculations are still applicable for a $1.5 M_{\odot}$ source with only minor changes for the prediction of other short-lived nuclei.

From the existing data sets, we now must consider a rather wide range of much higher values of ${ }^{60} \mathrm{Fe}$ abundance in our models. For the purposes considered here, we will consider the range $2 \times 10^{-7}$ to $2 \times 10^{-6}$, as shown in Table 1 .

With the improved models discussed in Section 3 and for no ${ }^{13} \mathrm{C}$ pocket at solar metallicities, there is a very restricted range of ${ }^{60} \mathrm{Fe} /{ }^{56} \mathrm{Fe}$ in the envelope for $M \leq 3 M_{\odot}$ (see Tables 3 to 5 ). It follows that for $M \lesssim 3 M_{\odot}$ and $Z_{\odot}$, the $\left({ }^{60} \mathrm{Fe} /{ }^{56} \mathrm{Fe}\right)_{0}$ is then essentially fixed by the dilution factor (see equation 7 ). On the other hand, there is a large increase in ${ }^{60} \mathrm{Fe}$ production at $5 M_{\odot}$ due to the greatly increased temperatures of the He shell. For this case note that $q_{E N V}$ also drops with decreasing $Z$ due to consumption of the Fe seed.

We now explore how robust these results are in terms of the nuclear physics. A major uncertainty in the ${ }^{60} \mathrm{Fe}$ production lies in the neutron capture cross sections of both ${ }^{59} \mathrm{Fe}$ and ${ }^{60} \mathrm{Fe}$, which are based on theoretical estimates only. The Maxwellian-averaged cross section of ${ }^{60} \mathrm{Fe}$ at $30 \mathrm{keV}$ is rather low: $\langle\sigma\rangle(30$ $\mathrm{keV})\left({ }^{60} \mathrm{Fe}\right)=3.65$ mbarn, according to Woosley et al. [220]. Consequently, only a small fraction of the ${ }^{60} \mathrm{Fe}$ produced can undergo further neutron captures. On the other hand, ${ }^{60} \mathrm{Fe}$ synthesis directly depends on the cross section of ${ }^{59} \mathrm{Fe}$, whose value at $30 \mathrm{keV}$, as estimated by Woosley et al. [220] is 12.3 mbarn. More recent calculations, based on a different theoretical approach, have been performed by Rauscher \& Thielemann [221]; they obtain $\langle\sigma\rangle(30$ $\mathrm{keV})\left({ }^{59} \mathrm{Fe}\right)=22.5$ mbarn. We adopt here these recent estimates, but we verified that, if we had instead used the old cross sections for both ${ }^{59} \mathrm{Fe}$ and ${ }^{60} \mathrm{Fe}$, our production of ${ }^{60} \mathrm{Fe}$ (as given in Tables 3, 4) would have been reduced by a factor $\approx 1.8$ for all the $M=1.5$ and $3 M_{\odot}$ models, and by a factor of $\sim 1.2$ for the $M=5 M_{\odot}$ models. We conclude that variations of the neutron capture cross sections cannot change our predictions by more than a factor of 2 . 


\section{2 ${ }^{53} \mathrm{Mn}$}

The nuclide ${ }^{53} \mathrm{Mn}$ is particularly important since it can only be produced in $\mathrm{SNe}$ or by proton bombardment. It was first shown by Birck \& Allègre [38] [39] that excess ${ }^{53} \mathrm{Cr}$ was present in CAIs. While there appears to be a correlation of ${ }^{53} \mathrm{Cr}^{*}$ with $\mathrm{Mn}$ in CAIs, there is in addition a widespread occurrence of variations in ${ }^{54} \mathrm{Cr}$ which can only be ascribed to endemic, that is widespread, nuclear effects in meteorites due to incomplete mixing from different sources [222]. As recognized by Birck \& Allègre [38], it is not obvious that the ${ }^{53} \mathrm{Cr}^{*}$ can be unambiguously attributed to in situ decay of ${ }^{53} \mathrm{Mn}$. A recent report by Papanastassiou et al. [223] on a CAI known to contain ${ }^{26} \mathrm{Al}$ showed with precise data a correlation of ${ }^{53} \mathrm{Cr}^{*}$ with $\mathrm{Mn} / \mathrm{Cr}$ giving ${ }^{53} \mathrm{Mn} /{ }^{55} \mathrm{Mn}$ $=1.5 \times 10^{-4}$, in agreement with Birck \& Allègre [38]. This sample also showed large excesses of ${ }^{54} \mathrm{Cr} /{ }^{52} \mathrm{Cr}$. We consider this value as the upper estimate of ${ }^{53} \mathrm{Mn} /{ }^{55} \mathrm{Mn}$ in the ESS. In contrast, from the well-defined study of Birck \& Allègre [39] on a pallasite (stony-iron), it is clear that ${ }^{53} \mathrm{Mn}$ was present in differentiated planets at a level of ${ }^{53} \mathrm{Mn} /{ }^{55} \mathrm{Mn}=2.3 \times 10^{-6}$. Extensive studies of the ${ }^{53} \mathrm{Mn}^{53} \mathrm{Cr}$ system in eucrites (basaltic achondrites), which are planetary differentiates, show very well-defined correlations of ${ }^{53} \mathrm{Cr} /{ }^{52} \mathrm{Cr}$ versus ${ }^{55} \mathrm{Mn} /{ }^{52} \mathrm{Cr}$. This work demonstrates the presence of ${ }^{53} \mathrm{Mn}$ in this stage of planetary evolution and yields $\left({ }^{53} \mathrm{Mn} /{ }^{55} \mathrm{Mn}\right)_{\Delta_{2}}=(4.7 \pm 0.5) \times 10^{-6}$ for some meteorites. In some cases it was possible to determine internal isochrons with slopes corresponding to about the same value (see the extensive report by Lugmair \& Shukolyukov [41]). Studies of whole-rock chondrites with very limited fractionation of $\mathrm{Mn}$ from $\mathrm{Cr}$ indicate ${ }^{53} \mathrm{Mn} /{ }^{55} \mathrm{Mn} \sim 9 \times 10^{-6}$ [224] [41]. We take this value as a reasonably sound estimate of the ESS value, although somewhat higher values have been found in late-stage alteration products in a CM2 chondrite [225]. The issue of the times $\Delta_{2}$ at which the eucrites formed and the problem of thermal metamorphism persist. This is well recognized by these authors and is evident in the eucrite samples where ${ }^{53} \mathrm{Mn}$ is essentially dead (cf. the meteorite Caldera, [226]). The reader is directed to the extensive reviews by McKeegan \& Davis [57], and Lugmair \& Shukolyukov [41]. In our discussion we will consider two values of $\left({ }^{53} \mathrm{Mn} /{ }^{55} \mathrm{Mn}\right)_{\Delta_{1}}$, specifically $10^{-5}$ and $10^{-4}$ (roughly covering the range of data shown in Table 1 ). The higher value corresponds to the high value in CAIs and is uncertain due to endemic nuclear effects in CAIs. The lower value is somewhat above the highest value found in planetary differentiates.

\section{3 ${ }^{26} \mathrm{Al}$}

The decay of ${ }^{26} \mathrm{Al}(\bar{\tau}=1.03 \mathrm{Myr})$ to ${ }^{26} \mathrm{Mg}$ has a special role. The discovery of ${ }^{26} \mathrm{Mg}^{*}$ correlated with ${ }^{27} \mathrm{Al}$ in the CAIs from the Allende CV3 meteorite 
showed that a nuclide with the chemistry of $\mathrm{Al}$ was present in the early solar system with an abundance of ${ }^{26} \mathrm{Al} /{ }^{27} \mathrm{Al}=5 \times 10^{-5}$ (see Fig. 9). This is analogous to the ${ }^{129} \mathrm{I}$ case discussed earlier, but because of the short mean life of this nuclide, a rather immediate synthesis of ${ }^{26} \mathrm{Al}$ and rapid formation of the solar system is required. The discovery of ${ }^{26} \mathrm{Al}$ changed the time scales from $\sim 10^{2}$ Myr to $\sim 1 \mathrm{Myr}$. These results were followed by studies that found evidence of ${ }^{26} \mathrm{Al}$ in other $\mathrm{CV}$ meteorites [227] with abundant CAIs. A considerable number of analyses on Allende and other CV meteorites showed that the evidence for ${ }^{26} \mathrm{Al}$ in CAIs was widespread, with high values of ${ }^{26} \mathrm{Al} /{ }^{27} \mathrm{Al} \sim 5 \times 10^{-5}$ and many lower values. The results compiled by MacPherson et al. [228] showed that these observations are a general rule. The search for CAIs in different chondrite classes showed that these lithic types were almost universally present albeit in very small amounts. There was also clear evidence of some live ${ }^{26} \mathrm{Al}[229]$ in these CAIs in typical chondrites. It has now been shown that CAIs in chondrites ranging from unequilibrated to equilibrated (i.e., varying degrees of thermal metamorphism to permit element diffusion and some recrystallization), all show varying degrees of ${ }^{26} \mathrm{Al} /{ }^{27} \mathrm{Al}$ (cf. [230] [231]). It follows that CAIs containing ${ }^{26} \mathrm{Al}$ with a maximum value of ${ }^{26} \mathrm{Al} /{ }^{27} \mathrm{Al} \sim 5 \times 10^{-5}$ are present in all chondritic meteorite classes.

The broader issue is how widespread the evidence is for ${ }^{26} \mathrm{Al}$ in the bulk material in the chondritic meteorites that essentially represent the relative solar abundances of condensible elements. The most abundant lithic components of chondrites are the spherical-subspherical chondrules which are formed by crystallization of liquid droplets of silicates.

The first definitive evidence for ${ }^{26} \mathrm{Al}$ in a chondrule was discovered by Hutcheon $\&$ Hutchison [232]. These workers found a clear excess of ${ }^{26} \mathrm{Mg}$ well correlated with $\mathrm{Al} / \mathrm{Mg}$ and yielding ${ }^{26} \mathrm{Al} /{ }^{27} \mathrm{Al} \approx 8 \times 10^{-6}$. This ratio is a factor of 6 below the typical high values found in CAIs. Intensive studies by other workers established that some chondrules have evidence of ${ }^{26} \mathrm{Al}$ but almost always in the range $5 \times 10^{-6}$ to $10^{-6}$. In most chondrules ${ }^{26} \mathrm{Mg}^{*}$ was not detectable at the precision available (cf. [233][234] [217], see also discussion and Fig. 3 in [57]). The general rule was that chondrules were formed with much less ${ }^{26} \mathrm{Al}$ than typical CAIs. If the ${ }^{26} \mathrm{Al} /{ }^{27} \mathrm{Al}$ ratios are interpreted as due to a time difference, this would require that most chondrules were formed or metamorphosed 1-5 Myr later than CAIs. We note that the low ${ }^{26} \mathrm{Al}$ found by Russell et al. [234] is in a sodium-rich glass in a chondrule. This chondrule clearly did not form under "nebular" conditions and, as $\mathrm{Na}$ is very volatile, required a very high $\mathrm{Na}$ partial pressure in the gas phase to condense silicate droplets with such a high $\mathrm{Na}$ concentration. It is also evident that there are CAIs with low or no ${ }^{26} \mathrm{Al}$. The issue is whether the lower values represent radioactive decay with the passage of time or if they represent the range of ${ }^{26} \mathrm{Al}$ available from a state that was highly heterogeneous with some materials that were formed without ${ }^{26} \mathrm{Al}$. The mechanisms for producing CAIs are then an issue. If one assumes 
a homogeneous initial state, then it follows that CAIs can be formed at later times. This is, of course, possible. If the systems were highly heterogeneous (i.e., no well-defined initial state) then there is the problem of explaining the rather sharp cut-off and peak at ${ }^{26} \mathrm{Al} /{ }^{27} \mathrm{Al} \approx 5 \times 10^{-5}$.

With significant improvements in the precision of $\mathrm{Mg}$ isotopic measurements using MC-ICPMS techniques, it has become possible to measure small $\mathrm{Mg}$ isotopic fractionation effects and to measure ${ }^{26} \mathrm{Mg}^{*}$ in bulk chondrules with low ${ }^{27} \mathrm{Al} /{ }^{24} \mathrm{Mg}$ [235]. These workers found evidence for ${ }^{26} \mathrm{Al}$ in several chondrules. A recent report by Bizzarro et al. [236] found that ${ }^{26} \mathrm{Al}$ was present in chondrules (in addition to CAIs) with ${ }^{26} \mathrm{Al} /{ }^{27} \mathrm{Al}$ ranging from $5.7 \times 10^{-5}$ to $1.4 \times 10^{-5}$. These are all important results and require considerable care in verification; they have not been substantiated and may be subject to serious revision. Nonetheless, there is now abundant evidence for the presence of ${ }^{26} \mathrm{Al}$ in broadly distributed chondritic material. In planetary differentiates, evidence for ${ }^{26} \mathrm{Al}$ was first found by Srinivasan et al. [211]. This report was confirmed [237]. However, the ${ }^{26} \mathrm{Al} /{ }^{27} \mathrm{Al}$ in this planetary differentiate is uncertain because ${ }^{26} \mathrm{Mg}^{*}$ was enriched compared to ${ }^{24} \mathrm{Mg}$ in the plagioclases, but was essentially uniform (i.e. independent of the $\mathrm{Al} / \mathrm{Mg}$ ratio) while the pyroxene shows normal ${ }^{26} \mathrm{Mg} /{ }^{24} \mathrm{Mg}$. This system appears to be isotopically homogenized within the plagioclase and indicates low temperature metamorphism resulting in partial isotopic equilibration. Presence of ${ }^{26} \mathrm{Al}$ would not be detectable in the Mg-rich phases.

A most important study of a eucrite by Nyquist et al. [238] reports an isochron with ${ }^{53} \mathrm{Mn} /{ }^{55} \mathrm{Mn}=(4.6 \pm 1.7) \times 10^{-6}$ and also an ${ }^{26} \mathrm{Al}$ isochron with ${ }^{26} \mathrm{Al} /{ }^{27} \mathrm{Al}$ $=(1.18 \pm 0.14) \times 10^{-6}$. A more recent report by Wadhwa et al. [239] using multicollector ICPMS techniques has confirmed these results with much higher precision. It also provided a ${ }^{207} \mathrm{~Pb} /{ }^{206} \mathrm{~Pb}$ age of $(4565.03 \pm 0.85) \mathrm{Myr}$, improved in precision due to using the computer printout. These results unambiguously demonstrate the presence of two short-lived nuclei in an ancient basaltic meteorite in self-consistent amounts. Thus, there is the general rule that both ${ }^{26} \mathrm{Al}$ and ${ }^{53} \mathrm{Mn}$ were present at appropriate abundances in early planets. There must also have been ${ }^{60} \mathrm{Fe}$ present. These are the heat sources required for melting of protoplanets [15] and must have been widely and generally distributed through the solar system.

In general, ${ }^{26} \mathrm{Al}$ appears to have been present in CAIs, chondrules, and planetary differentiates. Thus, it was widespread throughout condensed matter in the solar system at different stages. The ranges in ${ }^{26} \mathrm{Al} /{ }^{27} \mathrm{Al}$ may reflect differences in time or an initial heterogeneous distribution. If the source of ${ }^{26} \mathrm{Al}$ is local (i.e., within the solar system), then it must provide this nuclide to the bulk of materials forming "condensed" planetary matter. If the source is an external one (e.g., an AGB star), then it was rather well mixed with the material that formed solids. 
The peak in the observed distribution of ${ }^{26} \mathrm{Al} /{ }^{27} \mathrm{Al}=5 \times 10^{-5}$ is called the "canonical value". The distribution below this peak is in general easily attributable to redistribution of $\mathrm{Mg}$ as a result of metamorphism, but some unmetamorphosed CAIs having almost no ${ }^{26} \mathrm{Al}$ (e.g. HAL) do exist. The latter observation requires that some CAIs formed quite late, or that the distribution of ${ }^{26} \mathrm{Al}$ was very heterogeneous, with a sharp high peak. Also this well-defined and highly populated peak shows a range: this could be due to metamorphism from an initial state that had a uniform and fixed value of ${ }^{26} \mathrm{Al} /{ }^{27} \mathrm{Al}$. However, a study by El Goresy et al. [240] showed that one unaltered CAI was made up of two petrographically distinct CAI units that were later entrapped in a third liquid of CAI composition. Isotopic studies of these lithic subunits showed that they had clearly distinguishable ${ }^{26} \mathrm{Al} /{ }^{27} \mathrm{Al}$ ratios $(3.3$ to 6$) \times 10^{-5}$ that correlated with the sequence defined petrographically [241]. If they started with the same ${ }^{26} \mathrm{Al} /{ }^{27} \mathrm{Al}$ initial value, then this requires that these three units (in the same droplet) formed over a time interval of $\approx 4 \times 10^{5} \mathrm{yr}$. Alternatively, they could very possibly represent multiple droplet formation in a single event, but from a starting material that was heterogeneous. Recent results by Galy, Hutcheon \& Grossman [242], by Liu, Iizuka \& McKeegan [243] and by Young et al. [244] using "bulk" CAI samples in a new generation of high-precision measurements show that the spread in ${ }^{26} \mathrm{Al} /{ }^{27} \mathrm{Al}$ is rather general, with some samples having a "supra-canonical" value of ${ }^{26} \mathrm{Al} /{ }^{27} \mathrm{Al} \approx 6 \times 10^{-5}$. These more recent studies point to a higher value of ${ }^{26} \mathrm{Al} /{ }^{27} \mathrm{Al}$ in the ESS. However, there are no data indicating ${ }^{26} \mathrm{Al} /{ }^{27} \mathrm{Al}$ ratios that are a factor of two or more higher than the canonical value.

With regard to measurements on "bulk" inclusions, these results are like "total rock" ages and require considerable attention. Shifts in the initial ${ }^{26} \mathrm{Mg} /{ }^{24} \mathrm{Mg}$ of $0.02 \%$ would cause $10-20 \%$ shifts in the estimated ${ }^{26} \mathrm{Al} /{ }^{27} \mathrm{Al}$. The argument that the "total" systems are closed is not readily defensible. The reported effects appear to be real and suggest that variations in the initial ${ }^{26} \mathrm{Mg} /{ }^{24} \mathrm{Mg}$ should be found in careful studies of internal isochrons. There is certainly evidence of "subnormal" ${ }^{26} \mathrm{Mg} /{ }^{24} \mathrm{Mg}(\sim 1$ per mil) in pyroxenes in some Type B CAIs as found using TIMS techniques. These effects are an order of magnitude greater than from the ${ }^{26} \mathrm{Al}$ inventory (see Fig. 10 in [128]). A re-examination of CAI phases appears to be in order using improved techniques and considering the presence of nuclear anomalies.

The question remains as to whether this range in ${ }^{26} \mathrm{Al} /{ }^{27} \mathrm{Al}$ is due to somewhat incomplete mixing of presolar stellar debris (a small range in the dilution factor), or if the ${ }^{26} \mathrm{Al}$ is produced by an extremely intense irradiation, with some small local differences in the shielding and thorough mixing, but not producing "hot spots."

In our discussion we treated the ${ }^{26} \mathrm{Al}$ problem as if the bulk solar system initially had $\left({ }^{26} \mathrm{Al} /{ }^{27} \mathrm{Al}\right)_{\odot} \cong 5 \times 10^{-5}$. A small local variation in the dilution 
factor (e.g. incomplete mixing) could readily account for small deviations. The range of possible shifts to higher values mentioned above will not greatly affect the arguments given here. The fundamental question is: do the samples represent the bulk solar value or, do they represent only solids and materials in parts of the accretion disc?

\section{$11.4{ }^{41} \mathrm{Ca}$}

The first hint of ${ }^{41} \mathrm{Ca}$ was found by Hutcheon et al. [245], who could only establish an upper bound of ${ }^{41} \mathrm{Ca} /{ }^{40} \mathrm{Ca} \leq(8 \pm 3) \times 10^{-9}$ in CAIs. Clear evidence for the presence and abundance of ${ }^{41} \mathrm{Ca}$ in the ESS was found in CAIs by Srinivasan et al. [48] [49]. Further evidence was found by Sahijpal et al. [246] and a good correlation line was found for hibonite crystals in CAIs from Murchison, Allende and Efremovka. These results showed ${ }^{41} \mathrm{Ca} /{ }^{40} \mathrm{Ca}=1.4 \times 10^{-8}$ for CAIs that had ${ }^{26} \mathrm{Al} /{ }^{27} \mathrm{Al} \cong 5 \times 10^{-5}$. The hibonite samples that showed essentially no ${ }^{26} \mathrm{Al}$ also showed no ${ }^{41} \mathrm{~K}^{*}$ (see Fig. 10). These important experiments showed that ${ }^{41} \mathrm{Ca}$ and ${ }^{26} \mathrm{Al}$ were present at the same time, and also absent at the same time. Generalizing from these observations, it is necessary that both ${ }^{26} \mathrm{Al}$ and ${ }^{41} \mathrm{Ca}$ had to be present at fixed abundance levels at some early time. Therefore, the mechanisms responsible for these two nuclei appear to be coupled.

The very short lifetime of ${ }^{41} \mathrm{Ca} \quad\left(\bar{\tau}_{41} \mathrm{Ca}=0.15 \mathrm{Myr}\right)$ makes it particularly important. It can be produced both in AGB stars and by proton irradiation. Thus it might be diagnostic of an irradiation model if the $\Delta_{1}$ inferred from an AGB source were so short as to be dynamically unreasonable. However, ${ }^{41} \mathrm{Ca}$ is abundantly produced in AGB stars, with ${ }^{41} \mathrm{Ca} /{ }^{40} \mathrm{Ca} \sim 2 \times 10^{-2}$ in the production zone, as can be seen in [84]. Even for a dilution of $5 \times 10^{-3}$, $\Delta_{1}$ seems not to be forced to implausibly low values by the abundance level of ${ }^{41} \mathrm{Ca}$ in the ESS, as long as the deduced $\Delta_{1}=0.5$ to $0.8 \mathrm{Myr}$ suffices for injection and collapse.

\section{$11.5{ }^{36} \mathrm{Cl}$}

The nuclide ${ }^{36} \mathrm{Cl}\left(\bar{\tau}_{36} \mathrm{Cl}=0.43 \mathrm{Myr}\right)$ has a branched decay $\left(\beta^{-}\right.$to ${ }^{36} \mathrm{Ar}$ and $\beta^{+}$to ${ }^{36} \mathrm{~S}$ ) with the e.c. $/ \beta^{+}$branch having only a $1.9 \%$ yield. Efforts to find evidence for this nuclide were made by several groups. A study by Göbel, Begemann and Ott [247] of ${ }^{36} \mathrm{Ar}$ abundances in Allende samples (including CAIs) was done along with $\mathrm{Cl}$ measurements. These workers found large shifts in ${ }^{36} \mathrm{Ar} /{ }^{38} \mathrm{Ar}$ (up to 90 at intermediate-temperature releases). These results correlated with $\mathrm{Cl}$ and ${ }^{60} \mathrm{Co}$. If interpreted as due to ${ }^{36} \mathrm{Cl}$ decay, their results would give ${ }^{36} \mathrm{Cl} /{ }^{35} \mathrm{Cl} \sim 2 \times 10^{-8}$ (U. Ott, personal communication). A report 
by Villa et al. [248] found a sodalite-rich fine-grained inclusion called "the Pink Angel" that gave ${ }^{36} \mathrm{Cl} /{ }^{35} \mathrm{Cl}=(0.2-1.2) \times 10^{-8}$. This is the same material that gave ${ }^{129} \mathrm{I} /{ }^{127} \mathrm{I} \approx 1.0 \times 10^{-4}$. A more recent effort [249] also found effects in ${ }^{36} \mathrm{Ar}$ that were attributed to ${ }^{36} \mathrm{Cl}$. However, no strong case could be made for ${ }^{36} \mathrm{Cl}$ from this report. Interpretation of these data is unclear [250]. Recent reports by Lin et al. [251][252] demonstrated an impressive correlation of ${ }^{36} \mathrm{~S} /{ }^{34} \mathrm{~S}$ with ${ }^{35} \mathrm{Cl} /{ }^{34} \mathrm{~S}$ in late-formed halogen-rich phases in CAIs and showed it to be decoupled from ${ }^{26} \mathrm{Al}$. The results appear to demonstrate the presence of ${ }^{36} \mathrm{Cl}$ in the ESS with the very high values ${ }^{36} \mathrm{Cl} /{ }^{35} \mathrm{Cl} \cong 5 \times 10^{-6}$. If $\Delta_{1}+\Delta_{2}=(0.5-1) \mathrm{Myr}$, then the required amount is outside the range possible for an AGB source or an SN II source. We note that the problem of isobaric interferences of $\mathrm{HCl}$ with ${ }^{36} \mathrm{Cl}$ requires attention. This is a well known and persistent interference and might occur during sputtering. This would of course give a very good correlation of $\mathrm{H}^{35} \mathrm{Cl}$ with ${ }^{35} \mathrm{Cl}$. The mass resolution used by Lin et al. [252] should resolve this isobaric interference. However, because of the importance of this result, such experimental matters should be specifically addressed. The obvious conflict between the earlier reports and that of [252] is not easily reconcilable. The results by [251] and [252] are, if correct, of considerable importance and should be verified by further experiments on halogen-rich alteration phases in other CAIs and in appropriate phases in chondrites. Measurements of halite as done by Whitby et al. [136] for ${ }^{129}$ I should be carried out. There is difficulty in assigning a "time" $\Delta_{2}$ to these alteration phases. It may be possible to make determinations on phosphates or other minerals rich in halogens or other minerals where independent "age" assignments can be made from the available data. Very low or no ${ }^{26} \mathrm{Al}$ has been previously detected in sodalites, as was also found in [252], who showed that major phases with no $\mathrm{Cl}$ show the canonical value of ${ }^{26} \mathrm{Al}$ in the same CAI. From the evidence available, it appears that ${ }^{36} \mathrm{Cl}$, like ${ }^{10} \mathrm{Be}$, is decoupled from the production of ${ }^{26} \mathrm{Al}$. There is clear evidence of ${ }^{129} \mathrm{I} /{ }^{127} \mathrm{I}=1.0 \times 10^{-4}$ in CAI sodalite, but the ${ }^{129} \mathrm{I}$ life time is far too long to be useful. Correlation of ${ }^{36} \mathrm{Cl}$ with other short-lived species is much needed.

Inspection of Tables 3 and 4 shows that in AGB stars $\left({ }^{36} \mathrm{Cl} /{ }^{35} \mathrm{Cl}\right)_{E N V}$ is not dependent on the presence or absence of a ${ }^{13} \mathrm{C}$ pocket or on $Z$ for all stellar masses and $Z$ ranges studied here. Inspection of Tables 5 and 6 shows that $\left({ }^{36} \mathrm{Cl} /{ }^{35} \mathrm{Cl}\right)_{\Delta_{1}=0}=(1.7-5.4) \times 10^{-6}$ for all cases. However, due to the short lifetime, it drops to $(4.7-9.4) \times 10^{-7}$ for all LMS cases if $\Delta_{1} \sim 0.7 \mathrm{Myr}$. Thus it is not evident that an AGB source could provide the inventory that appears to be required by the data reported by Lin et al. [251] [252]. If $\Delta_{1}+\Delta_{2}=(1-2)$ Myr, then the values obtained here and in our earlier calculations [97] [84] would be low by factors from 10 to 100 . Attribution of the source of ${ }^{36} \mathrm{Cl}$ to $\mathrm{SNe}$ is not appropriate, considering the arguments given in Section 9 - the ${ }^{36} \mathrm{Cl}$ case is much like that of ${ }^{26} \mathrm{Al}$ for a $\mathrm{SN}$ source.

The basic issue is where the volatile halogen-rich alteration, common in CAIs, 
took place. It is not at all evident that this is the result of nebular processes. It might be the result of metamorphism and transport of volatile-rich materials from a heated interior penetrating the near surface layers of a protoplanet heated by ${ }^{26} \mathrm{Al}$ and ${ }^{60} \mathrm{Fe}$.

There is a further complexity related to the production of $\mathrm{Cl}$ isotopes from the abundant ${ }^{32} \mathrm{~S}$ seeds. This involves branching points at ${ }^{33} \mathrm{~S},{ }^{35} \mathrm{~S},{ }^{35} \mathrm{Cl},{ }^{36} \mathrm{Cl}$, and ${ }^{37} \mathrm{Cl}$. These involve $(\mathrm{n}, \gamma)(\mathrm{n}, \mathrm{p})$, and $(\mathrm{n}, \alpha)$ captures competing with $\beta^{ \pm}$ decays and electron captures. This is a complex issue as it depends on the decomposition of $s$-processes into main and weak components, and the general modeling of the total $s$ inventory for these nuclei. The calculations used here were based on the cross section measurements for ${ }^{34} \mathrm{~S}$ by Reifarth et al. [253] (see full discussion in that work) and the revised solar abundances [254]. For more details on the nuclear parameters and abundances of the isotopes involved in this region of the $s$-path, see Mauersberger et al. [255]. It remains to be seen whether the production of ${ }^{36} \mathrm{Cl}$ in AGB stars could conceivably be greatly increased by possible revisions to the model.

The level of ${ }^{36} \mathrm{Cl}$ production by irradiation of dust has been presented by Marhas \& Goswami [256] and Leya et al. [257]. An extensive analysis of an irradiation model that does not consider ${ }^{36} \mathrm{Cl}$ is given in [258]. If the Lin et al. [251] [252] results are confirmed, then the theoretical treatment of an irradiation model must be reconsidered as the level of ${ }^{36} \mathrm{Cl}$ production may have to be higher in comparison with other short-lived nuclei. In one report [257] the fluence required to provide the ${ }^{10} \mathrm{Be}$ appears to be commensurate with that needed to produce the ${ }^{36} \mathrm{Cl}$ abundances of Lin et al. [251] [252]. This gives ${ }^{36} \mathrm{Cl} /{ }^{35} \mathrm{Cl}=1.3 \times 10^{-4}$ for the case without saturation, but there is also a considerable shortfall in ${ }^{26} \mathrm{Al} /{ }^{27} \mathrm{Al}$, which is in accord with the absence of ${ }^{26} \mathrm{Al}$ in the sodalite with abundant ${ }^{36} \mathrm{Cl}$. In short, there is a strong need for more complete and definitive measurements of the ${ }^{36} \mathrm{Cl}$ abundance to guide future work and more efforts at modelling the production of these short-lived nuclei by particle bombardment. Calculations directed to ${ }^{36} \mathrm{Cl},{ }^{10} \mathrm{Be}$, and ${ }^{26} \mathrm{Al}$ production at the level ${ }^{36} \mathrm{Cl} /{ }^{35} \mathrm{Cl} \sim 10^{-5}$ would be useful. Careful consideration as to the conditions under which volatile-rich alteration processes might take place is urgently needed.

Note added in proof: The presence of ${ }^{36} \mathrm{Cl}$ in the Pink Angel (see Section 11.5) at the level of ${ }^{36} \mathrm{Cl} /{ }^{35} \mathrm{Cl}=(4 \pm 1) \times 10^{-6}$ has been established by W. Hsu et al. (personal communication). This sample has ${ }^{26} \mathrm{Al} /{ }^{27} \mathrm{Al}<2 \times 10^{-6}$. The ${ }^{36} \mathrm{Cl}$ is not correlated with the ${ }^{26} \mathrm{Al}$. This confirms the results by Lin et al. [252]. These results show the necessity of energetic particle bombardment in the ESS as shown by the ${ }^{10} \mathrm{Be}$ results [50]. Further, no ${ }^{36} \mathrm{Ar}$ was present in the Pink Angel, so almost complete loss of ${ }^{36} \mathrm{Ar}$ is required. 


\section{6 ${ }^{10}$ Be and Irradiation}

The nuclide ${ }^{10} \mathrm{Be} \quad \beta^{-}$decays to ${ }^{10} \mathrm{~B}\left(\bar{\tau}_{10} \mathrm{Be}=2.3 \times 10^{6} \mathrm{yr}\right)$ and is not the product of stellar nucleosynthesis. It is produced by energetic $\mathrm{H}$ and He nuclei by spallation reactions on a variety of targets (mostly $\mathrm{O}$ ). The discovery of ${ }^{10} \mathrm{Be}$ in ESS materials by McKeegan, Chaussidon \& Robert [50] in meteorites demonstrates the significance of early energetic particle bombardment by cosmic rays in providing short-lived nuclei. These cosmic rays may be from the T-Tauri phase of the proto-sun, or from outside sources [259]. As shown by McKeegan et al. [50], there is a clear correlation of ${ }^{10} \mathrm{~B} /{ }^{11} \mathrm{~B}$ with ${ }^{9} \mathrm{Be} /{ }^{11} \mathrm{~B}$ in several CAIs. This clearly establishes the presence of ${ }^{10} \mathrm{Be}$ in the ESS with ${ }^{10} \mathrm{Be} /{ }^{9} \mathrm{Be}=9.5 \times 10^{-4}$ as shown in Fig. 11 . These CAIs were previously shown to have ${ }^{26} \mathrm{Al} /{ }^{27} \mathrm{Al} \approx 5 \times 10^{-5}$. The fundamental question is whether the irradiation that produced the ${ }^{10} \mathrm{Be}$ could also be responsible for other short-lived nuclei (in particular ${ }^{26} \mathrm{Al},{ }^{36} \mathrm{Cl}$, and ${ }^{41} \mathrm{Ca}$ ). We note that the total number of ${ }^{10} \mathrm{Be}$ nuclei is very small in any sample.

To establish whether there is a correlation between ${ }^{10} \mathrm{Be}$ and ${ }^{26} \mathrm{Al}$ or ${ }^{41} \mathrm{Ca}$, studies of ultra-refractory phases in CAIs with varying levels of ${ }^{26} \mathrm{Al}$ and ${ }^{41} \mathrm{Ca}$ were conducted by Marhas et al. [260] [261] [262]. In particular, Marhas \& Goswami [261] found that the well known FUN inclusion HAL and a few other CAIs had ${ }^{10} \mathrm{Be} /{ }^{9} \mathrm{Be}$ similar to what was found in other CAIs but with two to three orders of magnitude lower ${ }^{26} \mathrm{Al} /{ }^{27} \mathrm{Al}$. This demonstrated the decoupling of ${ }^{10} \mathrm{Be}$ from ${ }^{26} \mathrm{Al}$. The available data on ${ }^{10} \mathrm{Be}$ vs. ${ }^{26} \mathrm{Al}$ are shown in Fig. 12. The conclusion from that presentation is that ${ }^{10} \mathrm{Be}$ and ${ }^{26} \mathrm{Al}$ are decoupled. This decoupling from ${ }^{26} \mathrm{Al}$ is also supported by the results for ${ }^{36} \mathrm{Cl}$ [252]. No single or uniform solar cosmic ray bombardment can explain these results, so some much more complicated scenarios must be considered.

There are now data indicating that ${ }^{26} \mathrm{Al} /{ }^{27} \mathrm{Al} \sim 5 \times 10^{-5}$ in some chondrules [236]. The search for ${ }^{10} \mathrm{Be}$ should be extended to such objects. Certainly the ${ }^{60} \mathrm{Fe}$ and ${ }^{107} \mathrm{Pd}$ and possibly ${ }^{53} \mathrm{Mn}$ require extra-solar-system nucleosynthetic sources. The isotopes ${ }^{107} \mathrm{Pd},{ }^{53} \mathrm{Mn}$, and ${ }^{26} \mathrm{Al}$ are found in planetary differentiates and require a significant inventory. The demonstration of both ${ }^{26} \mathrm{Al}$ and ${ }^{53} \mathrm{Mn}$ in the same eucrites by Nyquist et al. [238] and the possible correlation with ${ }^{207} \mathrm{~Pb} /{ }^{206} \mathrm{~Pb}$ ages is most intriguing. It now appears clear that the material in differentiated planetary bodies had significant inventories of short-lived nuclei. These nuclei must have been present in the precursor dust and other material at sufficient levels so that they were still present during planetesimal melting. This melting must be attributed to radioactive heating by ${ }^{26} \mathrm{Al}$ and some ${ }^{60} \mathrm{Fe}$. These nuclei must then have been generally present in the planetforming material of the ESS. If ${ }^{26} \mathrm{Al}$ is from an irradiation source, then this heat source must be supplied to the condensed matter making planets at least at a level of ${ }^{26} \mathrm{Al} /{ }^{27} \mathrm{Al} \sim 10^{-5}$. 
Models of irradiation of solids in the ESS have been extensively discussed by several workers [164] [256] [257] [263] [264]. There has been a major effort to seek an explanation for a large number of the short-lived nuclei by proto-solar cosmic rays impinging on dust or small rocks. There has been a particular emphasis on the X-wind model of Shu et al. [164]. Gounelle et al. [265] have explored this matter using different irradiation parameters in consideration of the ${ }^{10} \mathrm{Be}$ issue and with efforts to match the observed values of other isotopes.

It has been proposed that ${ }^{10} \mathrm{Be}$ might have its origin in galactic cosmic ray bombardment, during the collapse phase [259]. This idea is very appealing; however, it does not appear to explain the lack of correlation of ${ }^{10} \mathrm{Be}$ with ${ }^{26} \mathrm{Al}$. Indeed, if all of the material were well mixed, these isotopes would still be correlated.

The fundamental issue is whether some small fraction of debris in the early solar system was irradiated in several different episodes and not responsible for the average inventory of ${ }^{26} \mathrm{Al}$ and ${ }^{53} \mathrm{Mn}$, or if the irradiation was extensive and accounts for a substantial part of what we assume is the bulk solar inventory. In any case, the irradiation episodes had to be in a series of events to give the relationships found for ${ }^{10} \mathrm{Be},{ }^{26} \mathrm{Al}$ and ${ }^{41} \mathrm{Ca}$. The irradiation conditions proposed should be directed toward explaining and addressing the observations made on meteorites, including the decoupled production of inventories of the several nuclei.

\section{$11.7 \quad{ }^{182} \mathrm{Hf}$}

We review the situation concerning ${ }^{182} \mathrm{Hf}$, which $\beta^{-}$decays to ${ }^{182} \mathrm{~W}$. A greatly improved measurement of $\bar{\tau}_{182} \mathrm{Hf}$ by Vockenhuber et al. [266] has now established $\bar{\tau}_{182 \mathrm{Hf}}=12.8 \pm 0.1 \mathrm{Myr}$. The presence of ${ }^{182} \mathrm{Hf}$ in the early solar system was first determined by Harper \& Jacobsen [34] and by Lee \& Halliday [36] from the deficiency of ${ }^{182} \mathrm{~W} /{ }^{184} \mathrm{~W}$ in iron meteorites. For some iron meteorites ${ }^{182} \mathrm{~W} /{ }^{184} \mathrm{~W}$ was found to be $4 \epsilon \mathrm{u}$ lower than the values for the earth's crust. The inferred ESS abundance was ${ }^{182} \mathrm{Hf} /{ }^{180} \mathrm{Hf} \approx 2.84 \times 10^{-4}$. More recent studies by Kleine et al. [141] and Yin et al. [140] have demonstrated from internal isochrons on two chondrites and data on bulk samples of ordinary chondrites, a eucrite, and CAIs, that the ${ }^{182} \mathrm{Hf}$ is well-correlated with $\mathrm{Hf}-\mathrm{W}$ fractionation. These workers showed that the abundance of ${ }^{182} \mathrm{Hf} /{ }^{180} \mathrm{Hf}$ $=(1.00 \pm 0.08) \times 10^{-4}$ and that the initial $\left({ }^{182} \mathrm{~W} /{ }^{183} \mathrm{~W}\right)_{E S S}$ corresponds to $\epsilon_{W}=-3.4$ relative to samples of terrestrial rocks. It was further shown by these workers that the terrestrial samples are enriched in ${ }^{182} \mathrm{~W}$ relative to the bulk solar values resulting from the chemical fractionation of $\mathrm{Hf}$ and $\mathrm{W}$ between the earth 's metallic core and the silicate mantle while ${ }^{182} \mathrm{Hf}$ was still present (see Jacobsen [267]). These important results significantly change the 
ESS inventory of ${ }^{182} \mathrm{Hf}$ shifting ${ }^{182} \mathrm{Hf} /{ }^{180} \mathrm{Hf}$ to a lower value by a factor of $\sim 2.8$ compared to the original estimate.

A well-defined study of Hf-W by Srinivasan et al. [268] on zircon crystals $\left(\mathrm{ZrSiO}_{4}\right.$, rich in $\left.\mathrm{Hf}\right)$ from eucritic meteorites showed clear large excesses of ${ }^{182} \mathrm{~W}$ that correlated with $\mathrm{Hf} / \mathrm{W}$ and gave ${ }^{182} \mathrm{Hf} /{ }^{180} \mathrm{Hf}=1.4 \times 10^{-4}$. This is in good general agreement with the higher results cited above. This study is another example where phases with enormous enrichments of parent element relative to daughter element in ancient materials permit large isotopic effects to be observed and avoid dependence on small differences. The matter of $\Delta_{2}^{182} \mathrm{Hf}$ then remains an issue.

Studies of AGB sources of ${ }^{182} \mathrm{Hf}$ showed that it was not possible to produce the observed ${ }^{182} \mathrm{Hf} /{ }^{180} \mathrm{Hf}$, by a factor of $\sim 10^{-2}$ [97]. The new results cited above do not alter this conclusion. However, they are pertinent to the question of diverse SN sources for $r$-process nuclei. In their original report, [131] showed for a model of uniform production of $r$-process nuclei that $\left({ }^{182} \mathrm{Hf} /{ }^{180} \mathrm{Hf}\right)_{\mathrm{UP}}=$ $4.8 \times 10^{-4}$. This was based on an estimate of $57 \%$ for the $r$ contribution to ${ }^{182} \mathrm{~W}$ obtained by Gallino et al. [204] from updated cross sections and AGB models. (The previous best value, estimated by Käppeler, Beer \& Wisshak [139], had been $33 \%$.) The revised measurement of $\left({ }^{182} \mathrm{Hf} /{ }^{180} \mathrm{Hf}\right)_{E S S}=1.0 \times$ $10^{-4}$ discussed above still remains in sharp contrast to that obtained for the time for free decay of ${ }^{129} \mathrm{I}$ required by $\left({ }^{129} \mathrm{I} /{ }^{127} \mathrm{I}\right)_{E S S}=1.0 \times 10^{-4}$. The time required of $\Delta_{1}^{129} \mathrm{I} \sim 70 \times 10^{6} \mathrm{yr}$ would give $\left({ }^{182} \mathrm{Hf} /{ }^{180} \mathrm{Hf}\right)_{\mathrm{UP}} \times e^{-\Delta_{1} / \bar{\tau}_{182}}=$ $2 \times 10^{-6}$. It follows that the $r$-process source of ${ }^{182} \mathrm{Hf}$ and that of ${ }^{129} \mathrm{I}$ are not the same, as argued by [131]. Further, the new value of $\left({ }^{182} \mathrm{Hf} /{ }^{180} \mathrm{Hf}\right)_{E S S}$, combined with a continuous production model, requires that the time interval between the last $r$-process contribution to solar ${ }^{182} \mathrm{Hf}$ and the formation of solids was $18 \times 10^{6}$ years. It is now important to re-evaluate the $r$ and $s$ contributions to ${ }^{182} \mathrm{~W}$. A significant shift in the value of ${ }^{182} \mathrm{~W}_{r}$ used would change the time scale for $\Delta_{1}^{182} \mathrm{Hf}$.

\section{$11.8{ }^{92} \mathrm{Nb}$}

Evidence for the $p$ process nuclide ${ }^{92} \mathrm{Nb}$ was shown by Harper [29], who found enhanced ${ }^{92} \mathrm{Zr} /{ }^{90} \mathrm{Zr}$ in rutile $\left(\mathrm{TiO}_{2}\right)$ with a high value of $\mathrm{Nb} / \mathrm{Zr}$ in an iron meteorite with silicate inclusions. Later work by Yin et al. [269] found clear excesses of ${ }^{92} \mathrm{Zr}$ in a rutile. Münker et al. [270] found a deficiency of ${ }^{92} \mathrm{Zr}$ in some CAIs enriched in $\mathrm{Zr}$ relative to $\mathrm{Nb}$ and an excess of ${ }^{92} \mathrm{Zr}$ in phases with high $\mathrm{Nb}$ relative to $\mathrm{Zr}$. There is a serious conflict regarding the initial ${ }^{92} \mathrm{Nb} /{ }^{93} \mathrm{Nb}$ ratio. The value of ${ }^{92} \mathrm{Nb} /{ }^{93} \mathrm{Nb}$ determined by [269] and [270] was two orders of magnitude higher than reported by Harper [29]. It is difficult to attribute this value to differences in $\Delta_{2}$, as $\bar{\tau}_{92} \mathrm{Nb} \approx 52 \mathrm{Myr}$, unless the 
sample analyzed by Harper [29] was formed very late $\left(\Delta_{2}>100 \mathrm{Myr}\right)$. A further conflict has appeared with the report of Schönbächler et al. [271], who determined internal isochrons on three meteorites giving results in clear support of [29]. This matter requires further study, particularly of mineral phases showing substantial $\mathrm{Nb} / \mathrm{Zr}$ fractionation. It is not evident whether the conflict is due to analytical difficulties or to element redistribution. The possible cosmo-chronologic implications of ${ }^{92} \mathrm{Nb}$ were early considered by Minster \& Allègre [272]. However, as the yield of ${ }^{92} \mathrm{Nb}$ is quite unknown in terms of any nuclear systematics, and there is no other $\mathrm{Nb} p$-only isotope, it is only possible to come to broad chronologic conclusions.

We note that ${ }^{93} \mathrm{Nb}$ is dominantly produced by $s$-processing in AGB stars, due to the decay of ${ }^{93} \mathrm{Zr}$ in the ISM after the AGB phase has ceased. The ratio for UP is:

$$
\left(\frac{{ }^{92} \mathrm{Nb}}{{ }^{93} \mathrm{Nb}}\right)_{U P}=\frac{\bar{\tau}_{92} \mathrm{Nb}}{T}\left(\frac{p^{92} \mathrm{Nb}}{p^{93} \mathrm{Nb}}\right)_{p} f_{p}^{93 \mathrm{Nb}}=5 \times 10^{-3}\left(\frac{p^{92} \mathrm{Nb}}{p^{93} \mathrm{Nb}}\right)_{p} f_{p}^{93 \mathrm{Nb}}
$$

Here $\left(p^{92} \mathrm{Nb} / p^{93} \mathrm{Nb}\right)_{p}$ is the ratio of the $p$-process production rates and $f_{p}^{93} \mathrm{Nb}$ is the fraction of ${ }^{93} \mathrm{Nb}$ from the $p$ process. From consideration of the $s$ process it is reasonable to assume $f_{p}^{93} \mathrm{Nb} \sim 0.15$ [96]. A much larger value would cause conflicts with the Zr isotope systematics. Taking $\left(p^{92} \mathrm{Nb} / p^{93} \mathrm{Nb}\right)_{p} \sim 1$, the expected ratio is $\sim 10^{-3}$, roughly matching some of the values claimed in the literature. A much larger value is not easily understood. This matter requires clarification by well-defined efforts to find internal isochrons.

\section{The Actinides}

There are some serious problems relating to models of actinide production in "the $r$-process" and the observed relative abundances of the nuclides ${ }^{232} \mathrm{Th}$, ${ }^{238} \mathrm{U},{ }^{235} \mathrm{U},{ }^{244} \mathrm{Pu}$, and ${ }^{247} \mathrm{Cm}$ in meteorites. The production rates of the actinides are not fully understood in terms of models of $r$-process nucleosynthesis (see e.g. Goriely and Arnould [273]). For an extensive review see Cowan et al. [274] and Thielemann et al. [275]. For more general considerations see [156] regarding the problems of $r$-process nucleosynthesis, including the role of neutrinos after freezeout, and fission recycling. Observations on low-metallicity stars by Hill et al. [276] show clear evidence of non-solar $r$-process yields at high U-Th abundances. From considerations of theoretical studies we note that the yields of the actinides from their neutron rich precursors depend on models of nuclei that are far off the trajectory of beta stability, and the masses and nuclear structure of these precursor nuclei are not known. The usual approach to evaluate the abundances along the neutron flow path has been to assume 
that the abundance pattern of the precursors in the actinide region is smooth and slowly varying. The approach first used in Burbidge, Burbidge, Fowler, \& Hoyle [129] for the actinides assumes that the precursors, very neutron-rich nuclei, are essentially in equal abundance along the flow path and that the number of source nuclei may be obtained by summing the number of progenitors that do not fission and may decay into the longer-lived actinides listed above.

In all calculations presented here, we have used relative yields for the actinides and trans-actinides from Seeger et al. [277], including the estimates of oddeven effects. A recent calculation of the relative abundances of the actinides and trans-actinides by Lingenfelter et al. [278] reports essentially the same values as used here by counting progenitors following [129] and [277]. The yields calculated by [278] were obtained in a parametric $r$-process study, using a detailed model of neutron flow and beta decay and assuming that this process takes place within a core collapse $\mathrm{SN}$, in a high-entropy region.

We recognize that these quantities are not well-defined in terms either of experimental observations or of pure theory. Nonetheless, the implications of these values with respect to ESS values and implications for nucleosynthesis are very large. Thus we will pursue this approach assuming that all the estimates are correct. Note that ${ }^{244} \mathrm{Pu}$ is in the chain feeding ${ }^{232} \mathrm{Th}$ and ${ }^{247} \mathrm{Cm}$ is in the chain feeding ${ }^{235} \mathrm{U}$.

Observations on phases in meteorites give $\left({ }^{244} \mathrm{Pu} /{ }^{238} \mathrm{U}\right)_{E S S} \sim 6 \times 10^{-3}$ but with some range. A very important result by Turner et al. [279] on ancient terrestrial zircon samples unequivocally demonstrates the presence of ${ }^{244} \mathrm{Pu}$ in the early earth and gives a value $\left({ }^{244} \mathrm{Pu} /{ }^{238} \mathrm{U}\right)_{E S S}=0.0066 \pm 0.0010$ at 4.56 Gyr ago. This is important in two ways: first, it confirms the values obtained from meteorites; second, it requires that ${ }^{244} \mathrm{Pu}$ be taken into account in all models of terrestrial evolution, in particular with consideration of the isotopic abundances of ${ }^{4} \mathrm{He}$ and Xe in the earth's interior [280]. The actual value of $\left({ }^{244} \mathrm{Pu} /{ }^{232} \mathrm{Th}\right)_{E S S}$ or $\left({ }^{244} \mathrm{Pu} /{ }^{238} \mathrm{U}\right)_{E S S}$ depends on the fractionation factor for $\mathrm{Pu}$ relative to $\mathrm{Th}$ and $\mathrm{U}$ in the samples studied. A comparative study of ${ }^{244} \mathrm{Pu}$ fission products, U, Th, and light rare earth elements (LREE) was done to better assess fractionation effects and to obtain a reliable estimate of $\mathrm{Pu}$ and $\mathrm{Pu} / \mathrm{Nd}$ (cf [281]). This general fractionation issue has been discussed extensively by Burnett et al. [282]. Direct experimental work on fractionation between phases by Jones \& Burnett [283] showed that Pu behaves like a LREE and $\mathrm{Pu} / \mathrm{Sm}$ can be enhanced by a factor $\sim 2$. Again, the measured value of ${ }^{244} \mathrm{Pu} /{ }^{238} \mathrm{U}$ may be shifted from the bulk solar system value by chemical fractionation, and this remains as an uncertainty in all estimates. As ${ }^{244} \mathrm{Pu}$ was present but is now extinct in the meteorite samples, all correlations of the chemistry must use a surrogate element that is now present and behaves like $\mathrm{Pu}$ (see [281]). This is not an unambiguous assignment. Respecting this uncer- 
tainty, the measured values are all about a factor of two below the UP values (see also Table 1). This could be due to: 1) errors in the assumed relative yield pattern at ${ }^{244} \mathrm{Pu}$; 2) a time delay of $\sim 10^{8}$ yr prior to solar system formation and the last production of actinides; or 3) an error in estimating the chemical fractionation of $\mathrm{Pu}$ and $\mathrm{Th}$ in the meteoritic mineral samples analyzed. The results per se suggest a very long $\Delta_{1} \sim 10^{8}$ yr for ${ }^{244} \mathrm{Pu}$.

With regard to ${ }^{247} \mathrm{Cm}$, if there are samples of mineral phases or bulk meteorites in which there is substantial fractionation of $\mathrm{Cm}$ relative to $\mathrm{U}$ by a factor $F_{\mathrm{Cm} / \mathrm{U}}$ over the bulk solar system value, then the fractional shift in ${ }^{235} \mathrm{U} /{ }^{238} \mathrm{U}$, $\delta\left({ }^{235} \mathrm{U} /{ }^{238} \mathrm{U}\right) /\left({ }^{235} \mathrm{U} /{ }^{238} \mathrm{U}\right)_{E S S}$, observed today is

$$
\frac{\delta\left({ }^{235} \mathrm{U} /{ }^{238} \mathrm{U}\right)}{\left({ }^{235} \mathrm{U} /{ }^{238} \mathrm{U}\right)_{\mathrm{ESS}}}=\left({ }^{247} \mathrm{Cm} /{ }^{235} \mathrm{U}\right)_{E S S} F_{\mathrm{Cm} / \mathrm{U}}
$$

(cf. [284]). Estimates of $F$ depend on using a surrogate element for Cm. From the upper bounds given for the variations of ${ }^{235} \mathrm{U} /{ }^{238} \mathrm{U}$ of $4 \times 10^{-3}$ [157] [158], and using a reasonable estimate of $F,\left({ }^{247} \mathrm{Cm} /{ }^{235} \mathrm{U}\right)_{E S S}<<4 \times 10^{-3}$, or more strictly $<2 \times 10^{-3}$, was obtained. The samples included meteoritic phosphates with initially abundant ${ }^{244} \mathrm{Pu}$ present and ages of 4.56 Gyr with large fractionation of $\mathrm{Pu}, \mathrm{U}, \mathrm{Th}$ and $\mathrm{REE}$. This value may be compared with $\left({ }^{247} \mathrm{Cm} /{ }^{235} \mathrm{U}\right)_{\mathrm{UP}}=8.9 \times 10^{-3}$ (see Table 1$)$ which would imply $\Delta_{1}>2 \times 10^{7}$ years. The very recent results by Stirling et al. [159] on bulk chondritic meteorites using $\mathrm{Nd}$ as the surrogate with a range of $\mathrm{U} / \mathrm{Nd}$ of a factor of 3.5 indicate $\left({ }^{247} \mathrm{Cm} /{ }^{235} \mathrm{U}\right)_{E S S} \leq 10^{-4}$. This result would require $\Delta_{1}>5 \times 10^{7} \mathrm{yr}$ and is in accordance with the ${ }^{244} \mathrm{Pu}$ results. In no way could the gross deficiency of ${ }^{247} \mathrm{Cm}$ in the ESS found by these workers be attributed to structure in the yield pattern of the actinides, even considering the range estimated by [273]. This result then forces a longer $\Delta_{1}$ for all the actinides.

More high-precision analyses of appropriate meteoritic mineral phases showing large Nd, U, Th fractionation and containing ${ }^{244} \mathrm{Pu}$ must be done to better establish the bound. The extent to which $\mathrm{Nd} / \mathrm{U}$ or $\mathrm{Th} / \mathrm{U}$ fractionation may be used as a precise estimate of $\mathrm{Cm} / \mathrm{U}$ fractionation needs some laboratory studies. This can be done directly by determining $\mathrm{Pu}, \mathrm{Cm}, \mathrm{U}, \mathrm{Th}, \mathrm{Gd}$, and $\mathrm{Nd}$ fractionation in phases crystallizing in synthetic silicate systems using ion probe techniques and should be undertaken. Such a laboratory study can be done at relatively low activity levels.

The use of ${ }^{232} \mathrm{Th}$ as the long-lived cosmo-chronometer was pioneered and explored by [129] and [130]. This application is still an issue of importance. For ${ }^{238} \mathrm{U} /{ }^{232} \mathrm{Th}$ we must first consider the solar system value. There have been numerous measurements of this ratio in various chondritic meteorites and there is a clear dispersion in the observations. The question is: Which chondrites determine the solar ratio? The most extensive data set is by [285] [286]. It 
was found that for all chondrites, ${ }^{232} \mathrm{Th} /{ }^{238} \mathrm{U}=3.9 \pm 0.8$ (today). For $\mathrm{C} 1$ and CM2 carbonaceous chondrites, the value is $3.7 \pm 0.1$. The values of ${ }^{232} \mathrm{Th} /{ }^{238} \mathrm{U}$ estimated for the bulk earth and the moon using the abundances of ${ }^{208} \mathrm{~Pb}$ and ${ }^{206} \mathrm{~Pb}$ indicate values close to 3.8 - 4.0. Because the terrestrial and lunar materials studied are magmatic differentiates representing stages of planetary evolution, it is not possible to fix more precise values. Insofar as the carbonaceous chondrites represent the "holy grail," we use this ratio in our discussion.

From Table 1 we see that $\left({ }^{238} \mathrm{U} /{ }^{232} \mathrm{Th}\right)_{E S S}=0.438$ while for UP it is 0.388 . To explain the $13 \%$ difference would require either changing $T$ to times significantly shorter than $10^{10} \mathrm{yr}$, thereby changing the other ratios of actinides, or requiring a value of $\left({ }^{232} \mathrm{Th} /{ }^{238} \mathrm{U}\right)_{\odot}=4.18$. Alternatively, the production ratios for the actinides would have to be changed. This could most simply be accomplished by decreasing $p^{232} \mathrm{Th}$ but respecting the contributions within each decay chain. Theoretical estimates of the production of actinides will not resolve this issue.

\section{Conclusions and Major Problems - Potential and Real}

In Table 7 we summarize the inferred sources of short-lived nuclei not associated with the $r$-process. We have taken into consideration the ranges of abundances that exist in estimating the ESS inventories. We will continue to assume that the ESS values used for most nuclei represent the bulk solar inventory. Exceptions will be noted. The list gives possible sources (stellar types or particle irradiation) that can produce the nuclide or that are excluded from being the source from arguments given earlier in the text.

The exclusive cases are ${ }^{10} \mathrm{Be}$, which requires irradiation, and ${ }^{60} \mathrm{Fe}$ and ${ }^{107} \mathrm{Pd}$, which each require stellar sources. The case of ${ }^{60} \mathrm{Fe}$ is particularly important in this regard. If the source is an AGB star (with $f_{0} \sim 5 \times 10^{-3}$ ) then it also requires that the ${ }^{53} \mathrm{Mn}$ be from the ISM inventory that is the product of longerterm nucleosynthesis by SNe. There is the further requirement that the last time this ISM was replenished with ${ }^{53} \mathrm{Mn}$ by a SN was less than $10^{7}$ yr prior to formation of the ESS. The total ${ }^{60} \mathrm{Fe}$ would then be a sum of what the AGB injected and what was left after ${ }^{60} \mathrm{Fe}$ decay in the ISM. This model would give a quantitative or semi-quantitative account for all of the other nuclei listed $\left({ }^{60} \mathrm{Fe},{ }^{26} \mathrm{Al},{ }^{41} \mathrm{Ca},{ }^{107} \mathrm{Pd}\right)$ that came from stellar sources and require that they be correlated in occurrence.

Certainly the high abundance of AGB debris found in meteorites is evidence of AGB components, but does not prove they were the carriers of live nuclei. A demonstration of the presence of ${ }^{205} \mathrm{~Pb}$ would strongly support the idea that this stellar source was an AGB star. 
The ${ }^{10} \mathrm{Be}$ requires that some of the material in the solar system was subject to irradiation, possibly producing ${ }^{36} \mathrm{Cl}$, but not responsible for the major inventory of ${ }^{26} \mathrm{Al}$, at least according to current irradiation models.

If a SN source provided all the ${ }^{60} \mathrm{Fe}$ and ${ }^{53} \mathrm{Mn}$, the ${ }^{26} \mathrm{Al}$ would then have to come from irradiation or from a low mass AGB star. In the case of an AGB producing ${ }^{26} \mathrm{Al}$ and ${ }^{60} \mathrm{Fe}$, these nuclei would be the heat sources required by Urey [15]. For total heat production from ${ }^{26} \mathrm{Al}$ we have $6.0 \times 10^{12} \mathrm{erg}$ per gram of $\mathrm{Al}$, if $\left({ }^{26} \mathrm{Al} /{ }^{27} \mathrm{Al}\right)_{E S S}=5 \times 10^{-5}$. The corresponding initial heat production rate for $\mathrm{Al}$ is $5.9 \times 10^{6} \mathrm{erg} / \mathrm{yr}$ per gram of $\mathrm{Al}$. For the total heat production from ${ }^{60} \mathrm{Fe}$ we have $4.7 \times 10^{10}$ erg per gram of ${ }^{56} \mathrm{Fe}$, if ${ }^{60} \mathrm{Fe} /{ }^{56} \mathrm{Fe}=10^{-6}$. The initial heating rate is $2.1 \times 10^{10} \times\left({ }^{60} \mathrm{Fe} /{ }^{56} \mathrm{Fe}\right) \mathrm{erg} / \mathrm{yr}$ per gram of ${ }^{56} \mathrm{Fe}$. The net heat from these two nuclides can be calculated for the bulk composition of choice. The typical ratio by mass is $\mathrm{Al} / \mathrm{Fe}=0.047$ in chondritic meteorites. For this $\mathrm{Al} / \mathrm{Fe}$, the ratio of the energy produced by ${ }^{26} \mathrm{Al}$ to that by ${ }^{60} \mathrm{Fe}$ is 6. It follows that both are potent heat sources, but in these proportions, ${ }^{26} \mathrm{Al}$ is predominant. The critical matter is what the inventory of ${ }^{26} \mathrm{Al}$ and ${ }^{60} \mathrm{Fe}$ is in the material that goes to make up the planets.

Another possible scenario is that the formation of the ESS was immediately triggered by a SN, which provided all the ${ }^{60} \mathrm{Fe}$ and ${ }^{53} \mathrm{Mn}$ (but not ${ }^{26} \mathrm{Al}$ ). These two nuclei are natural products of SN models, though the quantitative yield of ${ }^{53} \mathrm{Mn}$ strongly depends on the unknown "mass cut" above which material is expelled. In any case, the total matter from the $\mathrm{SN}$ in the protosolar cloud must now be quite small $\left(f_{0} \sim 10^{-6}\right.$ to $\left.10^{-7}\right)$. This would then require that all of the other short-lived nuclei come from another source. The ${ }^{107} \mathrm{Pd}$ is certainly present in planetary differentiates and has not been identified as a product of irradiation, so it rquires a stellar source. The requirement of no ${ }^{13} \mathrm{C}$ pocket that was placed on possible AGB sources in Section 10 was used to seek coverage of several short-lived nuclei including ${ }^{26} \mathrm{Al}$ and ${ }^{41} \mathrm{Ca}$. However, in this scenario, if ${ }^{26} \mathrm{Al}$ and ${ }^{41} \mathrm{Ca}$ are attributed to irradiation of dust, then the condition of no ${ }^{13} \mathrm{C}$ pocket can be removed. The ${ }^{107} \mathrm{Pd}$ inventory can then be accounted for by a star with a "normal" ${ }^{13} \mathrm{C}$ pocket. From equation 7 and the quantities in Tables 3 and 4, we find that a very small contribution $\left(f^{107} \mathrm{Pd}=4 \times 10^{-5}\right)$ from a $1.5 M_{\odot}$ star will provide the ${ }^{107} \mathrm{Pd}$ inventory with $\Delta_{1}+\Delta_{2}=8.75 \times 10^{6}$ yr. It will also provide ${ }^{205} \mathrm{~Pb}$ with essentially the same upper bound as given in Table 4 . A similar result is found for the $3 M_{\odot}$ case. As $\bar{\tau}_{107} \mathrm{Pd}=9.4 \mathrm{Myr}$, there is then no strict requirement as to when such AGB debris needs to be added to the proto-solar cloud. This scenario is then one of a SN trigger feeding ${ }^{60} \mathrm{Fe}$ and ${ }^{53} \mathrm{Mn}$ to the cloud that had been salted with a very small amount of "normal" AGB debris within some extended interval, possibly within a $15-20 \mathrm{Myr}$ window. The ${ }^{26} \mathrm{Al},{ }^{10} \mathrm{Be},{ }^{36} \mathrm{Cl}$, and other related nuclei would then all be attributed to energetic particle irradiation of the disk. This scenario would appear to be a self-consistent story, and its implications are clear. There can only be a fraction of the ${ }^{53} \mathrm{Mn}$ inventory from 
the irradiation model. The requirement of an adequate and physically plausible irradiation scenario must provide ${ }^{10} \mathrm{Be}$ at some time but, more significantly, provide ${ }^{26} \mathrm{Al}$ to the disc at an appropriate level for a heat source. The rather sharply defined canonical value of ${ }^{26} \mathrm{Al} /{ }^{27} \mathrm{Al}$ must then be explained.

There are in any case overwhelmingly strong astrophysical observations and theoretical considerations that such particle bombardments occur around an active young star. In particular, Feigelson and his colleagues have consistently presented both observational and theoretical evidence that they must occur (see recent report by [287]). There are extensive recent studies of irradiation models referred to earlier in the text. The issue is what is a plausible and self-consistent astrophysical scenario (certainly one that does not try to explain everything, including the petrochemistry). With regard to irradiation models, we feel that some effort should be made to determine what fraction of matter in the disk was irradiated and whether this represents the total material that went into making the protoplanets and planets or is a small fraction of it. Certainly a single irradiation scenario is not acceptable considering the observations on meteorites.

In the case of a SN source for both ${ }^{60} \mathrm{Fe}$ and ${ }^{53} \mathrm{Mn}$, these nuclei should represent the abundances of the total solar system including the sun. For an AGB source, and with ${ }^{53} \mathrm{Mn}$ inherited from the ISM, the abundances should also represent global values. In the case of irradiation models, the material exposed to the particle bombardment does not represent the total solar system. There are then issues concerning the extensiveness of the irradiation. The ${ }^{26} \mathrm{Al}$ and ${ }^{53} \mathrm{Mn}$ considered here as ESS values are then only for the material that went to make up protoplanetary bodies and planets; the effective average irradiation must then be sufficient to achieve these values for the amount of "dust" required. We note that a universal value of $\left({ }^{26} \mathrm{Al} /{ }^{27} \mathrm{Al}\right)_{E S S} \sim 5 \times 10^{-5}$ has serious implications for the formation and history of cometary bodies because of its intense heat source. It is also most reasonable to expect that irradiation of material occurred only for local parcels of matter or after most protoplanetary objects had formed. In the latter case, the irradiation effects do not apply to the contents in the planets and ${ }^{26} \mathrm{Al}$ would not be a significant heat source. The ${ }^{60} \mathrm{Fe}$ coming from a stellar source would have to suffice.

The relative levels of abundances of the different nuclei and their correlation or non-correlation need better definition in irradiation models. The amounts in all materials (i.e. bulk effects seen in planets) need to be more explicitly evaluated. Scenarios with sharply-defined predictions would greatly aid in future work. Some significant efforts have been made in this direction since the work of Shu et al. [263] [264]. However the physical plausibility of these models is not evident. The consideration of the size distribution of chondrules is almost certainly a consequence of surface tension and not of $\mathrm{X}$-wind dynamics and ejection. There is now no doubt (cf. $\left.{ }^{10} \mathrm{Be}\right)$ that irradiation processes are 
important, but precisely what observations can be simply explained and what is predicted is not yet clear. The role of reprocessing of materials in and on planetesimals cannot be ignored.

The ${ }^{60} \mathrm{Fe}$ requires a stellar source; if it is from $\mathrm{SNe}$, then ${ }^{53} \mathrm{Mn}$ will certainly be co-produced and the ${ }^{53} \mathrm{Mn}$ produced by irradiation is then supplemental. From the observation of chondrules [199] there is good reason to consider that ${ }^{60} \mathrm{Fe}$ was omnipresent in the ESS. Its presence in planets as first investigated by Shukolyukov \& Lugmair [42] [43] needs to be addressed again in order to firmly establish the bulk ESS ${ }^{60} \mathrm{Fe}$ inventory. The general presence of ${ }^{60} \mathrm{Fe}$ in chondrites is being actively pursued.

If we consider the results on ${ }^{244} \mathrm{Pu} /{ }^{232} \mathrm{Th}$ and ${ }^{247} \mathrm{Cm} /{ }^{235} \mathrm{U}$ at face value then several major issues appear. If in fact the value of $\left({ }^{244} \mathrm{Pu} /{ }^{232} \mathrm{Th}\right)_{E S S}=$ $1 / 2\left({ }^{244} \mathrm{Pu} /{ }^{232} \mathrm{Th}\right)_{\mathrm{UP}}$, then this requires a $\Delta^{244} \mathrm{Pu} \approx 8 \times 10^{7}$ yr (quite close to $\left.\Delta_{129}\right)$. For this case, at the time of solar system formation $\left({ }^{247} \mathrm{Cm} /{ }^{235} \mathrm{U}\right)_{E S S}=$ $\left({ }^{247} \mathrm{Cm} /{ }^{235} \mathrm{U}\right)_{\mathrm{UP}} e^{-\Delta_{1} / \bar{\tau}_{247} \mathrm{Cm}}=2.5 \times 10^{-4}$. This would be in reasonable accord with the recent bound on ${ }^{247} \mathrm{Cm} /{ }^{235} \mathrm{U}$ required by Stirling et al. [159] from their data base. It appears evident that the last $r$-process episode producing the actinides and trans-actinides took place $\sim 10^{8} \mathrm{yr}$ before the sun formed. The critical piece of data here is the bound on $\left({ }^{247} \mathrm{Cm} /{ }^{235} \mathrm{U}\right)_{E S S}$. This number requires a long delay after the last actinide-producing event, and is independent of uncertainties in the details of production.

Assuming the above to be valid, there is a further conflict with the ${ }^{182} \mathrm{Hf}$ results, which require $\Delta_{1}^{182} \mathrm{Hf} \lesssim 2 \times 10^{7} \mathrm{yr}$. This suggests that the actinide group is made in an $r$-process distinct from the intermediate heavy mass $r$-nuclei. There would then be three regimes of $r$-process production - light $r$-nuclei (Ba and below), intermediate (Ba to intermediate heavy nuclei), and heavy (intermediate heavy nuclei - Pt group? - to actinides). Scenarios involving a blend of various $r$-process components have already been advanced [156]. As established from observations on low metallicity halo stars with high heavy $r$-process enrichment, there is no evidence of any gross variations in the abundances of all the heavy $r$-nuclei relative to the solar value. This includes $U$ and Th. However, it is now recognized that some real variability of yields exists and is to be expected across this wide mass region from $\mathrm{Ba}$ to $\mathrm{U}$. This affects the validity of $\mathrm{Th} / \mathrm{Eu}$ as a chronometer (see [147][148][145][288][155][156]). These works have demonstrated that $\mathrm{Th} / \mathrm{Eu}$ is rather variable and the notion of an exact robust heavy $r$-pattern $(\mathrm{Ba}-\mathrm{Th})$ is not valid. Consideration of the ESS inventory of $r$-nuclei is not as simple as it was long supposed to be. It is clear that no significant very heavy $r$-process event occurred close to the time that the proto-solar system formed. Some intermediate mass $r$-process must have contributed ${ }^{182} \mathrm{Hf}$ and other nuclides. The apparent coincidence of the time scale inferred for ${ }^{129} \mathrm{I}$ and that for the trans-actinides suggests that this may be due to fission [156]. The last heavy $r$ contribution would then be 
from an $r$-process that produced very heavy nuclei without fission recycling so that the yields at $\mathrm{Ba}$ and below (including I) were governed by fission. This matter requires further investigation.

The most important issue now appears to be to divine a coherent and plausible astrophysical scenario that could explain the times of different additions, in particular the late (or very late) additions of the shorter-lived nuclei from an AGB source, and the availability of matter in the ISM that was replenished by SNe II debris within $\sim 10^{7}$ years before the hypothesized AGB injection. These processes must involve neighboring molecular clouds that interacted after one was dispersed from a SNe II event, and the subsequent onset of further astration in its neighbor (see Fig. 13). It is evident from the sampling of circumstellar dust grains that material from very different molecular clouds with different metallicities must have occurred. As the lifetimes of molecular clouds are far shorter than the lifetimes of low- and intermediate-mass stars, it follows that any injection from these sources must come from stars that formed long before the molecular cloud in which the solar nebula formed.

In summary, the various radioactive nuclei present in the early solar system appear to be dominated by several distinct sources. These are: 1) The production of actinides and trans-actinides up to $\approx 10^{8}$ yr before the solar nebula formed, which then provided the ISM with the overall inventory of these nuclides. Essentially none of this group of nuclei were added later; 2) the addition of ${ }^{53} \mathrm{Mn}$ and ${ }^{60} \mathrm{Fe}$ from a more recent stellar event occurring less than $10^{7} \mathrm{yr}$ prior to formation of the sun; 3) The addition or production of a host of other short-lived nuclei, some of which appear to require a stellar source $\left({ }^{107} \mathrm{Pd}\right)$ and some of which could be produced locally in the disk by intense irradiation of dust; 4) Some irradiation of material that occurred after protoplanet formation.

There exists a compact commercial device called a "multipurpose tool" that has combinations of many individual tools all folded into a single compact pocket. It is not commonly used for repairs. Whether such an all-function device is available in the astrophysical tool box to use for the formation of the solar system remains to be seen. It always seems that one also needs some special independent tool when doing a job.

The clarification of possible and realizable scenarios now requires more active study.

Acknowledgments The authors thank the editors for their indulgence in handling this tardy submission of a very long report. The efforts of Uli Ott in providing a detailed, thorough, incisive and scholarly review have greatly improved this work. Extremely useful comments and criticisms were provided by Ian Hutcheon as well as a possible but thankfully not realized SNe shock. 
We are indebted to useful comments by F. Käppeler, J. Truran, A. McKeegan, A. Davis and M. Thiemens, as well as several other colleagues. M.E. Johnson did her usual superior work in cryptography and a special effort in re-encoding on the many versions of this manuscript. M.B. and R.G. acknowledge financial support in Italy by MIUR, under contract PRIN2004-025729. G.J.W. acknowledges the support of DOE-DE-FG03-88ER13851. K.M.N. was supported by the U.S. DOE, Nuclear Physics Division, under contract No. W-31-109-ENG-38. Caltech Division Contribution 9014(1119). 
Fig. $1 .{ }^{14} \mathrm{~N} /{ }^{15} \mathrm{~N}$ versus ${ }^{12} \mathrm{C} /{ }^{13} \mathrm{C}$ of circumstellar $\mathrm{SiC}$ grains from the data compilation generously provided by S. Amari. Only data for grains of types A and B, mainstream, and $\mathrm{Y}$ are shown ( $\sim 98 \%$ of all data), because only these are attributable to AGB stars. The solar ratios are shown by the dotted lines. The histogram at the bottom shows the relative frequency of occurrence of ${ }^{12} \mathrm{C} /{ }^{13} \mathrm{C}$ in the data, on a linear scale. Note the well-defined peak labeled MS (gray squares, Mainstream Grains). We have included the $\mathrm{Y}$ grains as part of the MS grains, with ${ }^{12} \mathrm{C} /{ }^{13} \mathrm{C}>\left({ }^{12} \mathrm{C} /{ }^{13} \mathrm{C}\right) \odot$ as they are readily explained by AGB evolution for stars with $Z$ somewhat less than $Z \odot$. The rare $\mathrm{A}$ and $\mathrm{B}$ grains (circles) with low ${ }^{12} \mathrm{C} /{ }^{13} \mathrm{C}$ and an enormous range in ${ }^{14} \mathrm{~N} /{ }^{15} \mathrm{~N}$ still require explanation. Presentation of these results is in Refs. [208] and [289] and references therein. The region accessible by AGB evolution at solar metallicity with $\mathrm{C} / \mathrm{O}>1$ is enclosed by the solid curve. The accessible regions for different values of the metallicity $Z$ and of the stellar mass are as indicated. Note that with decreasing $Z$ the envelope of accessible $\mathrm{C}-\mathrm{N}$ space extends to higher ${ }^{12} \mathrm{C} /{ }^{13} \mathrm{C}$.

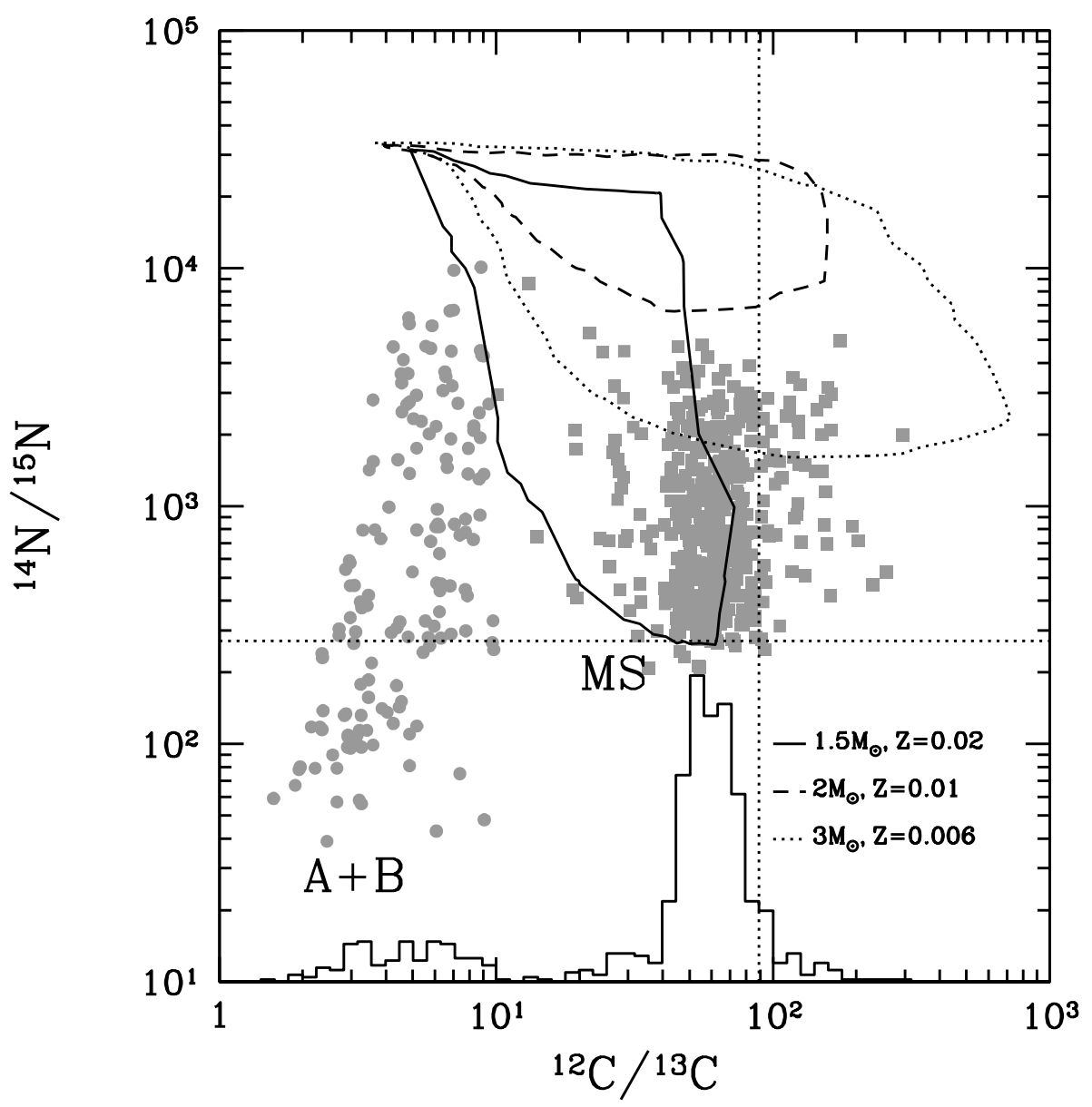


Fig. $2 .{ }^{26} \mathrm{Al} /{ }^{27} \mathrm{Al}$ versus ${ }^{12} \mathrm{C} /{ }^{13} \mathrm{C}$ for $\mathrm{SiC}$ grains as compiled by S. Amari, restricted to the same AGB-derived grain types as in Fig. 1, and again drawn from Refs. [208] and [289] and references therein. The range in ${ }^{26} \mathrm{Al} /{ }^{27} \mathrm{Al}$ is very great, from essentially no ${ }^{26} \mathrm{Al}$ to ${ }^{26} \mathrm{Al} /{ }^{27} \mathrm{Al}=1.5 \times 10^{-2}$. Balloons outlining the accessible $\mathrm{Al}-\mathrm{C}$ space are shown for three cases for $\mathrm{TDU}+\mathrm{CBP}$ with $\mathrm{C} / \mathrm{O} \geq 1$. The increase in the range of ${ }^{12} \mathrm{C} /{ }^{13} \mathrm{C}$ with decreasing $Z$ changes the boot of Italy into a loose slipper. The insert shows the range of ${ }^{26} \mathrm{Al} /{ }^{27} \mathrm{Al}$ produced by $\mathrm{CBP}$ at steady state as a function of $T_{P} / T_{H}$. We have assumed a baseline of ${ }^{26} \mathrm{Al} /{ }^{27} \mathrm{Al}=8 \times 10^{-4}$ as a minimum from normal AGB evolution without CBP (note that the total $\mathrm{H}$ shell production can be up to 5-6 times higher than this value, as discussed in the text). The cutoff in ${ }^{26} \mathrm{Al} /{ }^{27} \mathrm{Al}$ results from assuming $\log T_{P} / T_{\mathrm{H}} \leq-0.1$. The $\mathrm{A}+\mathrm{B}$ grains are problematical as it is not possible to produce the high ${ }^{26} \mathrm{Al} /{ }^{27} \mathrm{Al}$ found in many $\mathrm{B}$ grains at the low $T_{P}$ when ${ }^{12} \mathrm{C} /{ }^{13} \mathrm{C} \lesssim 10$, while maintaining $\mathrm{C} / \mathrm{O} \geq 1$.

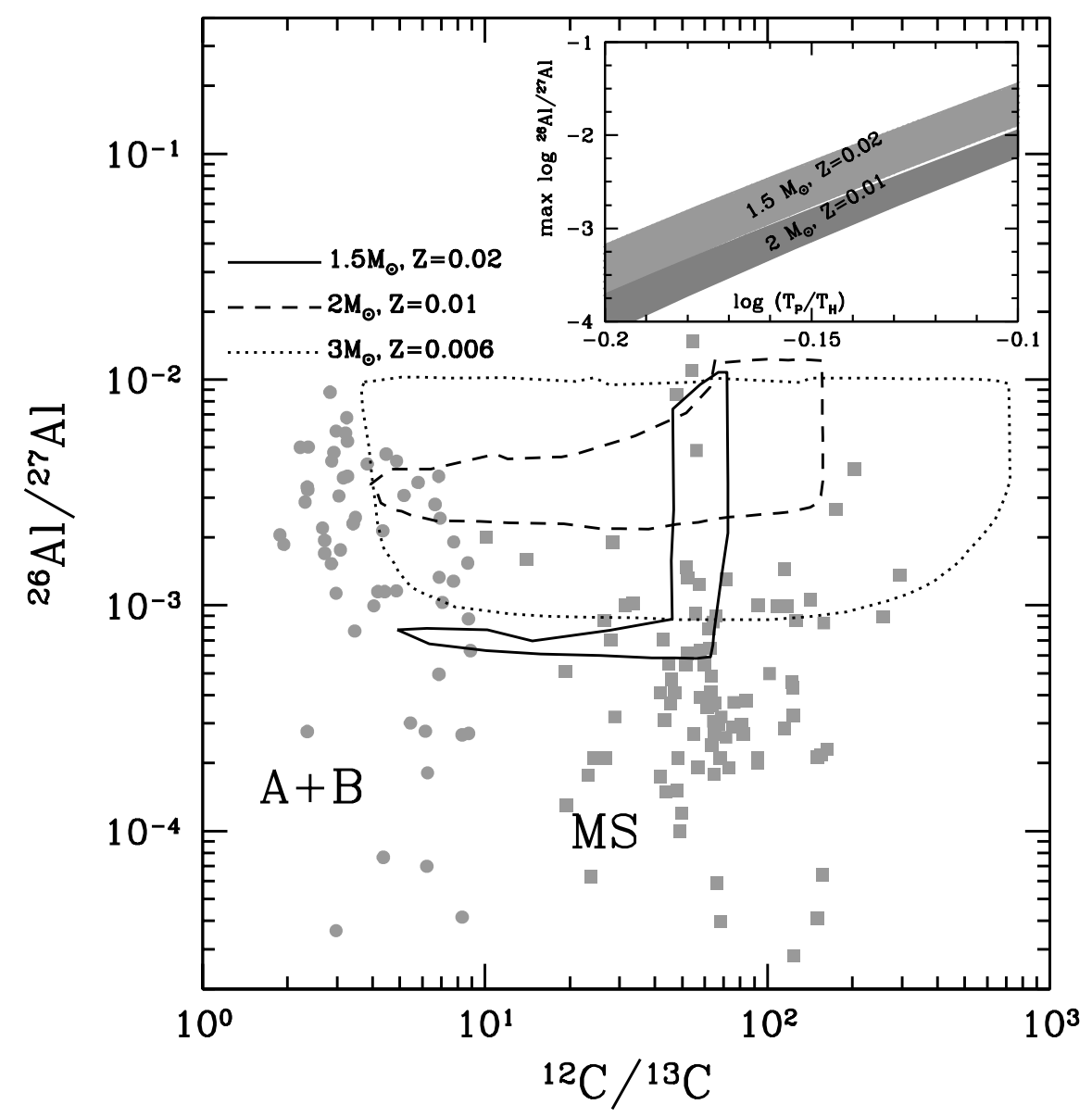


Fig. 3. The isotopic composition of $\mathrm{Mo}$ in a $\mathrm{SiC}$ grain. The values represent the per mil deviations in the isotopic composition relative to the solar values. The isotope ${ }^{96} \mathrm{Mo}$ is a pure $s$ nuclide while ${ }^{98,100} \mathrm{Mo}$ are almost exclusively $r$ process and ${ }^{92,94} \mathrm{Mo}$ are pure $p$ process nuclei. The left panel shows the measured data [62] and the right panel shows the calculated values for an $s$ process source [290]. It is evident that this carbide grain grew in the circumstellar environment of an AGB star with a very large degree of neutron exposure. (Reprinted from Nicolussi et al. [62], Copyright (1998), with permission from Elsevier.)

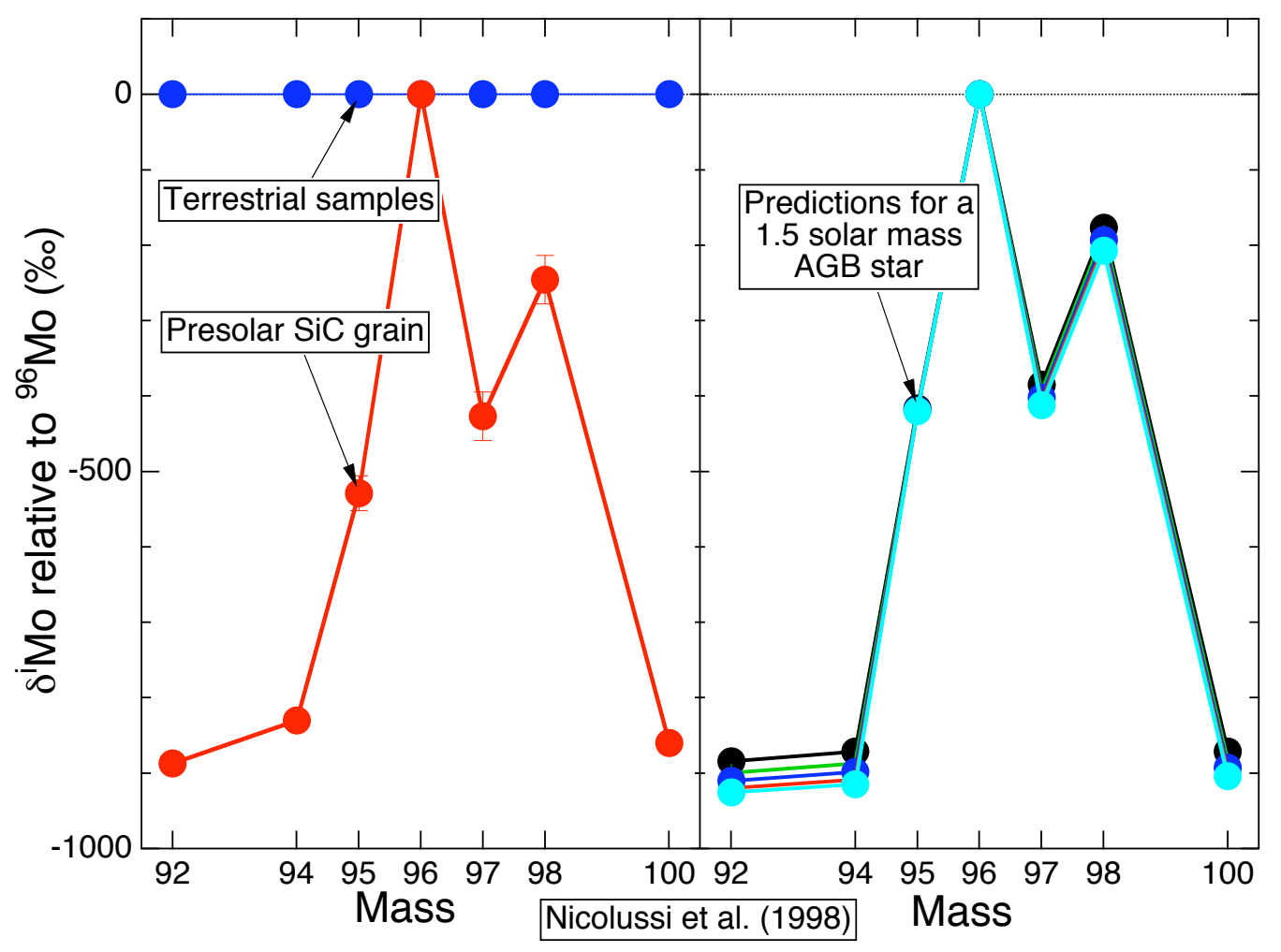


Fig. 4. ${ }^{18} \mathrm{O} /{ }^{16} \mathrm{O}$ versus ${ }^{17} \mathrm{O} /{ }^{16} \mathrm{O}$ for circumstellar oxide grains as compiled by Krestina et al. [291]. Most of the results are from Nittler et al. [71] with major contributions from Huss et al. [68], Choi et al. [82] and Krestina et al. [291]. The oxide grains represent condensates formed when $\mathrm{C} / \mathrm{O}<1$. The red dots represent data from Krestina et al. [291]. The solar ratios are as indicated. The trajectory (dashes) emanating from the solar value (labeled $Z=Z_{\odot}=0.02$ ) represents the evolution of oxygen in AGB stars with TDU but no CBP. Trajectories for different $Z$ values are also shown. The circles with black dots and associated numbers represent the stellar masses. The region labelled HBB is where hot bottom burning occurs for intermediate-mass stars. The region to the left is accessible by CBP for low-mass stars (after [102]). Note that there is a great abundance of data lying far below the trajectories accessible by normal AGB evolution, even for low $Z$. The symbols represent the phases: corundum (circles ); hibonites (diamonds); and spinel (squares). Note the absence of grains in the HBB region as ${ }^{18} \mathrm{O}$ is destroyed and ${ }^{17} \mathrm{O}$ is very greatly enhanced [102].

Figure

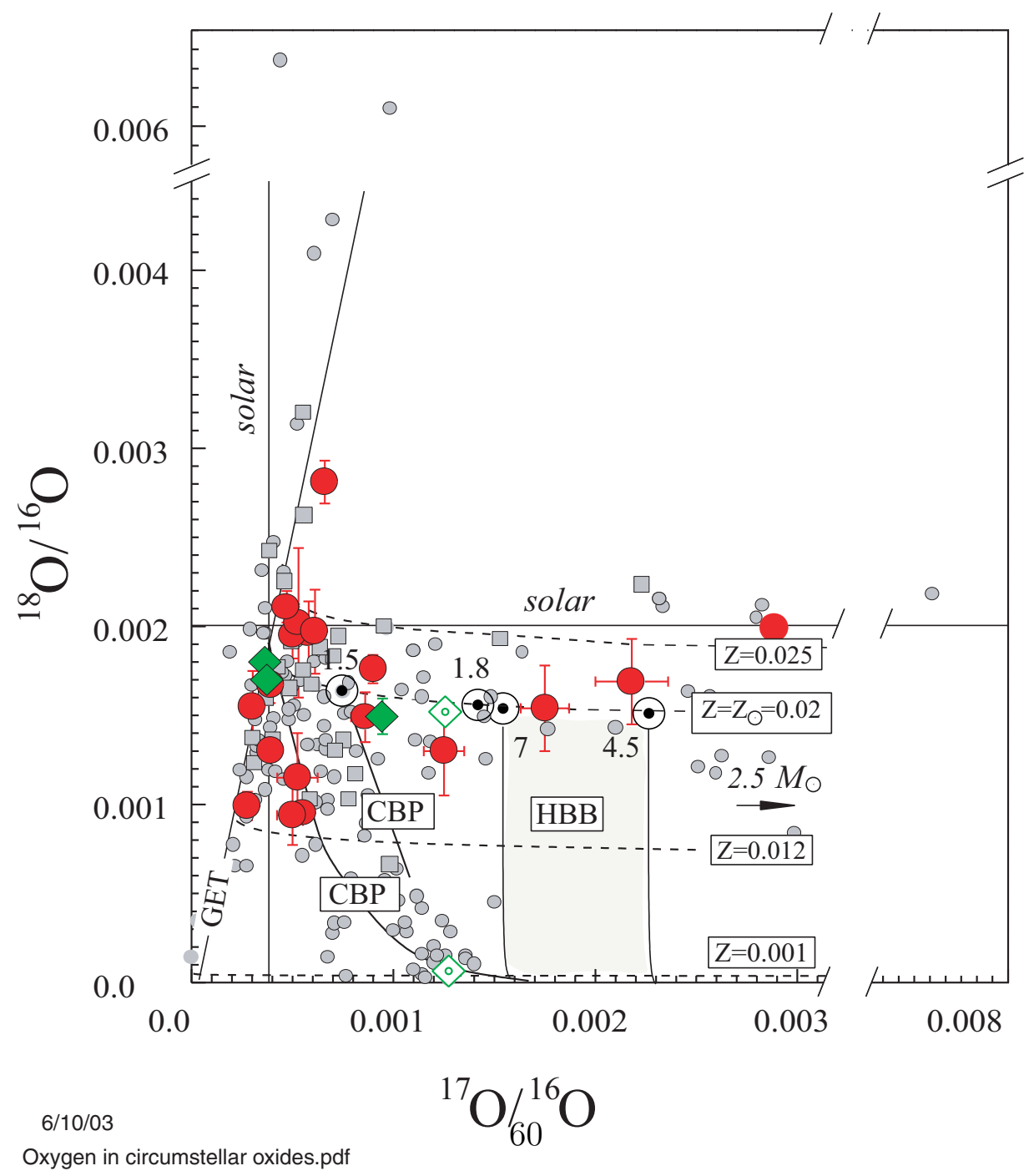


Fig. 5. Same diagram as previous figure but showing only those circumstellar oxide grains with $\mathrm{Al}$ and $\mathrm{Mg}$ isotopic data available. The value of ${ }^{26} \mathrm{Al} /{ }^{27} \mathrm{Al}$ in each grain is color indexed as indicated. The highest value observed is ${ }^{26} \mathrm{Al} /{ }^{27} \mathrm{Al}=2 \times 10^{-2}$. Note the high ${ }^{26} \mathrm{Al} /{ }^{27} \mathrm{Al}$ near the ${ }^{17} \mathrm{O} /{ }^{16} \mathrm{O}$ equilibrium value with almost no ${ }^{18} \mathrm{O}$. Also note grains (red and green) with high ${ }^{17} \mathrm{O} /{ }^{16} \mathrm{O}$ and high ${ }^{26} \mathrm{Al} /{ }^{27} \mathrm{Al}$ but with ${ }^{18} \mathrm{O}$ somewhat depleted but not destroyed. The oxygen data are clear indications of AGB evolution with $\mathrm{CBP}$ and the cases of high ${ }^{26} \mathrm{Al} /{ }^{27} \mathrm{Al}$ require $\log T_{P} / T_{\mathrm{H}} \sim-0.1$. Compilation after Krestina et al. [291].

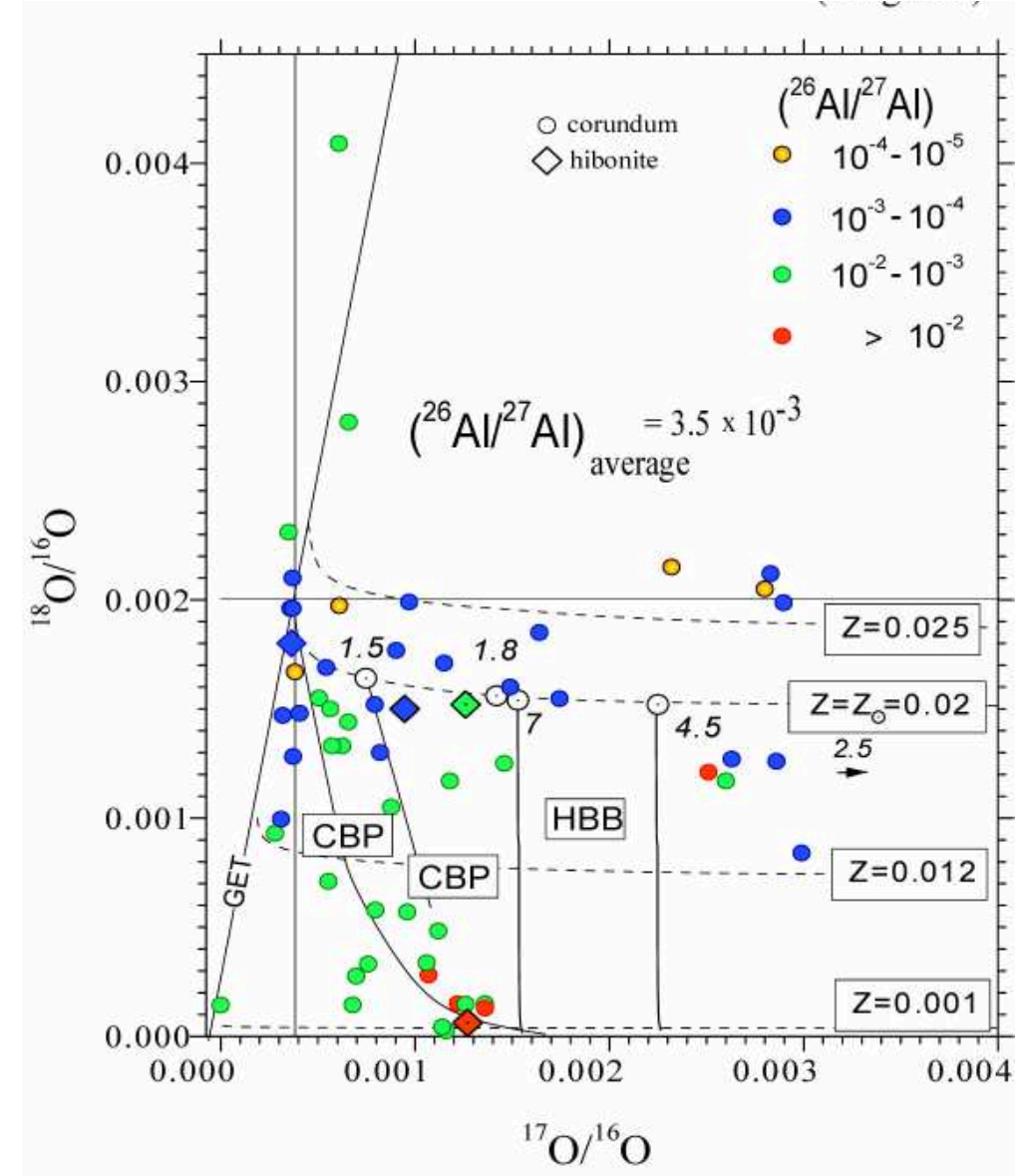


Fig. 6. The enhancement factors for stable nuclei with respect to solar concentrations for stable isotopes of interest here, as reached in the envelope of AGB star models with either low mass (upper panel) or intermediate mass (lower panel), as discussed in the text. Abundances refer to the last pulse computed. Cases are shown with a standard ${ }^{13} \mathrm{C}$ pocket (ST) and with no ${ }^{13} \mathrm{C}$ pocket.
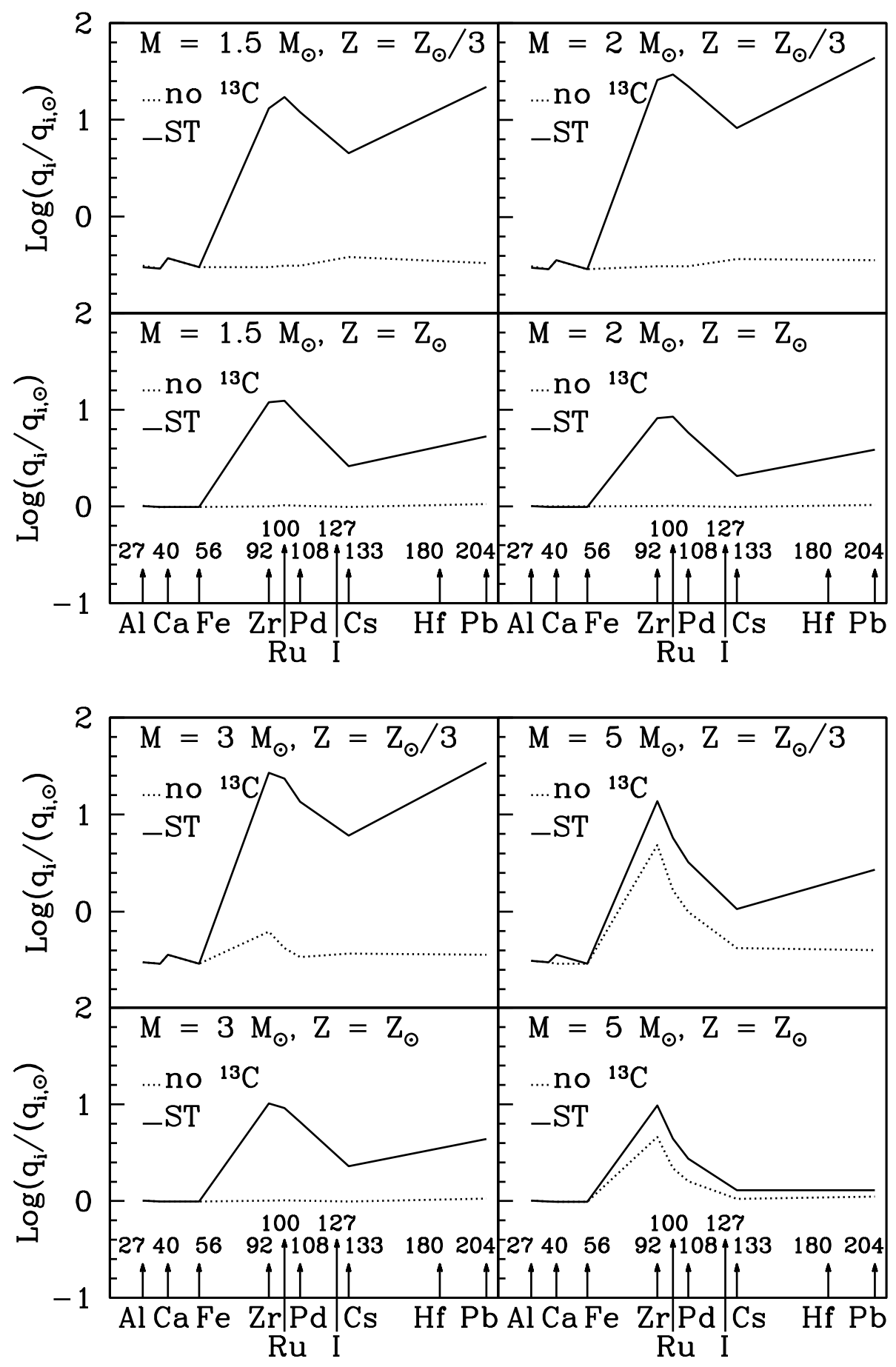
Fig. 7. The dilution factor $f_{0}$ necessary to give $\left({ }^{107} \mathrm{Pd} /{ }^{108} \mathrm{Pd}\right)_{E S S}$ with the various AGB models discussed in the text. The abscissa spans a range of initial ${ }^{107} \mathrm{Pd} /{ }^{108} \mathrm{Pd}$ ratios corresponding to $\Delta_{1}+\Delta_{2}$ between 0 and 7 Myr. Shaded areas are guides showing the typical $f_{0}$ ranges valid for LMS and IMS stars. Note the narrow range in $f_{0}$ for LMS stars.

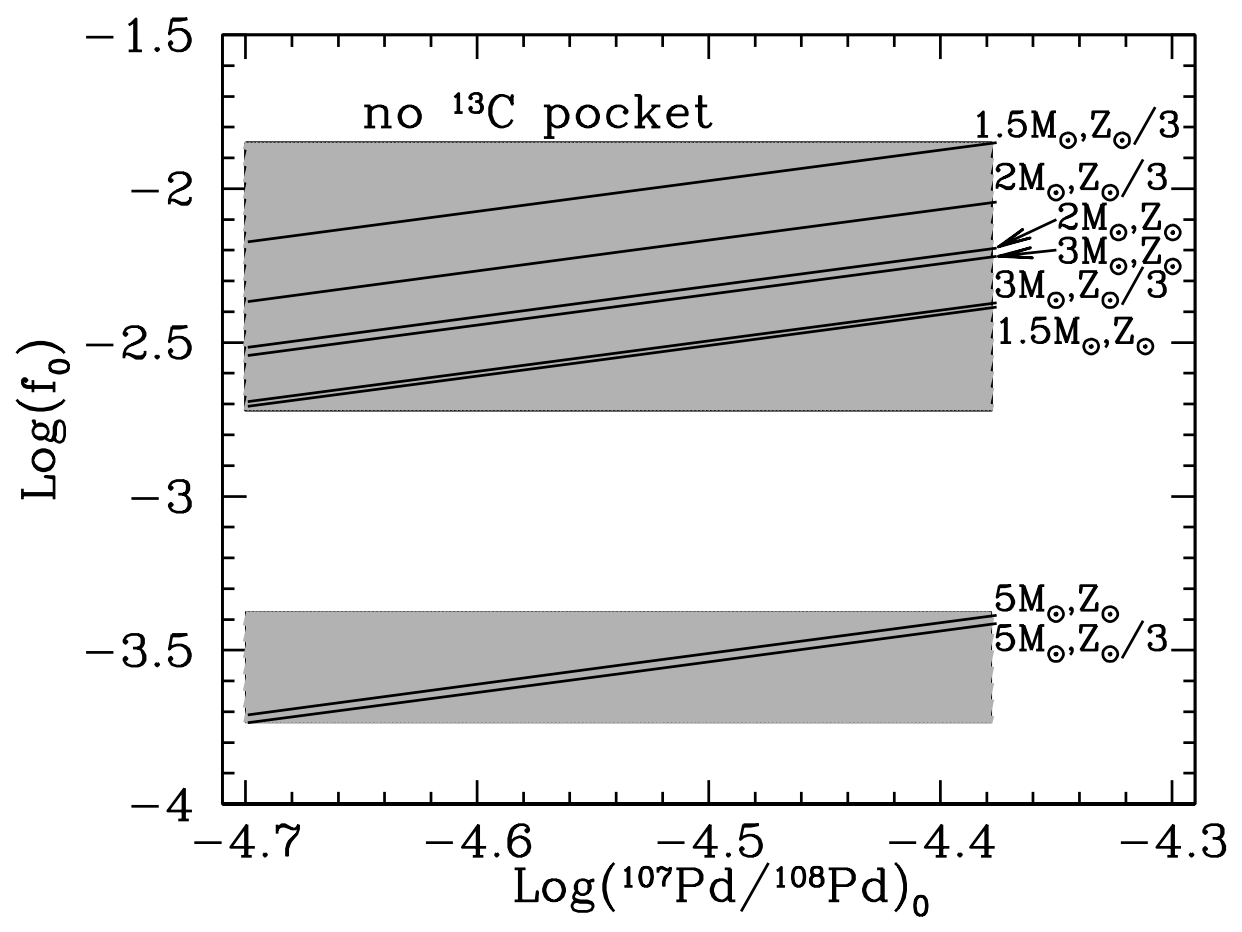


Fig. 8. The dilution factor $f_{0}$ required to produce different values of ${ }^{60} \mathrm{Fe} /{ }^{56} \mathrm{Fe}$ by addition of AGB materials to the protosolar cloud for the models discussed in the text. The abscissa spans values of initial ${ }^{60} \mathrm{Fe} /{ }^{56} \mathrm{Fe}$ in the range $(0.1-1) \times 10^{-6}$. Shaded areas are guides showing the regions covered by the dilution factor obtained for ${ }^{107} \mathrm{Pd}$ in figure 7 .

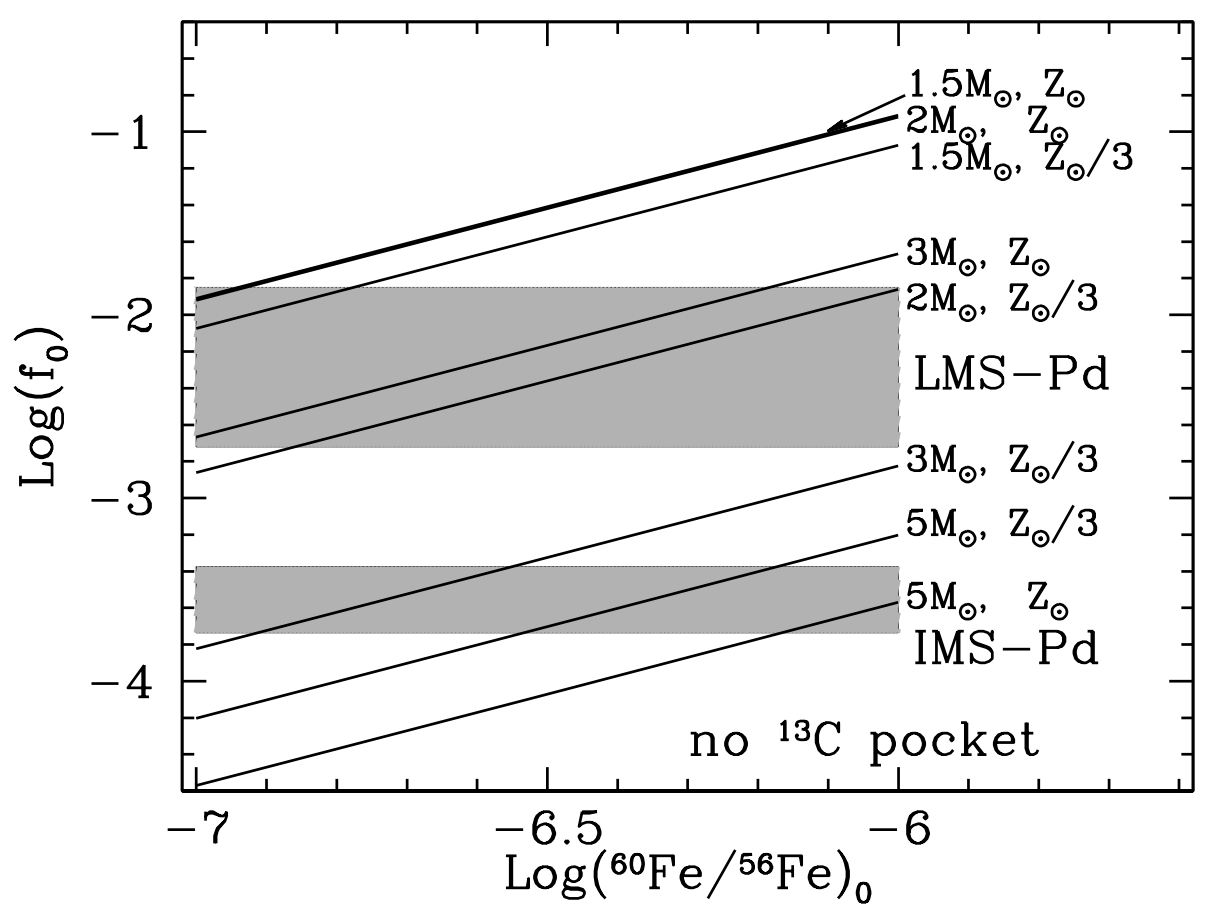


Fig. 9. a) Evolution diagram for an extinct nuclide with an initial abundance ratio $\left(N^{P} / N^{S}\right)^{0}$. P and $\mathrm{S}$ are isotopes of the same element. $\mathrm{P}$ decays to the daughter $\mathrm{D}$ of the same chemical element as I. If a system were originally isotopically homogeneous but with phases $\mathrm{A}, \mathrm{B}, \ldots \mathrm{E}$ having different ratios of the species $\mathrm{S}$ and I (representing different elements) then the existence of $\mathrm{P}$ with an abundance $\left(N^{P} / N^{S}\right)^{0}$ would require the data on $\mathrm{A}, \mathrm{B}, \ldots \mathrm{E}$ to lie on a straight line. b) The results of a CAI from Allende, showing the excellent correlation of ${ }^{26} \mathrm{Mg} /{ }^{24} \mathrm{Mg}$ with $\left({ }^{26} \mathrm{Al} /{ }^{27} \mathrm{Al}\right)_{0}=5 \times 10^{-5}$. Note small but real deviations in the inset. After [14].
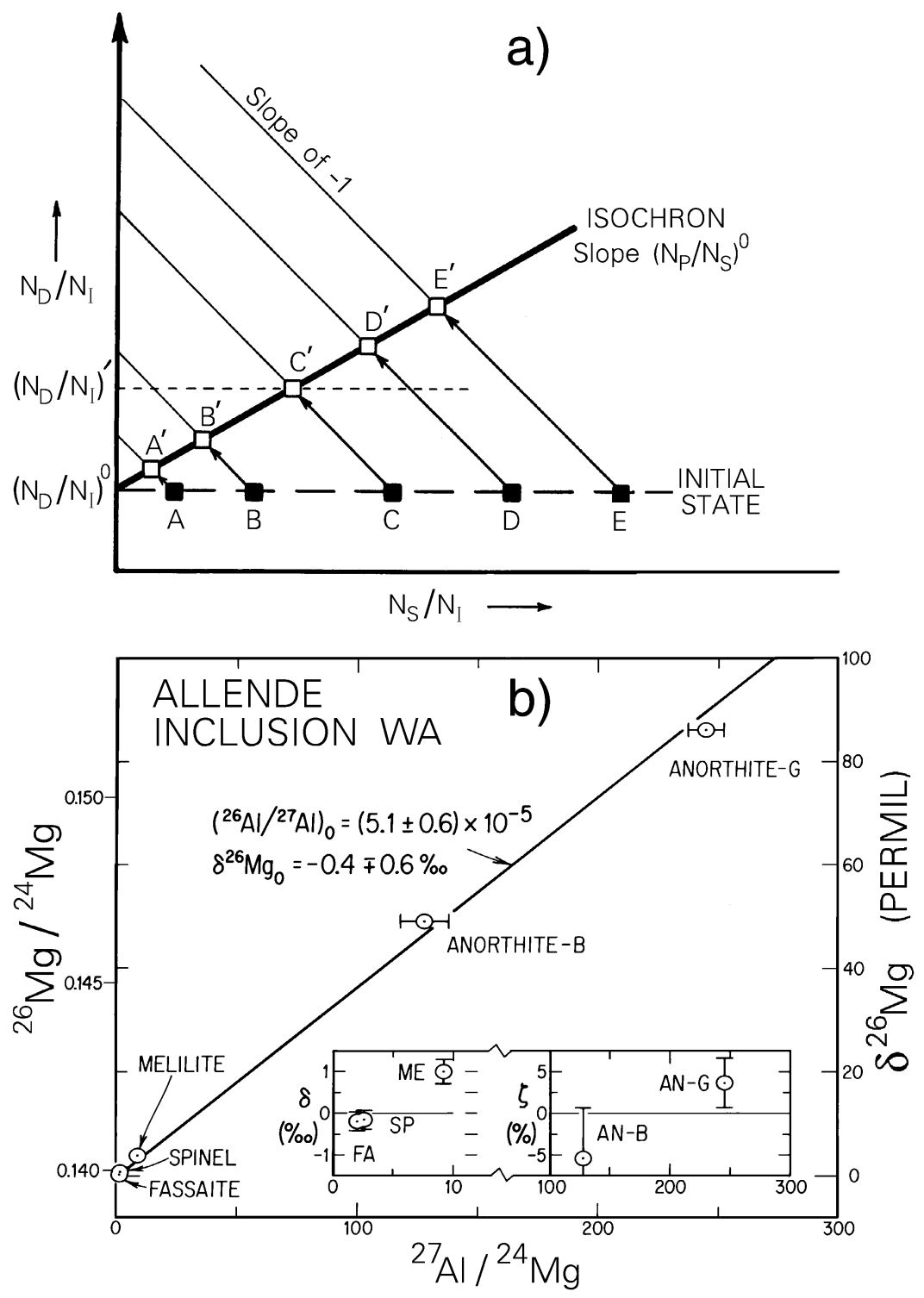
Fig. 10. Demonstration of the presence of ${ }^{41} \mathrm{Ca}$ in the ESS and its correlation with ${ }^{26} \mathrm{Al}$. Panel a) shows Al-Mg data for the CAI samples analyzed from different meteorites which exhibit the presence of ${ }^{26} \mathrm{Al}$, with ${ }^{26} \mathrm{Al} /{ }^{27} \mathrm{Al}=5 \times 10^{-5}$ (filled symbols). Note that some samples (open symbols) show no evidence of ${ }^{26} \mathrm{Al}$. Panel b) shows ${ }^{41} \mathrm{~K} /{ }^{39} \mathrm{~K}$ measurements on the same samples plotted versus ${ }^{40} \mathrm{Ca} /{ }^{39} \mathrm{~K} \times 10^{6}$. The line showing ${ }^{41} \mathrm{Ca} /{ }^{40} \mathrm{Ca}=1.4 \times 10^{-8}$ is from the original reports [48] [49]. The other data are from Sahijpal et al. [246]. These workers showed that the samples with ${ }^{26} \mathrm{Al}$ had ${ }^{41} \mathrm{Ca}$ and that those without ${ }^{26} \mathrm{Al}$ had no ${ }^{41} \mathrm{Ca}$. Note that there is limited data. The line corresponds to radioactive decay from an initial state with ${ }^{41} \mathrm{Ca} /{ }^{40} \mathrm{Ca}=1.4 \times 10^{-8}$. These data show that ${ }^{26} \mathrm{Al}$ and ${ }^{41} \mathrm{Ca}$ are well correlated and must be either co-produced or well-mixed after their production. Figure reproduced from Ref. [246], copyright Nature Publishing Group.
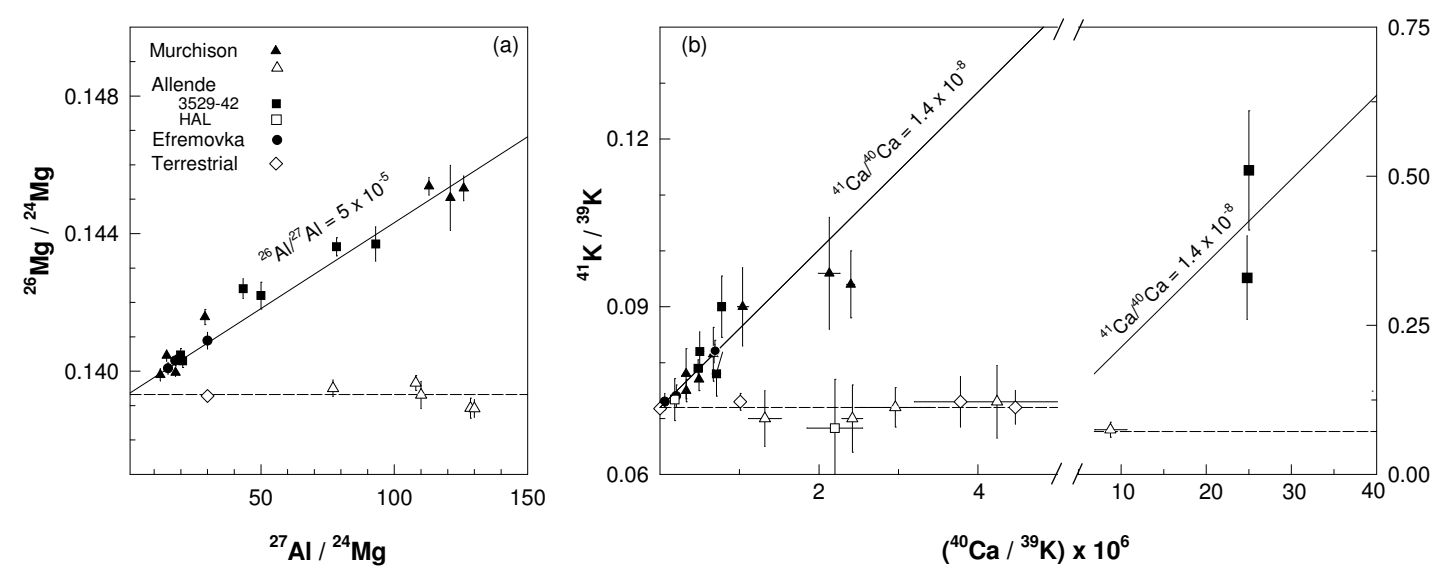
Fig. 11. Results by McKeegan, Chaussidon, and Robert [50] proving the presence of ${ }^{10} \mathrm{Be}$ in the ESS with an abundance of ${ }^{10} \mathrm{Be} /{ }^{9} \mathrm{Be} \sim 10^{-3}$. The insert shows data at low ${ }^{9} \mathrm{Be} /{ }^{11} \mathrm{~B}$ values. The ${ }^{10} \mathrm{Be}$ is only produced by particle irradiation, not by stellar nucleosynthesis. Reprinted with permission from Ref. [50]. Copyright (2000) AAAS.

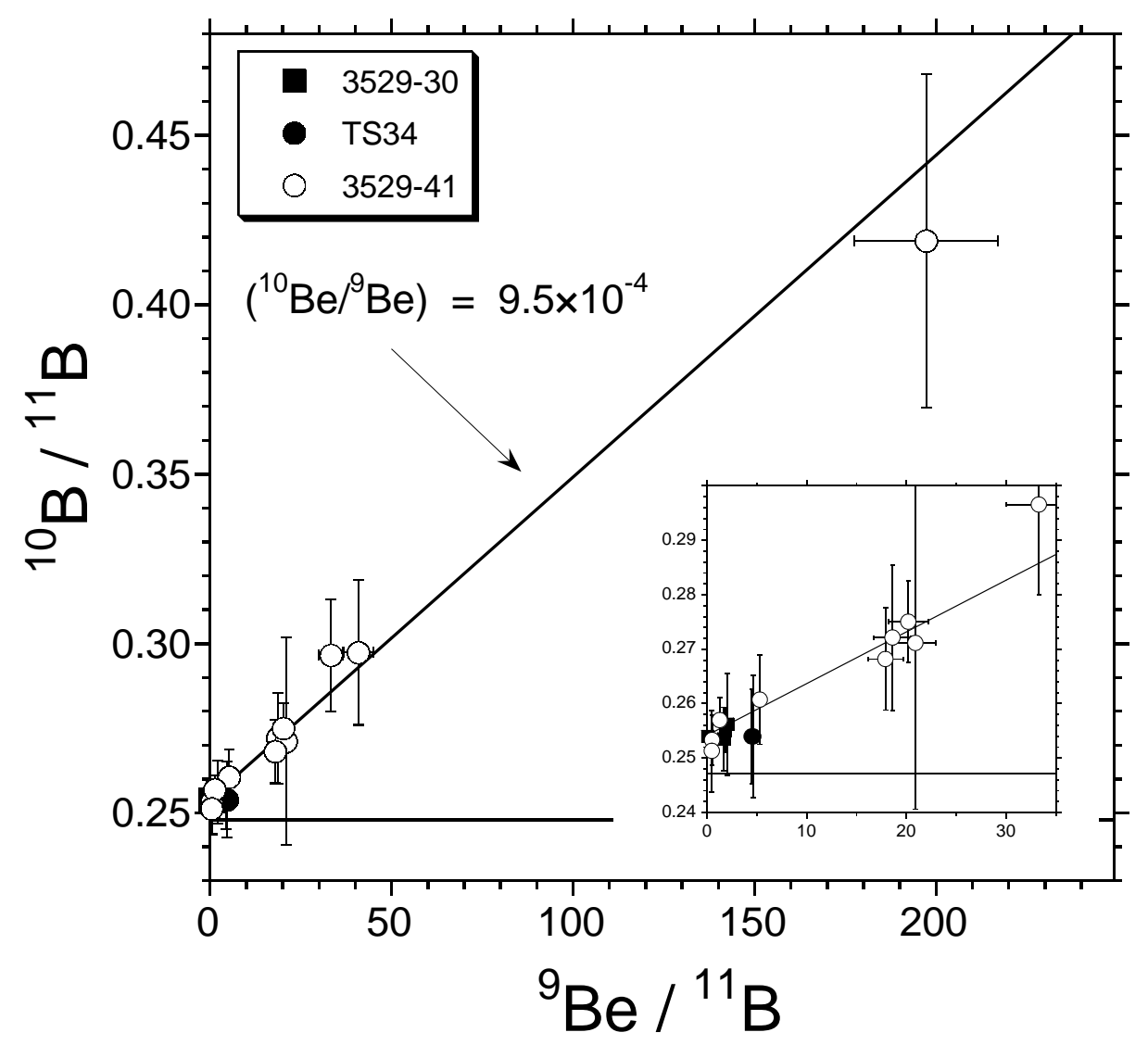


Fig. 12. Comparison of ${ }^{10} \mathrm{Be} /{ }^{9} \mathrm{Be}$ and ${ }^{26} \mathrm{Al} /{ }^{27} \mathrm{Al}$ in refractory inclusion data from several publications, compiled in Ref. [57]. These results show that samples with low or very low ${ }^{26} \mathrm{Al} /{ }^{27} \mathrm{Al}$ contain ${ }^{10} \mathrm{Be} /{ }^{9} \mathrm{Be}$ at a level compatible to that found by McKeegan et al. [50]. This shows that ${ }^{10} \mathrm{Be}$ may be present when ${ }^{26} \mathrm{Al}$ is absent. The production mechanisms or sites thereby appear unrelated. Note that there is only a limited amount of data. The full line is the time trajectory from an initial state with ${ }^{26} \mathrm{Al} /{ }^{27} \mathrm{Al}=4.5 \times 10^{-5}$ and ${ }^{10} \mathrm{Be} /{ }^{9} \mathrm{Be}=6.7 \times 10^{-4}$. (Reprinted from Ref. [57], Copyright (2003), with permission from Elsevier.)

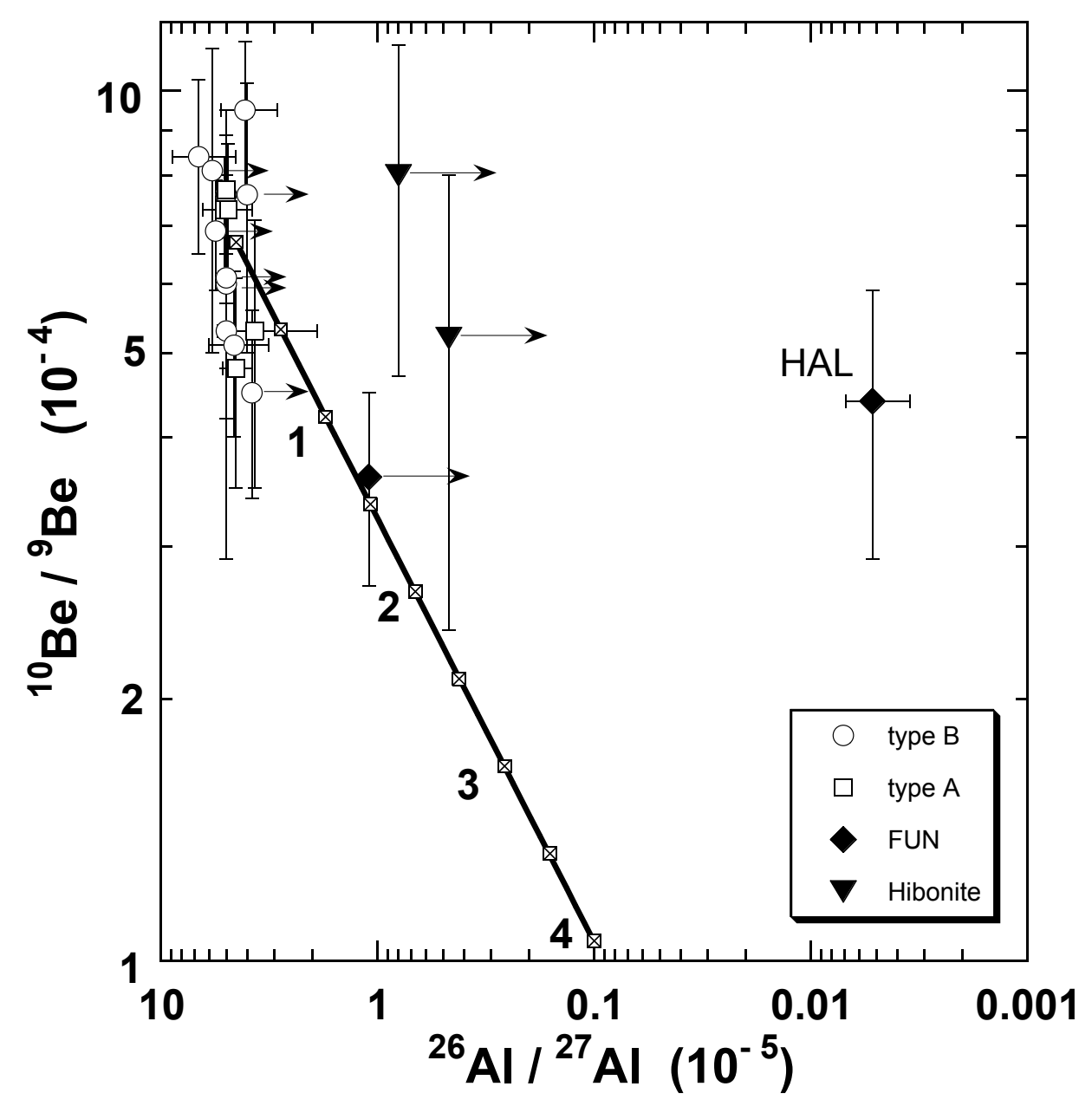


Fig. 13. Cartoon showing the complex astrophysical scenario required. There are more ancient $r$-process sites producing the actinide group and ${ }^{129} \mathrm{I}\left(\sim 10^{8}\right.$ yr before formation of the solar system); a molecular cloud that was replenished by a SN at or after $\sim 10^{7}$ yr before the solar system formed; input from an AGB star within $\sim 10^{6}$ yr before the solar system formed; energetic $p$ - and $\alpha$-capture irradiation (or several irradiations) in the early solar system. The various stellar agents that may be or may not be contributing are discussed in the text. The $r$ sources are not known, but may include low mass SNeII and accretion-induced collapse (AIC) of a binary companion to produce a neutron star.

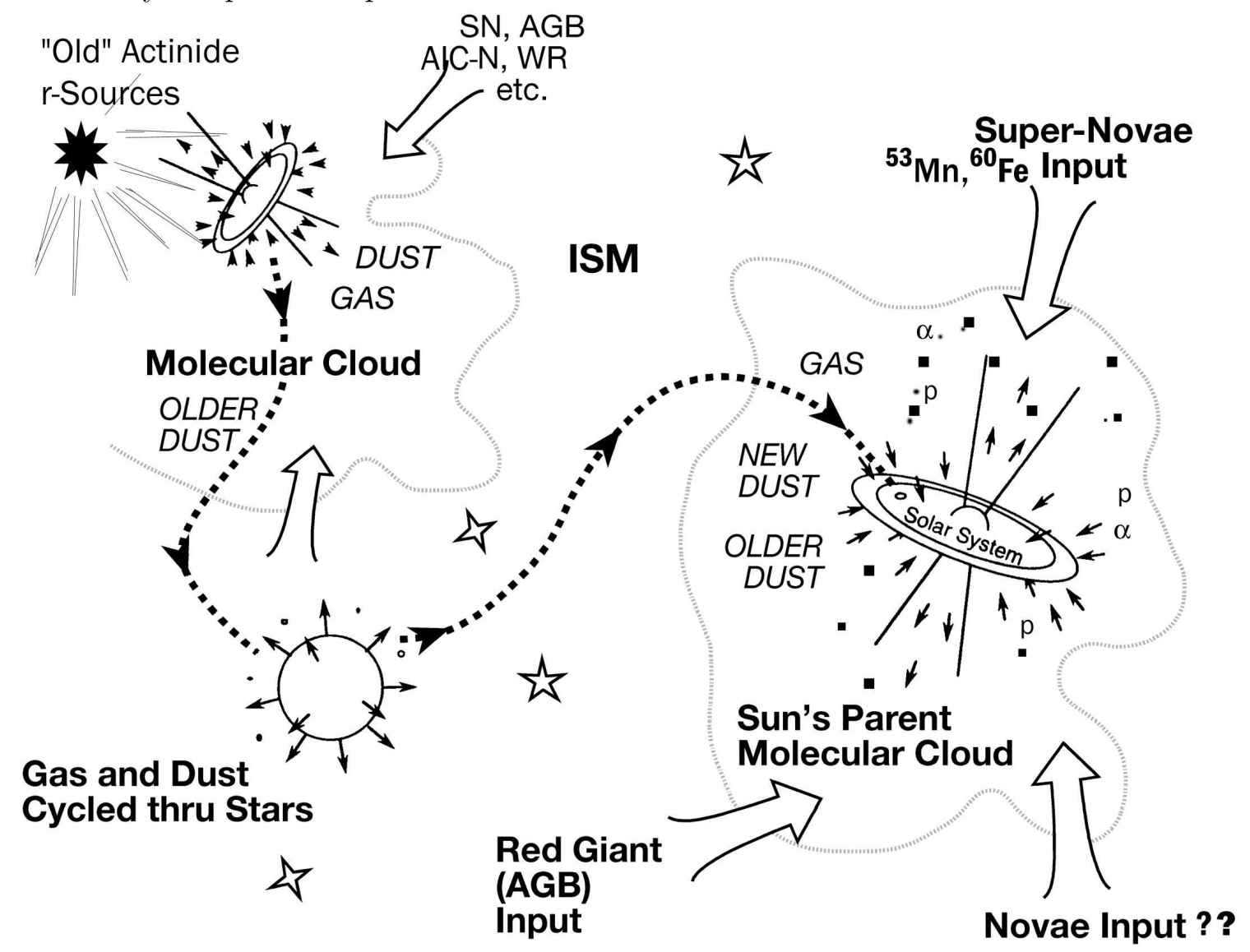


Table 1: Mean life times and abundances of short-lived nuclides, uniform production (UP) and early solar system inventory

\begin{tabular}{|c|c|c|c|c|c|c|c|c|}
\hline \multirow{2}{*}{$\begin{array}{l}\text { Radioactive } \\
\text { Isotope (R) }\end{array}$} & \multirow{2}{*}{$\begin{array}{l}\text { Reference } \\
\text { Isotope (I) }\end{array}$} & \multirow[t]{2}{*}{ Process } & \multirow{2}{*}{$\begin{array}{l}\text { Mean Life } \\
\bar{\tau}_{R}(\mathrm{Myr})\end{array}$} & \multirow[t]{2}{*}{$\left(N^{R} / N^{I}\right)_{E S S}$} & \multicolumn{4}{|c|}{$\left(N^{R} / N^{I}\right)_{U P}$} \\
\hline & & & & & $\Delta_{1}=0$ & $\Delta_{1}=5 \mathrm{Myr}$ & $\Delta_{1}=10 \mathrm{Myr}$ & $\Delta_{1}=70 \mathrm{Myr}$ \\
\hline${ }^{238} \mathrm{U}$ & ${ }^{232} \mathrm{Th}$ & $\mathrm{r} ; \mathrm{r}$ & $6.45 \times 10^{3} ; 2.03 \times 10^{4}$ & 0.438 & 0.388 & 0.388 & 0.388 & 0.388 \\
\hline${ }^{235} \mathrm{U}$ & ${ }^{238} \mathrm{U}$ & $\mathrm{r} ; \mathrm{r}$ & $1.02 \times 10^{3} ; 6.45 \times 10^{3}$ & 0.312 & 0.289 & 0.289 & 0.289 & 0.270 \\
\hline \multirow[t]{2}{*}{${ }^{244} \mathrm{Pu}$} & ${ }^{232} \mathrm{Th}$ & $r ; r$ & $115 ; 2.03 \times 10^{4}$ & $3 \times 10^{-3}$ & $5.6 \times 10^{-3}$ & $5.4 \times 10^{-3}$ & $5.1 \times 10^{-3}$ & $3.1 \times 10^{-3}$ \\
\hline & ${ }^{238} \mathrm{U}$ & $r ; r$ & $115 ; 6.45 \times 10^{3}$ & $6 \times 10^{-3}$ & $1.4 \times 10^{-2}$ & $1.3 \times 10^{-2}$ & $1.3 \times 10^{-2}$ & $7.6 \times 10^{-3}$ \\
\hline${ }^{247} \mathrm{Cm}$ & ${ }^{235} \mathrm{U}$ & $r ; r$ & $22.5 ; 1.02 \times 10^{3}$ & $\left(<2 \times 10^{-3} ;<10^{-4}\right)$ & $8.9 \times 10^{-3}$ & $7.2 \times 10^{-3}$ & $5.7 \times 10^{-3}$ & $4 \times 10^{-4}$ \\
\hline${ }^{182} \mathrm{Hf}$ & ${ }^{180} \mathrm{Hf}$ & $r ; r, s$ & $13 ;$ stable & $2.0 \times 10^{-4}$ & $4.5 \times 10^{-4}$ & $3.8 \times 10^{-4}$ & $2.1 \times 10^{-4}$ & $2 \times 10^{-6}$ \\
\hline${ }^{146} \mathrm{Sm}$ & ${ }^{144} \mathrm{Sm}$ & $p ; p$ & 148; stable & $1.0 \times 10^{-2}$ & $1.5 \times 10^{-2}$ & $1.5 \times 10^{-2}$ & $1.4 \times 10^{-2}$ & $9.4 \times 10^{-3}$ \\
\hline${ }^{92} \mathrm{Nb}$ & ${ }^{93} \mathrm{Nb}$ & $p ; s$ & $52 ;$ stable & $?$ & $1.0 \times 10^{-4}$ & $9.0 \times 10^{-5}$ & $8.2 \times 10^{-5}$ & $2.6 \times 10^{-5}$ \\
\hline${ }^{135} \mathrm{Cs}$ & ${ }^{133} \mathrm{Cs}$ & $r, s ; r, s$ & 2.9 ; stable & $1.6 \times 10^{-4} ?$ & $2.1 \times 10^{-4}$ & $3.7 \times 10^{-5}$ & $7 \times 10^{-6}$ & 0 \\
\hline${ }^{205} \mathrm{~Pb}$ & ${ }^{204} \mathrm{~Pb}$ & $\mathrm{~s} ; \mathrm{s}$ & $22 ;$ stable & $?$ & - & - & - & - \\
\hline${ }^{129} \mathrm{I}$ & ${ }^{127} \mathrm{I}$ & $r ; r, s$ & $23 ;$ stable & $1.0 \times 10^{-4}$ & $(2-5) \times 10^{-3}$ & $(1.6-4.0) \times 10^{-3}$ & $(1.4-3.5) \times 10^{-3}$ & $(1-2) \times 10^{-4}$ \\
\hline${ }^{107} \mathrm{Pd}$ & ${ }^{108} \mathrm{Pd}$ & $s, r ; r, s$ & 9.4 ; stable & $2.0 \times 10^{-5}$ & $6.2 \times 10^{-4}$ & $3.7 \times 10^{-4}$ & $2.2 \times 10^{-4}$ & $4 \times 10^{-7}$ \\
\hline${ }^{60} \mathrm{Fe}$ & ${ }^{56} \mathrm{Fe}$ & $e q, e x p, s$ & 2.2 ; stable & $\left(2 \times 10^{-7} ; 2 \times 10^{-6}\right)$ & $5 \times 10^{-7}$ & $5.2 \times 10^{-8}$ & $5.3 \times 10^{-9}$ & 0 \\
\hline${ }^{53} \mathrm{Mn}$ & ${ }^{55} \mathrm{Mn}$ & $p, \exp ; \exp$ & 5.3 ; stable & $\left(\sim 6 \times 10^{-5} ; 5 \times 10^{-6}\right)$ & $\sim 1 \times 10^{-4}$ & $4 \times 10^{-5}$ & $1.6 \times 10^{-5}$ & 0 \\
\hline${ }^{41} \mathrm{Ca}$ & ${ }^{40} \mathrm{Ca}$ & $s, \exp ; \exp$ & 0.15 ; stable & $1.5 \times 10^{-8}$ & $2 \times 10^{-8}$ & 0 & 0 & 0 \\
\hline${ }^{36} \mathrm{Cl}$ & ${ }^{35} \mathrm{Cl}$ & $s ; \exp$ & $0.43 ;$ stable & $5 \times 10^{-6}$ & $3.8 \times 10^{-7}$ & 0 & 0 & 0 \\
\hline${ }^{26} \mathrm{Al}$ & ${ }^{27} \mathrm{Al}$ & $p ; \exp$ & 1.03; stable & $5 \times 10^{-5}$ & $\sim 10^{-7}$ & 0 & 0 & 0 \\
\hline${ }^{10} \mathrm{Be}$ & ${ }^{9} \mathrm{Be}$ & spallation & $2.3 ;$ stable & $1 \times 10^{-3}$ & 0 & 0 & 0 & 0 \\
\hline
\end{tabular}


Table 2

Short-lived nuclei from a $15 M_{\odot}$ solar-metallicity SN source*

\begin{tabular}{cccccc} 
Rad. & Ref. & $q_{E N V}^{I} / q_{0}^{I}$ & $\left(N^{R} / N^{I}\right)_{E N V}$ & $\begin{array}{c}\mathrm{A} \\
\left(N^{R} / N^{I}\right)_{\Delta_{1}}\end{array}$ & $\begin{array}{c}\mathrm{B} \\
\left(N^{R} / N^{I}\right)_{\Delta_{1}}\end{array}$ \\
\hline${ }^{26} \mathrm{Al}$ & ${ }^{27} \mathrm{Al}$ & 80.7 & $5.7 \times 10^{-3}$ & $5.0 \times 10^{-5}$ & $1.2 \times 10^{-6}$ \\
${ }^{41} \mathrm{Ca}$ & ${ }^{40} \mathrm{Ca}$ & 4.7 & $1.5 \times 10^{-2}$ & $1.5 \times 10^{-8}$ & $3.5 \times 10^{-10}$ \\
${ }^{53} \mathrm{Mn}$ & ${ }^{55} \mathrm{Mn}$ & 95.6 & 0.15 & $3.5 \times 10^{-3}$ & $8 \times 10^{-5}$ \\
${ }^{107} \mathrm{Pd}$ & ${ }^{108} \mathrm{Pd}$ & 1.2 & $3.1 \times 10^{-2}$ & $1.1 \times 10^{-5}$ & $2.6 \times 10^{-7}$ \\
${ }^{60} \mathrm{Fe}$ & ${ }^{56} \mathrm{Fe}$ & 107.8 & $2.4 \times 10^{-3}$ & $4.7 \times 10^{-5}$ & $1.1 \times 10^{-6}$ \\
${ }^{36} \mathrm{Cl}$ & ${ }^{35} \mathrm{Cl}$ & 4.45 & $9.9 \times 10^{-3}$ & $1.1 \times 10^{-6}$ & $2.5 \times 10^{-8}$ \\
\hline
\end{tabular}

*Calculated from the model of Rauscher et al. [134]; ENV represents the ratio in the ejected stellar envelope.

A. Calculated to match $\left({ }^{26} \mathrm{Al} /{ }^{27} \mathrm{Al}\right)_{\Delta_{1}}=5 \times 10^{-5}$ with a dilution factor $f_{0}=$ $3.05 \times 10^{-4}, \Delta_{1}=1.09 \mathrm{Myr}$.

B. Calculated to match $\left({ }^{53} \mathrm{Mn} /{ }^{55} \mathrm{Mn}\right)_{\Delta_{1}}=1 \times 10^{-4}$ with $f_{0}=7 \times 10^{-6}, \Delta_{1}=$ 1.09 Myr. 
Table 3: $\left(q_{E N V}^{I} / q_{0}^{I}\right)$ values for a TP-AGB star

\begin{tabular}{|c|cc|cc|}
\hline \multicolumn{5}{|c|}{ Initial mass $1.5 M_{\odot}$} \\
\hline$Z / Z_{\odot}$ & \multicolumn{3}{|c|}{$1 / 3$} \\
\hline Isotope & ST & no ${ }^{13} \mathrm{C}$ pocket & ST & no ${ }^{13} \mathrm{C}$ pocket \\
\hline${ }^{27} \mathrm{Al}$ & 1.01 & 1.02 & 0.30 & 0.31 \\
${ }^{35} \mathrm{Cl}$ & 0.99 & 0.98 & 0.29 & 0.29 \\
${ }^{40} \mathrm{Ca}$ & 0.99 & 0.99 & 0.37 & 0.37 \\
${ }^{56} \mathrm{Fe}$ & 0.99 & 0.99 & 0.30 & 0.30 \\
${ }^{82} \mathrm{Kr}$ & 11.60 & 1.13 & 2.70 & 0.34 \\
${ }^{92} \mathrm{Zr}$ & 11.98 & 1.00 & 13.1 & 0.30 \\
${ }^{100} \mathrm{Ru}$ & 12.36 & 1.03 & 17.2 & 0.31 \\
${ }^{108} \mathrm{Pd}$ & 8.37 & 1.02 & 12.02 & 0.31 \\
${ }^{133} \mathrm{Cs}$ & 2.62 & 0.99 & 4.50 & 0.38 \\
${ }^{204} \mathrm{~Pb}$ & 5.30 & 1.06 & 21.90 & 0.33 \\
\hline \multicolumn{5}{|c|}{ Initial mass $2 M_{\odot}$} \\
\hline${ }^{27} \mathrm{Al}$ & 1.01 & 1.01 & 0.30 & 0.31 \\
${ }^{35} \mathrm{Cl}$ & 0.99 & 1.00 & 0.29 & 0.29 \\
${ }^{40} \mathrm{Ca}$ & 0.99 & 1.00 & 0.36 & 0.36 \\
${ }^{56} \mathrm{Fe}$ & 0.99 & 1.00 & 0.29 & 0.29 \\
${ }^{132} \mathrm{Kr}$ & 7.89 & 1.09 & 4.60 & 0.42 \\
${ }^{132} \mathrm{Cs}$ & 2.07 & 0.99 & 8.26 & 0.37 \\
${ }^{100} \mathrm{Rr}$ & 8.19 & 1.01 & 25.91 & 0.31 \\
${ }^{109} \mathrm{~Pb}$ & 3.88 & 1.04 & 43.78 & 0.36 \\
\hline
\end{tabular}




\begin{tabular}{|c|cc|cc|}
\hline \multicolumn{5}{|c|}{ Initial mass $3 M_{\odot}$} \\
\hline${ }^{27} \mathrm{Al}$ & 1.01 & 1.01 & 0.30 & 0.30 \\
${ }^{35} \mathrm{Cl}$ & 0.99 & 0.99 & 0.29 & 0.29 \\
${ }^{40} \mathrm{Ca}$ & 0.99 & 0.99 & 0.36 & 0.36 \\
${ }^{56} \mathrm{Fe}$ & 0.99 & 0.99 & 0.29 & 0.29 \\
${ }^{82} \mathrm{Kr}$ & 8.49 & 1.12 & 3.72 & 1.63 \\
${ }^{92} \mathrm{Zr}$ & 10.25 & 1.01 & 26.89 & 0.62 \\
${ }^{100} \mathrm{Ru}$ & 9.18 & 1.02 & 23.46 & 0.42 \\
${ }^{108} \mathrm{Pd}$ & 6.57 & 1.01 & 13.54 & 0.34 \\
${ }^{133} \mathrm{Cs}$ & 2.29 & 0.99 & 6.06 & 0.37 \\
${ }^{204} \mathrm{~Pb}$ & 4.37 & 1.06 & 34.32 & 0.36 \\
\hline \multicolumn{5}{|c|}{ Initial mass $5 M_{\odot}$} \\
\hline${ }^{27} \mathrm{Al}$ & 1.01 & 1.01 & 0.31 & 0.31 \\
${ }^{35} \mathrm{Cl}$ & 0.99 & 0.99 & 0.30 & 0.30 \\
${ }^{40} \mathrm{Ca}$ & 0.98 & 0.98 & 0.36 & 0.29 \\
${ }^{56} \mathrm{Fe}$ & 0.98 & 0.98 & 0.29 & 0.29 \\
${ }^{82} \mathrm{Kr}$ & 10.58 & 7.50 & 4.40 & 4.18 \\
${ }^{92} \mathrm{Zr}$ & 9.74 & 4.60 & 13.77 & 4.83 \\
${ }^{100} \mathrm{Ru}$ & 4.41 & 2.19 & 5.74 & 1.66 \\
${ }^{108} \mathrm{Pd}$ & 2.74 & 1.60 & 3.22 & 0.99 \\
${ }^{133} \mathrm{Cs}$ & 1.29 & 1.05 & 1.06 & 0.42 \\
${ }^{204} \mathrm{~Pb}$ & 1.29 & 1.11 & 2.70 & 0.40 \\
\hline
\end{tabular}


Table 4: $\left(N^{R} / N^{I}\right)_{E N V}$ values for a TP-AGB star

\begin{tabular}{|c|c|c|c|c|}
\hline \multicolumn{5}{|c|}{ Initial mass $1.5 M_{\odot}$} \\
\hline$Z / Z_{\odot}$ & \multicolumn{2}{|r|}{1} & \multicolumn{2}{|r|}{$1 / 3$} \\
\hline Isotope Pair & ST & no ${ }^{13} \mathrm{C}$ pocket & $\mathrm{ST}$ & no ${ }^{13} \mathrm{C}$ pocket \\
\hline${ }^{26} \mathrm{Al} /{ }^{27} \mathrm{Al}$ & $5.8 \times 10^{-3}$ & $5.6 \times 10^{-3}$ & $4.4 \times 10^{-3}$ & $4.3 \times 10^{-3}$ \\
\hline${ }^{36} \mathrm{Cl} /{ }^{35} \mathrm{Cl}$ & $1.4 \times 10^{-3}$ & $1.1 \times 10^{-3}$ & $7.2 \times 10^{-4}$ & $7.8 \times 10^{-4}$ \\
\hline${ }^{41} \mathrm{Ca} /{ }^{40} \mathrm{Ca}$ & $4.2 \times 10^{-4}$ & $4.5 \times 10^{-4}$ & $4.5 \times 10^{-4}$ & $4.7 \times 10^{-4}$ \\
\hline${ }^{60} \mathrm{Fe} /{ }^{56} \mathrm{Fe}$ & $7.1 \times 10^{-6}$ & $1.2 \times 10^{-5}$ & $1.0 \times 10^{-5}$ & $2.7 \times 10^{-5}$ \\
\hline${ }^{81} \mathrm{Kr} /{ }^{82} \mathrm{Kr}$ & $1.1 \times 10^{-2}$ & $3.0 \times 10^{-3}$ & $4.2 \times 10^{-3}$ & $1.3 \times 10^{-3}$ \\
\hline${ }^{93} \mathrm{Zr} /{ }^{92} \mathrm{Zr}$ & $2.6 \times 10^{-1}$ & $1.1 \times 10^{-2}$ & $2.6 \times 10^{-1}$ & $1.1 \times 10^{-2}$ \\
\hline${ }^{99} \mathrm{Tc} /{ }^{100} \mathrm{Ru}$ & $1.1 \times 10^{-1}$ & $7.0 \times 10^{-3}$ & $6.1 \times 10^{-2}$ & $3.9 \times 10^{-3}$ \\
\hline${ }^{107} \mathrm{Pd} /{ }^{108} \mathrm{Pd}$ & $1.4 \times 10^{-1}$ & $1.0 \times 10^{-2}$ & $1.5 \times 10^{-1}$ & $9.6 \times 10^{-3}$ \\
\hline${ }^{135} \mathrm{Cs} /{ }^{133} \mathrm{Cs}$ & $3.3 \times 10^{-1}$ & $1.8 \times 10^{-2}$ & $5.1 \times 10^{-1}$ & $1.6 \times 10^{-2}$ \\
\hline${ }^{205} \mathrm{~Pb} /{ }^{204} \mathrm{~Pb}$ & $1.0 \times 10^{0}$ & $1.0 \times 10^{-1}$ & $1.2 \times 10^{0}$ & $1.1 \times 10^{-1}$ \\
\hline \multicolumn{5}{|c|}{ Initial mass $2 M_{\odot}$} \\
\hline${ }^{26} \mathrm{Al} /{ }^{27} \mathrm{Al}$ & $3.6 \times 10^{-3}$ & $3.4 \times 10^{-3}$ & $5.0 \times 10^{-3}$ & $5.1 \times 10^{-3}$ \\
\hline${ }^{36} \mathrm{Cl} /{ }^{35} \mathrm{Cl}$ & $6.5 \times 10^{-4}$ & $7.3 \times 10^{-4}$ & $1.5 \times 10^{-3}$ & $1.2 \times 10^{-3}$ \\
\hline${ }^{41} \mathrm{Ca} /{ }^{40} \mathrm{Ca}$ & $2.6 \times 10^{-4}$ & $2.8 \times 10^{-4}$ & $7.6 \times 10^{-4}$ & $2.5 \times 10^{-4}$ \\
\hline${ }^{60} \mathrm{Fe} /{ }^{56} \mathrm{Fe}$ & $4.6 \times 10^{-6}$ & $8.3 \times 10^{-6}$ & $1.3 \times 10^{-4}$ & $2.5 \times 10^{-4}$ \\
\hline${ }^{81} \mathrm{Kr} /{ }^{82} \mathrm{Kr}$ & $7.9 \times 10^{-3}$ & $1.5 \times 10^{-3}$ & $2.7 \times 10^{-3}$ & $2.4 \times 10^{-3}$ \\
\hline${ }^{93} \mathrm{Zr} /{ }^{92} \mathrm{Zr}$ & $1.3 \times 10^{-1}$ & $6.5 \times 10^{-3}$ & $2.6 \times 10^{-1}$ & $2.3 \times 10^{-2}$ \\
\hline${ }^{99} \mathrm{Tc} /{ }^{100} \mathrm{Ru}$ & $7.6 \times 10^{-2}$ & $3.1 \times 10^{-3}$ & $5.0 \times 10^{-2}$ & $5.4 \times 10^{-3}$ \\
\hline${ }^{107} \mathrm{Pd} /{ }^{108} \mathrm{Pd}$ & $1.3 \times 10^{-1}$ & $6.5 \times 10^{-3}$ & $1.5 \times 10^{-1}$ & $1.5 \times 10^{-2}$ \\
\hline${ }^{135} \mathrm{Cs} /{ }^{133} \mathrm{Cs}$ & $2.7 \times 10^{-1}$ & $1.0 \times 10^{-2}$ & $6.6 \times 10^{-1}$ & $3.1 \times 10^{-2}$ \\
\hline${ }^{205} \mathrm{~Pb} /{ }^{204} \mathrm{~Pb}$ & $9.4 \times 10^{-1}$ & $6.7 \times 10^{-2}$ & $1.3 \times 10^{0}$ & $2.3 \times 10^{-1}$ \\
\hline
\end{tabular}




\begin{tabular}{|c|c|c|c|c|}
\hline \multicolumn{5}{|c|}{ Initial mass $3 M_{\odot}$} \\
\hline${ }^{26} \mathrm{Al} /{ }^{27} \mathrm{Al}$ & $2.9 \times 10^{-3}$ & $2.8 \times 10^{-3}$ & $3.5 \times 10^{-3}$ & $3.5 \times 10^{-3}$ \\
\hline${ }^{36} \mathrm{Cl} /{ }^{35} \mathrm{Cl}$ & $6.8 \times 10^{-4}$ & $7.3 \times 10^{-4}$ & $1.5 \times 10^{-3}$ & $1.5 \times 10^{-3}$ \\
\hline${ }^{41} \mathrm{Ca} /{ }^{40} \mathrm{Ca}$ & $3.0 \times 10^{-4}$ & $3.2 \times 10^{-4}$ & $4.0 \times 10^{-4}$ & $4.1 \times 10^{-4}$ \\
\hline${ }^{60} \mathrm{Fe} /{ }^{56} \mathrm{Fe}$ & $2.8 \times 10^{-5}$ & $4.7 \times 10^{-5}$ & $1.6 \times 10^{-3}$ & $2.3 \times 10^{-3}$ \\
\hline${ }^{81} \mathrm{Kr} /{ }^{82} \mathrm{Kr}$ & $4.8 \times 10^{-3}$ & $1.2 \times 10^{-3}$ & $5.7 \times 10^{-3}$ & $1.0 \times 10^{-2}$ \\
\hline${ }^{93} \mathrm{Zr} /{ }^{92} \mathrm{Zr}$ & $2.4 \times 10^{-1}$ & $9.1 \times 10^{-3}$ & $2.6 \times 10^{-1}$ & $1.3 \times 10^{-1}$ \\
\hline${ }^{99} \mathrm{Tc} /{ }^{100} \mathrm{Ru}$ & $7.6 \times 10^{-2}$ & $3.7 \times 10^{-3}$ & $1.2 \times 10^{-1}$ & $6.3 \times 10^{-2}$ \\
\hline${ }^{107} \mathrm{Pd} /{ }^{108} \mathrm{Pd}$ & $1.3 \times 10^{-1}$ & $6.9 \times 10^{-3}$ & $1.5 \times 10^{-1}$ & $2.9 \times 10^{-2}$ \\
\hline${ }^{135} \mathrm{Cs} /{ }^{133} \mathrm{Cs}$ & $4.4 \times 10^{-1}$ & $1.8 \times 10^{-2}$ & $8.7 \times 10^{-1}$ & $3.0 \times 10^{-2}$ \\
\hline${ }^{205} \mathrm{~Pb} /{ }^{204} \mathrm{~Pb}$ & $1.0 \times 10^{0}$ & $9.1 \times 10^{-2}$ & $1.1 \times 10^{0}$ & $2.4 \times 10^{-1}$ \\
\hline \multicolumn{5}{|c|}{ Initial mass $5 M_{\odot}$} \\
\hline${ }^{26} \mathrm{Al} /{ }^{27} \mathrm{Al}$ & $5.3 \times 10^{-4}$ & $5.3 \times 10^{-4}$ & $6.6 \times 10^{-4}$ & $6.3 \times 10^{-4}$ \\
\hline${ }^{36} \mathrm{Cl} /{ }^{35} \mathrm{Cl}$ & $1.1 \times 10^{-3}$ & $1.1 \times 10^{-3}$ & $1.9 \times 10^{-3}$ & $1.4 \times 10^{-3}$ \\
\hline${ }^{41} \mathrm{Ca} /{ }^{40} \mathrm{Ca}$ & $1.2 \times 10^{-4}$ & $1.2 \times 10^{-4}$ & $1.0 \times 10^{-4}$ & $7.9 \times 10^{-5}$ \\
\hline${ }^{60} \mathrm{Fe} /{ }^{56} \mathrm{Fe}$ & $3.6 \times 10^{-3}$ & $3.8 \times 10^{-3}$ & $4.8 \times 10^{-3}$ & $5.5 \times 10^{-3}$ \\
\hline${ }^{81} \mathrm{Kr} /{ }^{82} \mathrm{Kr}$ & $7.1 \times 10^{-3}$ & $7.5 \times 10^{-3}$ & $6.8 \times 10^{-3}$ & $9.4 \times 10^{-3}$ \\
\hline${ }^{93} \mathrm{Zr} /{ }^{92} \mathrm{Zr}$ & $2.3 \times 10^{-1}$ & $1.9 \times 10^{-1}$ & $2.6 \times 10^{-1}$ & $2.5 \times 10^{-1}$ \\
\hline${ }^{99} \mathrm{Tc} /{ }^{100} \mathrm{Ru}$ & $1.3 \times 10^{-1}$ & $9.7 \times 10^{-2}$ & $1.6 \times 10^{-1}$ & $1.5 \times 10^{-1}$ \\
\hline${ }^{107} \mathrm{Pd} /{ }^{108} \mathrm{Pd}$ & $1.1 \times 10^{-1}$ & $6.4 \times 10^{-2}$ & $1.5 \times 10^{-1}$ & $1.1 \times 10^{-1}$ \\
\hline${ }^{135} \mathrm{Cs} /{ }^{133} \mathrm{Cs}$ & $2.9 \times 10^{-1}$ & $8.3 \times 10^{-2}$ & $8.0 \times 10^{-2}$ & $3.0 \times 10^{-1}$ \\
\hline${ }^{205} \mathrm{~Pb} /{ }^{204} \mathrm{~Pb}$ & $3.0 \times 10^{-1}$ & $1.6 \times 10^{-1}$ & $9.1 \times 10^{-1}$ & $3.1 \times 10^{-1}$ \\
\hline
\end{tabular}


Table 5: Abundances of short-lived nuclei in a cloud salted with ejecta from an AGB star $\left(\right.$ no $\left.{ }^{13} \mathrm{C}\right)$

\begin{tabular}{|c|c|c|c|}
\hline \multicolumn{4}{|c|}{$f_{0}=5 \times 10^{-3} ;\left(1.5 M_{\odot}, Z_{\odot}\right)$} \\
\hline$\left(N^{R} / N^{I}\right)_{\Delta_{1}}$ & $\Delta_{1}=0$ & $\Delta_{1}=0.75 \mathrm{Myr}$ & $\Delta_{1}+\Delta_{2}=8.75 \mathrm{Myr}$ \\
\hline${ }^{26} \mathrm{Al} /{ }^{27} \mathrm{Al}\left(2 \times 10^{-2}\right)^{*}$ & $\left(1.0 \times 10^{-4}\right)$ & $\left(5 \times 10^{-5}\right)$ & $\left(2.5 \times 10^{-8}\right)$ \\
\hline${ }^{36} \mathrm{Cl} /{ }^{35} \mathrm{Cl}$ & $5.4 \times 10^{-6}$ & $9.4 \times 10^{-7}$ & - \\
\hline${ }^{41} \mathrm{Ca} /{ }^{40} \mathrm{Ca}$ & $2.2 \times 10^{-6}$ & $\left(1.5 \times 10^{-8}\right)^{\dagger}$ & - \\
\hline${ }^{60} \mathrm{Fe} /{ }^{56} \mathrm{Fe}$ & $5.9 \times 10^{-8}$ & $4.2 \times 10^{-8}$ & $1.1 \times 10^{-9}$ \\
\hline${ }^{81} \mathrm{Kr} /{ }^{82} \mathrm{Kr}$ & $1.7 \times 10^{-5}$ & $1.4 \times 10^{-6}$ & - \\
\hline${ }^{93} \mathrm{Zr} /{ }^{92} \mathrm{Zr}$ & $5.5 \times 10^{-5}$ & $3.9 \times 10^{-5}$ & $1.0 \times 10^{-6}$ \\
\hline${ }^{99} \mathrm{Tc} /{ }^{100} \mathrm{Ru}$ & $3.6 \times 10^{-5}$ & $2.7 \times 10^{-6}$ & - \\
\hline${ }^{107} \mathrm{Pd} /{ }^{108} \mathrm{Pd}$ & $5.1 \times 10^{-5}$ & $4.7 \times 10^{-5}$ & $\left(2.0 \times 10^{-5}\right)^{* *}$ \\
\hline${ }^{135} \mathrm{Cs} /{ }^{133} \mathrm{Cs}$ & $8.9 \times 10^{-5}$ & $6.9 \times 10^{-5}$ & $4.4 \times 10^{-6}$ \\
\hline${ }^{205} \mathrm{~Pb} /{ }^{204} \mathrm{~Pb}$ & $<5.3 \times 10^{-4}$ & $<5.1 \times 10^{-4}$ & $<3.5 \times 10^{-4}$ \\
\hline \multicolumn{4}{|c|}{$f_{0}=5 \times 10^{-3} ;\left(2.0 M_{\odot}, Z_{\odot}\right)$} \\
\hline$\left(N^{R} / N^{I}\right)_{\Delta_{1}}$ & $\Delta_{1}=0$ & $\Delta_{1}=0.68 \mathrm{Myr}$ & $\Delta_{1}+\Delta_{2}=4.78 \mathrm{Myr}$ \\
\hline${ }^{26} \mathrm{Al} /{ }^{27} \mathrm{Al}\left(1.9 \times 10^{-2}\right)^{*}$ & $\left(9.6 \times 10^{-5}\right)$ & $\left(5.0 \times 10^{-5}\right)$ & $\left(1.0 \times 10^{-6}\right)$ \\
\hline${ }^{36} \mathrm{Cl} /{ }^{35} \mathrm{Cl}$ & $3.6 \times 10^{-6}$ & $7.4 \times 10^{-7}$ & - \\
\hline${ }^{41} \mathrm{Ca} /{ }^{40} \mathrm{Ca}$ & $1.4 \times 10^{-6}$ & $\left(1.5 \times 10^{-8}\right)^{\dagger}$ & - \\
\hline${ }^{60} \mathrm{Fe} /{ }^{56} \mathrm{Fe}$ & $4.2 \times 10^{-8}$ & $3.1 \times 10^{-8}$ & $4.8 \times 10^{-9}$ \\
\hline${ }^{81} \mathrm{Kr} /{ }^{82} \mathrm{Kr}$ & $8.2 \times 10^{-6}$ & $8.6 \times 10^{-7}$ & - \\
\hline${ }^{93} \mathrm{Zr} /{ }^{92} \mathrm{Zr}$ & $3.3 \times 10^{-5}$ & $2.4 \times 10^{-5}$ & $3.7 \times 10^{-6}$ \\
\hline${ }^{99} \mathrm{Tc} /{ }^{100} \mathrm{Ru}$ & $1.6 \times 10^{-5}$ & $1.5 \times 10^{-6}$ & - \\
\hline${ }^{107} \mathrm{Pd} /{ }^{108} \mathrm{Pd}$ & $3.3 \times 10^{-5}$ & $3.1 \times 10^{-5}$ & $\left(2.0 \times 10^{-5}\right)^{* *}$ \\
\hline${ }^{135} \mathrm{Cs} /{ }^{133} \mathrm{Cs}$ & $4.9 \times 10^{-5}$ & $3.9 \times 10^{-5}$ & $9.5 \times 10^{-6}$ \\
\hline${ }^{205} \mathrm{~Pb} /{ }^{204} \mathrm{~Pb}$ & $<3.5 \times 10^{-4}$ & $<3.4 \times 10^{-4}$ & $<2.8 \times 10^{-4}$ \\
\hline
\end{tabular}




\begin{tabular}{|c|c|c|c|}
\hline \multicolumn{4}{|c|}{$f_{0}=5 \times 10^{-3} ;\left(3.0 M_{\odot}, Z_{\odot}\right)$} \\
\hline$\left(N^{R} / N^{I}\right)_{\Delta_{1}}$ & $\Delta_{1}=0$ & $\Delta_{1}=0.70 \mathrm{Myr}$ & $\Delta_{1}+\Delta_{2}=5.1 \mathrm{Myr}$ \\
\hline${ }^{26} \mathrm{Al} /{ }^{27} \mathrm{Al}\left(1.9 \times 10^{-2}\right)$ & $\left(9.7 \times 10^{-5}\right)$ & $\left(5.0 \times 10^{-5}\right)$ & $\left(7.6 \times 10^{-7}\right)$ \\
\hline${ }^{36} \mathrm{Cl} /{ }^{35} \mathrm{Cl}$ & $3.6 \times 10^{-6}$ & $7.1 \times 10^{-7}$ & - \\
\hline${ }^{41} \mathrm{Ca} /{ }^{40} \mathrm{Ca}$ & $1.6 \times 10^{-6}$ & $\left(1.5 \times 10^{-8}\right)^{\dagger}$ & - \\
\hline${ }^{60} \mathrm{Fe} /{ }^{56} \mathrm{Fe}$ & $2.3 \times 10^{-7}$ & $1.7 \times 10^{-7}$ & $2.3 \times 10^{-8}$ \\
\hline${ }^{81} \mathrm{Kr} /{ }^{82} \mathrm{Kr}$ & $6.7 \times 10^{-6}$ & $6.6 \times 10^{-7}$ & - \\
\hline${ }^{93} \mathrm{Zr} /{ }^{92} \mathrm{Zr}$ & $4.6 \times 10^{-5}$ & $3.3 \times 10^{-5}$ & $4.5 \times 10^{-6}$ \\
\hline${ }^{99} \mathrm{Tc} /{ }^{100} \mathrm{Ru}$ & $1.9 \times 10^{-5}$ & $1.7 \times 10^{-6}$ & - \\
\hline${ }^{107} \mathrm{Pd} /{ }^{108} \mathrm{Pd}$ & $3.5 \times 10^{-5}$ & $3.2 \times 10^{-5}$ & $\left(2.0 \times 10^{-5}\right)^{* *}$ \\
\hline${ }^{135} \mathrm{Cs} /{ }^{133} \mathrm{Cs}$ & $8.9 \times 10^{-5}$ & $7.0 \times 10^{-5}$ & $1.5 \times 10^{-5}$ \\
\hline${ }^{205} \mathrm{~Pb} /{ }^{204} \mathrm{~Pb}$ & $<4.8 \times 10^{-4}$ & $<4.6 \times 10^{-4}$ & $<3.8 \times 10^{-4}$ \\
\hline \multicolumn{4}{|c|}{$f_{0}=3.16 \times 10^{-4} ;\left(5.0 M_{\odot}, Z_{\odot}\right)$} \\
\hline$\left(N^{R} / N^{I}\right)_{\Delta_{1}}$ & $\Delta_{1}=0$ & $\Delta_{1}=0.14 \mathrm{Myr}$ & $\Delta_{1}+\Delta_{2}=4.54 \mathrm{Myr}$ \\
\hline${ }^{26} \mathrm{Al} /{ }^{27} \mathrm{Al}(0.18)^{*}$ & $\left(5.7 \times 10^{-5}\right)$ & $\left(5.0 \times 10^{-5}\right)$ & $\left(7.6 \times 10^{-7}\right)$ \\
\hline${ }^{36} \mathrm{Cl} /{ }^{35} \mathrm{Cl}$ & $3.3 \times 10^{-7}$ & $2.4 \times 10^{-7}$ & - \\
\hline${ }^{41} \mathrm{Ca} /{ }^{40} \mathrm{Ca}$ & $3.7 \times 10^{-8}$ & $\left(1.5 \times 10^{-8}\right)^{\dagger}$ & - \\
\hline${ }^{60} \mathrm{Fe} /{ }^{56} \mathrm{Fe}$ & $1.2 \times 10^{-6}$ & $1.1 \times 10^{-6}$ & $1.5 \times 10^{-7}$ \\
\hline${ }^{81} \mathrm{Kr} /{ }^{82} \mathrm{Kr}$ & $1.8 \times 10^{-5}$ & $1.1 \times 10^{-5}$ & - \\
\hline${ }^{93} \mathrm{Zr} /{ }^{92} \mathrm{Zr}$ & $2.6 \times 10^{-4}$ & $2.4 \times 10^{-4}$ & $3.2 \times 10^{-5}$ \\
\hline${ }^{99} \mathrm{Tc} /{ }^{100} \mathrm{Ru}$ & $6.4 \times 10^{-5}$ & $4.1 \times 10^{-5}$ & - \\
\hline${ }^{107} \mathrm{Pd} /{ }^{108} \mathrm{Pd}$ & $3.2 \times 10^{-5}$ & $3.2 \times 10^{-5}$ & $\left(2.0 \times 10^{-5}\right)^{* *}$ \\
\hline${ }^{135} \mathrm{Cs} /{ }^{133} \mathrm{Cs}$ & $2.8 \times 10^{-5}$ & $2.6 \times 10^{-5}$ & $5.8 \times 10^{-6}$ \\
\hline${ }^{205} \mathrm{~Pb} /{ }^{204} \mathrm{~Pb}$ & $<5.6 \times 10^{-5}$ & $<5.6 \times 10^{-5}$ & $<4.6 \times 10^{-5}$ \\
\hline
\end{tabular}

\footnotetext{
* For all of Table 5 for each model this is the value required in the envelope to give $\left({ }^{26} \mathrm{Al} /{ }^{27} \mathrm{Al}\right)=5 \times 10^{-5}$ at the chosen $\Delta_{1}$.

$\dagger$ This is the value assumed to determine $\Delta_{1}$.

** Value used to determine $f_{0}$ for a selected $\Delta_{1}$. All values that are assumed are shown in parenthesis.
} 
Table 6

Abundances of short-lived nuclei in a cloud salted with ejecta from a $3 M_{\odot}$ AGB star with $Z / Z_{\odot}=1 / 3\left(\right.$ no $\left.{ }^{13} \mathrm{C}\right)$

\begin{tabular}{|c|c|c|c|c|}
\hline \multicolumn{5}{|c|}{$f_{0}=4 \times 10^{-3} ;\left(3 M_{\odot}, Z_{\odot}=1 / 3 Z_{\odot}\right)^{\mathrm{a}}$} \\
\hline & $\left(N^{R} / N^{I}\right)_{E N V}\left(q_{E N V}^{I} / q_{0}^{I}\right)^{\mathrm{b}}$ & \multicolumn{3}{|c|}{$\left(N^{R} / N^{I}\right)_{\Delta}$} \\
\cline { 3 - 5 } & & $\Delta_{1}=0 \mathrm{Myr}$ & $\Delta_{1}=0.55 \mathrm{Myr}$ & $\Delta_{1}=6.7 \mathrm{Myr}$ \\
\hline${ }^{26} \mathrm{Al} /{ }^{27} \mathrm{Al}$ & $\left(2.0 \times 10^{-2}\right)$ & $\left(8.0 \times 10^{-5}\right)$ & $\left(5.0 \times 10^{-5}\right)$ & $\left(8.5 \times 10^{-8}\right)$ \\
\hline${ }^{36} \mathrm{Cl} /{ }^{35} \mathrm{Cl}$ & $4.4 \times 10^{-4}$ & $1.7 \times 10^{-6}$ & $4.7 \times 10^{-7}$ & - \\
\hline${ }^{41} \mathrm{Ca} /{ }^{40} \mathrm{Ca}$ & $1.5 \times 10^{-4}$ & $5.9 \times 10^{-7}$ & $\left(1.5 \times 10^{-8}\right)$ & - \\
\hline${ }^{60} \mathrm{Fe} /{ }^{56} \mathrm{Fe}$ & $6.7 \times 10^{-4}$ & $2.7 \times 10^{-6}$ & $2.1 \times 10^{-6}$ & $1.0 \times 10^{-7}$ \\
\hline${ }^{81} \mathrm{Kr} /{ }^{82} \mathrm{Kr}$ & $1.6 \times 10^{-2}$ & $6.4 \times 10^{-5}$ & $1.0 \times 10^{-5}$ & \\
\hline${ }^{93} \mathrm{Zr} /{ }^{2} \mathrm{Zr}$ & $8.1 \times 10^{-2}$ & $3.2 \times 10^{-4}$ & $2.5 \times 10^{-4}$ & $1.2 \times 10^{-5}$ \\
\hline${ }^{99} \mathrm{Tc} /{ }^{100} \mathrm{Ru}$ & $2.6 \times 10^{-1}$ & $1.1 \times 10^{-3}$ & $1.9 \times 10^{-5}$ & - \\
\hline${ }^{107} \mathrm{Pd} /{ }^{108} \mathrm{Pd}$ & $9.9 \times 10^{-3}$ & $4.1 \times 10^{-5}$ & $3.8 \times 10^{-5}$ & $\left(2.0 \times 10^{-5}\right)$ \\
\hline${ }^{135} \mathrm{Cs} /{ }^{133} \mathrm{Cs}$ & $1.1 \times 10^{-2}$ & $4.4 \times 10^{-5}$ & $3.6 \times 10^{-5}$ & $3.5 \times 10^{-6}$ \\
\hline${ }^{205} \mathrm{~Pb} /{ }^{204} \mathrm{~Pb}$ & $<8.6 \times 10^{-2}$ & $<3.5 \times 10^{-4}$ & $<3.4 \times 10^{-4}$ & $<2.5 \times 10^{-4}$ \\
\hline
\end{tabular}

a Calculated to match $\left({ }^{26} \mathrm{Al} /{ }^{27} \mathrm{Al}\right)_{0},\left({ }^{41} \mathrm{Ca} /{ }^{40} \mathrm{Ca}\right)_{0.55 \mathrm{Myr}},\left({ }^{107} \mathrm{Pd} /{ }^{108} \mathrm{Pd}\right)_{6.7 \mathrm{Myr}}$.

b Values in the envelope calculated for $Z=1 / 3 Z_{\odot}$ with the factor of $q_{E N V}^{I}$ for this $Z$ divided by $\left(q_{0}^{I}\right)_{\odot}$ for the unsalted protosolar cloud. 
Table 7

Possible Sources of Short-Lived Nuclei

Requiring Late Addition $(\leq 10 \mathrm{Myr})$

\begin{tabular}{|c|c|c|}
\hline NUCLIDE & POSSIBLE & EXCLUDED \\
\hline${ }^{10} \mathrm{Be}$ & IRRAD & $\mathrm{AGB}, \mathrm{SNe}$ \\
\hline${ }^{26} \mathrm{Al}$ & AGB, IRRAD. & $\mathrm{SNe}$ \\
\hline${ }^{36} \mathrm{Cl}$ & AGB?, IRRAD. & $\mathrm{SNe}$ \\
\hline${ }^{41} \mathrm{Ca}$ & AGB, IRRAD. & $\mathrm{SNe}$ \\
\hline${ }^{53} \mathrm{Mn}$ & SNe, IRRAD. & AGB \\
\hline${ }^{60} \mathrm{Fe}$ & $\mathrm{AGB}, \mathrm{SNe}$ & IRRAD. \\
\hline${ }^{107} \mathrm{Pd}$ & AGB & SNe, IRRAD. \\
\hline${ }^{135} \mathrm{Cs} ?$ & AGB & SNe, IRRAD. \\
\hline${ }^{205} \mathrm{~Pb} ?$ & AGB & SNe, IRRAD. \\
\hline
\end{tabular}




\section{References}

[1] J. H. Reynolds, Phys. Rev. Lett. 4 (1960) 8.

[2] P. M. Jeffery and J. H. Reynolds, J. G. R. 66 (1961) 3582.

[3] G. J. Wasserburg, W. A. Fowler and F. Hoyle, Phys. Rev. Lett. 4 (1960) 112.

[4] L. Grossman, Geochim. Cosmochim. Acta 36 (1972) 597.

[5] L. Grossman, Annu. Rev. Earth \& Planet. Sci. 8 (1980) 559.

[6] U. B. Marvin, J. A. Wood and J. S. Dickey, Earth Planet. Sci. Lett. 7 (1970) 346.

[7] F. A. Podosek and R. S. Lewis, Earth Planet. Sci. Lett. 15 (1972) 101.

[8] D. N. Schramm, F. Tera and G. J. Wasserburg, Earth Planet. Sci. Lett. 10 (1970) 44.

[9] R. N. Clayton, L. Grossman and T. K. Mayeda, Science 182 (1973) 485.

[10] C. M. Gray, D. A. Papanastassiou and G. J. Wasserburg, Icarus 20 (1973) 213.

[11] C. M. Gray and W. Compston, Nature 251 (1976) 495.

[12] T. Lee and D. A. Papanastassiou, Geophys. Res. Lett. 1 (1974) 225.

[13] T. Lee, D. A. Papanastassiou and G. J. Wasserburg, Geophys. Res. Lett. 3 (1976) 109.

[14] T. Lee, D. A. Papanastassiou and G. J. Wasserburg, Ap. J. 211 (1976) L107.

[15] H. C. Urey, Proc. Nat. Acad. Sci. US. 41 (1955) 127.

[16] H. C. Urey and B. Donn, Ap. J. 124 (1956) 307.

[17] J. R. Simanton, R. A. Rightmire, A. L. Long and T. P. Kohman, Phys. Rev. 96 (1954) 1711.

[18] M. W. Rowe and P. K. Kuroda, J. Geophys. Res. 70 (1965) 709.

[19] Y. Cantelaube, H. Maurette and P. Pellas, in Radioactive Dating Methods and Low Level Counting (Atomic Energy: Vienna, 1967) 215.

[20] G. J. Wasserburg, J. C. Huneke and D. S. Burnett, J. Geophys. Res. 74 (1969) 4221.

[21] G. J. Wasserburg, J. C. Huneke and D. S. Burnett, Phys. Rev. Lett. 22 (1969) 1198.

[22] D. Storzer and P. Pellas, Earth Planet. Sci. Lett. 35 (1977) 285.

[23] E. C. Alexander, Jr., R. S. Lewis, J. H. Reynolds and M. C. Michel, Science 172 (1971) 837. 
[24] G. W. Lugmair and K. Marti, Earth Planet. Sci. Lett. 35 (1977) 273.

[25] J. Audouze and D. N. Schramm, Nature 237 (1972) 447.

[26] S. B. Jacobsen and G. J. Wasserburg, Earth Planet. Sci. Lett. 67 (1984) 137.

[27] A. Prinzhofer, D. A. Papanastassiou and G. J. Wasserburg, Ap. J. 344 (1989) L81.

[28] B. W. Stewart, D. A. Papanastassiou and G. J. Wasserburg, Geochim. Cosmochim. Acta 58 (1994) 3487.

[29] C. L. Harper Jr., Ap. J. 466 (1996) 437.

[30] W. R. Kelly and G. J. Wasserburg, Geophys. Res. Lett. 5 (1978) 1079.

[31] J. H. Chen and G. J. Wasserburg, Geochim. Cosmochim. Acta 47 (1983) 1725.

[32] J. H. Chen and G. J. Wasserburg, in Geophys. Monograph 95, Earth Processes: Reading the Isotopic Code, ed. A. Basu and S. Hart, (Am. Geophys. Union: Washington DC, 1996) 1-20.

[33] R. W. Carlson and E. Hauri, Geochim. Cosmochim. Acta 65 (2001) 1839.

[34] C. L. Harper Jr. and S. B. Jacobsen, in Geophys. Monogr. 95, Earth Processes: Reading the Isotopic Code, ed. A. Basu, S. Hart, (Am. Geophys. Union: Washington DC, 1996) 77.

[35] C. L. Harper Jr. and S. B. Jacobsen, Geochim. Cosmochim. Acta 60 (1996) 1131.

[36] D.-C. Lee and A. N. Halliday, Nature 378 (1995) 771.

[37] D.-C. Lee and A. N. Halliday, Science 274 (1996) 1876.

[38] J.-L. Birck and C. A. Allègre, Geophys. Res. Lett. 12 (1985) 745.

[39] J.-L. Birck and C. A. Allègre, Nature 331 (1988) 579.

[40] I. D. Hutcheon, A. N. Krot, K. Keil, D. L. Phinney and E. R. D. Scott, Science 282 (1998) 1865.

[41] G. W. Lugmair and A. Shukolyukov, Geochim. Cosmochim. Acta 62 (1998) 2863.

[42] A. Shukolyukov and G. W. Lugmair, Science 259 (1993) 1138.

[43] A. Shukolyukov and G. W. Lugmair, Earth Planet. Sci. Lett. 119 (1993) 159.

[44] S. Tachibana and G. R. Huss, Ap. J. 588 (2003) L41.

[45] S. Mostefaoui, G. W. Lugmair, P. Hoppe and A. El Goresy, Lunar Planet. Sci. 34 (2003) 1585.

[46] S. Mostefaoui, G. W. Lugmair, P. Hoppe and A. El Goresy, New Astron. Rev. 48 (2004) 155. 
[47] J. H. Chen and G. J. Wasserburg, Lunar Planet. Sci. 18 (1987) 165.

[48] G. Srinivasan, A. A. Ulyanov, and J. N. Goswami, Ap. J. 431 (1994) L67.

[49] G. Srinivasan, S. Sahijpal, A. A. Ulyanov, and J. N. Goswami, Geochim. Cosmochim. Acta 60 (1996) 1823.

[50] K. D. McKeegan, M. Chaussidon and F. Robert, Science 289 (2000) 1334.

[51] D. C. Black, Geochim. Cosmochim. Acta 36 (1972) 377.

[52] E. Anders and E. Zinner, Meteoritics 28 (1993) 490.

[53] E. Anders and E. Zinner, Icarus 112 (1994) 303.

[54] B. T. Draine, in Origin and Distribution of the Elements, Vol. 4, (2004) Carnegie Observatories Astrophysics Series, ed. A. McWilliam and M. Rauch (Cambridge Univ. Press: Cambridge, UK, 2004)

[55] B. T. Draine, in The Cold Universe, Saas-Fee Advanced Course 32, ed. D. Pfenniger (Springer Verlag: Berlin, 2004) 213.

[56] B. T. Draine, Annu. Rev. Astron. Astrophys. 41 (2003) 241.

[57] K. D. McKeegan, A. M. Davis, in Treatise on Geochemistry, Vol. I, eds. A. M. Davis, H. D. Holland, K. K. Turekian (Elsevier-Pergamon: Oxford, 2004) p. 431.

[58] M. Busso, R. Gallino and G. J. Wasserburg, Publ. Astro. Soc. Australia 20 (2003) 356 .

[59] D. D. Clayton and L. R. Nittler, Annu. Rev. Astron. Astrophys. 42 (2004) 39.

[60] E. Zinner, S. Amari, E. Anders and R. S. Lewis, Nature 349 (1991) 51.

[61] K. M. Nollett, M. Busso and G. J. Wasserburg, Ap. J. 582 (2003) 1036.

[62] G. K. Nicolussi, M. J. Pellin, A. M. Davis et al., Geochim. Cosmochim. Acta 62 (1998) 1093.

[63] G. K. Nicolussi, M. J. Pellin, K. R. Lykke, J. L. Trevor, D. E. Mencer and A. M. Davis, Surface and Interface Analysis 24 (1996) 363.

[64] G. K. Nicolussi, M. J. Pellin, W. F. Calaway, R. S. Lewis, A. M. Davis, S. Amari and R. N. Clayton, Anal. Chem. 69 (1997) 1140.

[65] K. K. Marhas, P. Hoppe, U. Ott, Meteorit. Planet. Sci. Suppl. 39 (2004) 5121.

[66] M. R. Savina, A. M. Davis, C. E. Tripa, M. J. Pellin, R. Gallino, R. S. Lewis and S. Amari, Science 303 (2004) 649.

[67] G. R. Huss, I. D. Hutcheon, G. J. Wasserburg and J. Stone, Lunar Planet. Sci. 23 (1992) 563.

[68] G. R. Huss, A. J. Fahey, R. Gallino and G. J. Wasserburg, Ap. J. 430 (1994) L81. 
[69] I. D. Hutcheon, G. R. Huss, A. J. Fahey and G. J. Wasserburg, Ap. J. 425 (1994) L97.

[70] L. R. Nittler, C. M. O’D. Alexander, X. Gao, R. M. Walker and E. K. Zinner, Nature 370 (1994) 443.

[71] L. R. Nittler, C. M. O'D. Alexander, X. Gao, R. M. Walker and E. Zinner, Ap. J. 483 (1997) 475.

[72] L. R. Nittler, C. M. O’D. Alexander, J. Wang and X. Gao, Nature 393 (1998) 222 .

[73] D. S. P. Dearborn, Phys. Rep. 210 (1992) 367.

[74] B.-G. Choi, G. J. Wasserburg and G. R. Huss, Ap. J. 522 (1999) L133.

[75] L. R. Nittler, C. M. O’D. Alexander, F. J. Stadermann and E. K. Zinner, Lunar Planet. Sci. XXXVI (2005) 2200.

[76] A. N. Nguyen, E. Zinner, Science 303 (2004) 1496.

[77] A. N. Nguyen, E. Zinner, R. M. Stroud, Lunar Planet. Sci. XXXVI (2005) 2196.

[78] P. Hoppe, S. Mostefaoui and T. Stephan, Lunar Planet. Sci. XXXVI (2005) 1301.

[79] S. Mostefaoui, K. K. Marhas, P. Hoppe, Lunar Planet. Sci. XXXV (2004) 1593.

[80] S. Messenger, L.P. Keller, F. J. Staderman, R. M. Walker, E. Zinner, Science $300(2003) 105$.

[81] F. J. Stadermann, T. K. Croat, T. J. Bernatowicz, S. Amari, S. Messenger, R. M. Walker and E. Zinner, Geochim. Cosmochim. Acta 69 (2005) 177.

[82] B.-G. Choi, G. R. Huss, G. J. Wasserburg and R. Gallino, Science 282 (1998) 1284 .

[83] O. Straniero, R. Gallino, S. Cristallo, Nucl. Phys. A. (2005) in press.

[84] M. Busso, R. Gallino and G. J. Wasserburg, Annu. Rev. Astron. Astrophys. 37 (1999) 239.

[85] I. Iben, Jr. and A. Renzini, Annu. Rev. Astron. Astrophys. 21 (1983) 271.

[86] O. Straniero, R. Gallino, M. Busso, A. Chieffi, C.M. Raiteri, M. Limongi, M. Salaris, Ap. J. 440 (1995) L85.

[87] C. A. Frost, J. C. Lattanzio Ap. J. 473 (1996) 383.

[88] A. I. Karakas, J. C. Lattanzio, Publ. Astron. Soc. Australia 20 (2003) 393.

[89] O. Straniero, I. Dominguez, S. Cristallo, R. Gallino, Publ. Astron. Soc. Australia 20 (2003) 389.

[90] A. G. W. Cameron, A. J. 65 (1960) 485. 
[91] A. G. W. Cameron, A. J. 61 (1956) 2.

[92] A. G. W. Cameron, Chalk River Rep. CRL-41 (1957).

[93] J. R. Greenstein, Modern Physics for Engineers, ed. L. Ridenour (McGrawHill: New York, 1954).

[94] M. Lugaro, F. Herwig, J.C. Lattanzio, R. Gallino, O. Straniero, Ap. J. 586 (2003) 1305.

[95] C. Travaglio, D. Galli, R. Gallino et al., Ap. J. 521 (1999) 691.

[96] C. Arlandini, F. Käppeler, K. Wisshak et al., Ap. J. 525 (1999) 886.

[97] G. J. Wasserburg, M. Busso, R. Gallino and C. M. Raiteri, Ap. J. 424 (1994) 412.

[98] C. Charbonnel, Ap. J. 453 (1995) L41.

[99] C. Charbonnel, in Origin and Evolution of the Elements, Ed. A. McWilliam and M. Rauch (Cambridge University Press: Cambridge, UK, 2004) 60.

[100] F. Herwig, Annu. Rev. Astron. Astrophys. 43 (2005) in press.

[101] K. K. Gilroy and J. A. Brown, Ap. J. 371 (1991) 578.

[102] A. I. Boothroyd and I.-J. Sackmann, Ap. J. 510 (1999) 232.

[103] S. Lucatello, R. Gratton, J. G. Cohen et al. A. J. 125 (2003) 875.

[104] G. J. Wasserburg, A. I. Boothroyd and I.-J. Sackmann, Ap. J. 447 (1995) L37.

[105] B. Messenger, PhD thesis, Monash University at Clayton, School of Mathematics and Statistics (Australia) (2000).

[106] P. A. Denissenkov and A. Weiss, Astron. Astrophys. 308 (1996) 773.

[107] P. A. Denissenkov, G. S. Da Costa, J. E. Norris and A. Weiss, Astron. Astrophys. 333 (1998) 926.

[108] A. Weiss, P. A. Denissenkov and C. Charbonnel, Astron. Astrophys. 356 (2000) 181.

[109] C. A. Frost, R. C. Cannon, J. C. Lattanzio, P. R. Wood and M. Forestini, Astron. Astrophys. 332 (1998) L17.

[110] J. C. Lattanzio and M. Forestini, in Asymptotic Giant Branch Stars, IAU Symposium 191, ed. T. Le Bertre, A. Lèbre and C. Waelkens, (Astronomical Society of the Pacific: San Francisco, 1999) 227.

[111] J. Lattanzio, M. Forestini and C. Charbonnel, Memoria Soc. Astron. Italiana $71(2000) 737$.

[112] C. Angulo, M. Arnould, M. Rayet et al., Nucl. Phys. A 656 (1999) 3.

[113] C. Fox, C. Iliadis, A. E. Champagne et al., Phys. Rev. Lett. 93 (2004) 081102. 
[114] D. Péquignot, J. R. Walsh, A. A. Zijlstra and G. Dudziak, Astron. Astrophys. 361 (2000) L1.

[115] C. M. Sharp and G. J. Wasserburg, Geochim. Cosmochim. Acta 59 (1995) 1633.

[116] K. Lodders and B. Fegley Jr., Meteoritics 30 (1995) 661.

[117] Y.-N. Chin, C. Henkel, N. Langer and R. Mauersberger, Ap. J. 512 (1999) L143.

[118] T. L. Wilson and R. T. Rood, Annu. Rev. Astron. Astrophys. 32 (1994) 191.

[119] A. Formicola, G. Imbriani, H. Costantini et al. Phys. Lett. B 591 (2004) 61.

[120] R. C. Runkle, A. E. Champagne, C. Fox, C. Iliadis, J. Pollanen, A. Stephan, C. Westerfeldt, Phys. Rev. C. 66 (2002) 022801.

[121] R. C. Runkle, A. E. Champagne, C. Angulo, C. Fox, C. Iliadis, R. Longland, J. Pollanen, Phys. Rev. Lett. 94 (2005) 082503.

[122] G. R. Huss, I. D. Hutcheon and G. J. Wasserburg, Geochim. Cosmochim. Acta 61 (1997) 5117.

[123] A. I. Karakas, J. Lattanzio, Publ. Astron. Soc. Australia 20 (2003) 279.

[124] A. Renzini, M. Voli, Astron. Astrophys. 94 (1981) 175.

[125] A. I. Karakas, Ph.D. thesis, Monash University at Clayton, School of Mathematics and Statistics (Australia) (2003).

[126] L. R. Nittler, Earth Planet. Sci. Lett. 209 (2003) 259.

[127] D. N. Schramm and G. J. Wasserburg, Ap. J. 162 (1970) 57.

[128] G. J. Wasserburg and D. A. Papanastassiou, in Essays in Nuclear Astrophysics, ed. C. A. Barnes, D. D. Clayton and D. N. Schramm (Cambridge Univ. Press: New York, 1982) 77.

[129] E. M. Burbidge, G. R. Burbidge, W. A. Fowler and F. Hoyle, Rev. Mod. Phys. $29(1957) 547$.

[130] W. A. Fowler and F. Hoyle, Ann. Phys. 10 (1960) 280.

[131] G. J. Wasserburg, M. Busso and R. Gallino, Ap. J. 466 (1996) L109.

[132] R. Diehl, M. Cerviño, D. H. Hartmann and K. Kretschner, New Astro. Rev. 48 (2004) 81.

[133] S. E. Woosley and T. A. Weaver, Ap. J. Suppl. Ser. 101 (1995) 181.

[134] T. Rauscher, A. Heger, R. D. Hoffmann and S. E. Woosley, Ap. J. 576 (2002) 323.

[135] U. Ott, Science 288 (2000) 1761. 
[136] J. Whitby, R. Burgess, G. Turner et al., Science 288 (2000) 1819.

[137] A. G. W. Cameron, in Protostars and Planets III, ed. E. H. Levy and J. I. Lunine, (Univ. Arizona Press: Tucson, 1993) 47.

[138] A. G. W. Cameron, F.-K. Thielemann and J. J. Cowan, Phys. Rep. 227 (1993) 283.

[139] F. Käppeler, H. Beer and K. Wisshak, Rep. Prog. Phys. 52 (1989) 945.

[140] Q. Yin, S. B. Jacobsen, K. Yamashita, J. Blichert-Toft, P. Télouk, F. Albarède, Nature 418 (2002) 949.

[141] T. Kleine, C. Münker, K. Mezger and H. Palme, Nature 418 (2002) 952.

[142] A. McWilliam, G. W. Preston, C. Sneden and L. Searle, A. J. 109 (1995) 2757.

[143] A. McWilliam, A. J. 115 (1998) 1640.

[144] C. Sneden, J. J. Cowan, J. E. Lawler et al., Ap. J. 591 (2003) 936.

[145] V. Hill, B. Plez, R. Cayrel et al., Astron. Astrophys. 387 (2002) 560.

[146] C. Sneden, J. J. Cowan, I. I. Ivans et al., Ap. J. 533 (2000) L139.

[147] Y.-Z. Qian and G. J. Wasserburg, Ap. J. 567 (2002) 515.

[148] Y.-Z. Qian and G. J. Wasserburg, Ap. J. 588 (2003) 1099.

[149] B. S. Meyer, Phys. Rev. Lett. 89 (2002) 231101.

[150] T. A. Thompson, A. Burrows and B. S. Meyer, Ap. J. 562 (2001) 887.

[151] D. Argast, M. Samland, F.-K. Thielemann, Y.-Z. Qian, Astron. Astrophys. 416 (2004) 997.

[152] S. Honda, W. Aoki, T. Kajino et al., Ap. J. 604 (2004) 474.

[153] K.-L. Kratz, B. Pfeiffer, J. J. Cowan and C. Sneden, New Astron. Rev. 48 (2004) 105.

[154] J. C. Niemeyer, J. W. Truran, (eds.), "Type Ia Supernovae, Theory and Cosmology" Cambridge Contemporary Astrophysics Series, (Cambridge Univ. Press: Cambridge, UK, 2000).

[155] Y.-Z. Qian, Prog. in Part. Nucl. Phys. 50 (2003) 153.

[156] Y.-Z. Qian, Ap. J. 569 (2002) L103.

[157] J. H. Chen and G. J. Wasserburg, Anal. Chem. 53 (1981) 2060.

[158] J. H. Chen and G. J. Wasserburg, Earth Planet. Sci. Lett. 52 (1981) 1.

[159] C. H. Stirling, A. N. Halliday, D. Porcelli, Geochim. Cosmochim. Acta 69 (2005) 1059.

[160] G. R. Huss and R. S. Lewis, Geochim. Cosmochim. Acta 59 (1995) 115. 
[161] D. S. Ebel and L. Grossman, Geochim. Cosmochim. Acta 64 (2000) 339.

[162] M. Blander, A. D. Pelton, I.-H. Jung, and R. Weber, Meteorit. Planet. Sci. 39 (2004) 1897.

[163] P. Cassen, Meteorit. Planet. Sci. 36 (2001) 671.

[164] F. H. Shu, H. Shang and T. Lee, Science 271 (1996) 1545.

[165] J. A. Wood, Earth Planet. Sci. Lett. 70 (1984) 11.

[166] J. N. Cuzzi, Icarus 168 (2004) 484.

[167] H. C. Connolly, Jr., S. G. Love, Science 280 (1998) 62.

[168] J. N. Cuzzi, R. C. Hogan, Icarus 164 (2003) 127.

[169] A. P. Boss, R. H. Durisen, Ap. J. 621 (2005) L137.

[170] Y. Amelin, A. N. Krot, I. D. Hutcheon and A. A. Ulyanov, Science 297 (2002) 1678.

[171] C. Göpel, G. Manhès and C. J. Allègre, Earth Planet. Sci. Lett. 121 (1994) 153.

[172] E. Zinner, C. Göpel, Meteorit. Planet. Sci. 37 (2002) 1001.

[173] Y. Amelin, A. Ghosh and E. Rothenberg, Geochim. Cosmochim. Acta 69 (2005) 505.

[174] F. Tera, R. W. Carlson, Geochim. Cosmochim. Acta 63 (1999) 1877.

[175] F. Tera, R. W. Carlson, N. Z. Boctor, Geochim. Cosmochim. Acta 61 (1997) 1713.

[176] G. J. Wasserburg, R. Gallino and M. Busso, Ap. J. 500 (1998) L189.

[177] F. X. Timmes, S. E. Woosley, D. H. Hartmann et al., Ap. J. 449 (1995) 204.

[178] T. Nakamura, H. Umeda, K. Nomoto, F.-K. Thielemann, A. Burrows, 1999, Ap. J. 517 (1999) 193.

[179] M. Limongi, A. Chieffi Ap. J. 592 (2003) 404.

[180] D. M. Smith, New Astron. Rev 48 (2004) 86.

[181] M. J. Harris, J. Knödlseder, P. Jean et al., Astron. Astrophys. 433 (2005) L49.

[182] N. Prantzos, Astron. Astrophys. 420 (2004) 1033.

[183] M. Thiemens, J. E. Heidenreich III, Science 219 (1983) 1073.

[184] K. Mauersberger, B. Erbacher, D. Krankowsky, J. Günther and R. Nickel, Science 283 (1999) 370.

[185] K. Mauersberger, Geophys. Res. Lett. 8 (1981) 935. 
[186] Y. Q. Gao, W.-C. Chen and R. A. Marcus, J. Chem. Phys. 117 (2002) 1536.

[187] R. A. Marcus, J. Chem. Phys. 121 (2004) 8201.

[188] O. Navon and G. J. Wasserburg, Earth Planet. Sci. Lett. 73 (1985) 1.

[189] R. N. Clayton, Nature 415 (2002) 860.

[190] H. Yurimoto, K. Kuramoto, Science 305 (2004) 1763.

[191] R. N. Clayton, Lunar Planet. Sci. XXXVI (2005) 1711.

[192] S. Chakraborty and M. Thiemens, Lunar Planet. Sci. XXXVI (2005) 1113.

[193] J. R. Lyons and E. D. Young, Nature 435 (2005) 317.

[194] L. Andric, F. Bouakline, T. P. Grozdanov and R. McCarroll, Astron. Astrophys. 421 (2004) 381.

[195] K. Hashizume, M. Chaussidon, Nature 434 (2005) 619.

[196] A. N. Krot, I. D. Hutcheon, H. Yurimoto et al., Ap. J. 622 (2005) 1333.

[197] P. N. Foster and A. P. Boss, Ap. J. 468 (1996) 784.

[198] B. S. Meyer and D. D. Clayton, Space Sci. Rev. 92 (2000) 133.

[199] S. Tachibana, G. R. Huss, N. T. Kita, H. Shimoda, Y. Morishita, Lunar Planet. Sci. 36 (2005) 1529.

[200] K. Nomoto, M. Hashimoto, T. Tsujimoto et al., Nucl. Phys. A616 (1997) 79.

[201] R. Gallino, M. Busso, G. J. Wasserburg, O. Straniero New Astr. Rev. 48 (2004) 133.

[202] Z. Y. Bao, H. Beer, F. Käppeler et al., Atom. Data Nucl. Data Tables 76 (2000) 70 .

[203] M. Lugaro, A. Karakas, A. Champagne, J. Lattanzio and R. Cannon, Mem. Soc. Astron. It. 72 (2001) 319.

[204] R. Gallino, C. Arlandini, M. Busso et al., Ap. J. 497 (1998) 388.

[205] M. Busso, R. Gallino, D. L. Lambert, C. Travaglio and V. V. Smith, Ap. J. 557 (2001) 802.

[206] C. Abia, M. Busso, R. Gallino, I. Domínguez, O. Straniero, J. Isern, Ap. J. 559 (2001) 1117.

[207] C. Abia, I. Domínguez, R. Gallino, M. Busso, S. Masera, O. Straniero, P. de Laverny, B. Plez, J. Isern, Ap. J. 579 (2002) 817.

[208] S. Amari, L. R. Nittler, E. Zinner, R. Gallino, M. Lugaro, R. S. Lewis, Ap. J. 546 (2001) 248.

[209] C. Travaglio, R. Gallino, M. Busso, R. Gratton, Ap. J. 549 (2001) 346. 
[210] S. J. Woodland, M. Rehkämper, D.-C. Lee and A. N. Halliday, Lunar Planet. Sci. 34 (2003) 1621.

[211] G. Srinivasan, J. N. Goswami and N. Bhandari, Science 284 (1999) 1348.

[212] K. Takahashi and K. Yokoi, Atomic Data Nucl. Data Tables 36 (1987) 375.

[213] N. Mowlavi, S. Goriely and M. Arnould, Astron. Astrophys. 330 (1998) 206.

[214] J.-L. Birck and G. W. Lugmair, Earth Planet. Sci. Lett. 90 (1988) 131.

[215] J. Völkening and D. A. Papanastassiou, Ap. J. 347 (1989) L43.

[216] B.-G. Choi, G. R. Huss, G. J. Wasserburg, Lunar Planet. Sci. XXX (1999) 1862.

[217] N. T. Kita, H. Nagahara, S. Togashi and Y. Morishita, Geochim. Cosmochim. Acta 64 (2000) 3913.

[218] G. R. Huss and S. Tachibana, Lunar Planet. Sci. 35 (2004) 1811.

[219] S. Mostefaoui, G. W. Lugmair and P. Hoppe, Lunar Planet. Sci. 35 (2004) 1271.

[220] S. E. Woosley, W. A. Fowler, J. A. Holmes and B. A. Zimmerman, Atomic Data Nucl. Data Tables 22 (1978) 371.

[221] T. Rauscher and F.-K. Thielemann, Atomic Data Nucl. Data Tables 75 (2000) 1.

[222] M. Rotaru, J. L. Birck and C. J. Allègre, Nature 358 (1992) 465.

[223] D. A. Papanastassiou, G. J. Wasserburg and O. Bogdanovski Lunar Planet. Sci. 36 (2005) 2198.

[224] L. Nyquist, D. Lindstrom, D. Mittlefehldt, C.-Y. Shih, H. Wiesmann, S. Wentworth, R. Martinez, Meteorit. Planet. Sci. 36 (2001) 911.

[225] A. J. Brearley, I. D. Hutcheon, Meteorit. Planet. Sci. 37 (2002) A23.

[226] M. Wadhwa and G. W. Lugmair, Geochim. Cosmochim. Acta 60 (1996) 4889.

[227] W. Stegmann and F. Begemann, Earth Planet. Sci. Lett. 55 (1981) 266.

[228] G. J. MacPherson, A. M. Davis, E. K. Zinner, Meteoritics 30 (1995) 365.

[229] R. W. Hinton, and A. Bischoff, Nature 308 (1984) 169.

[230] G. R. Huss, G. J. MacPherson, J. Glenn et al., Meteorit. Planet. Sci. 36 (2001) 975.

[231] Y. Guan, G. R. Huss, G. J. MacPherson and G. J. Wasserburg, Science 289 (2000) 1330.

[232] I. D. Hutcheon and R. Hutchison, Nature 337 (1989) 238. 
[233] Y. J. Sheng, I. D. Hutcheon and G. J. Wasserburg, Geochim. Cosmochim. Acta 55 (1991) 581.

[234] S. S. Russell, G. Srinivasan, G. R. Huss, G. J. Wasserburg and G. J. MacPherson, Science 273 (1996) 757.

[235] A. Galy, E. D. Young, R. D. Ash and R. K. O’Nions, Science 290 (2000) 1751.

[236] M. Bizzarro, J. A. Baker and H. Haack, Nature 431 (2004) 275.

[237] G. Srinivasan, D. A. Papanastassiou, G. J. Wasserburg, N. Bhandari and J. N. Goswami, Lunar Planet. Sci. 31 (2000) 1795.

[238] L. E. Nyquist, Y. Reese, H. Wiesmann, C.-Y. Shih and H. Takeda, Earth Planet. Sci. Lett. 214 (2003) 11.

[239] M. Wadhwa, Y. Amelin, O. Bogdanovski, A. Shukolyukov and G. W. lugmair, Lunar Planet. Sci. 36 (2005) 2126.

[240] A. El Goresy, J. T. Armstrong and G. J. Wasserburg, Geochim. Cosmochim. Acta 49 (1985) 2433.

[241] W. Hsu, G. J. Wasserburg and G. R. Huss, Earth Planet. Sci. Lett. 182 (2000) 15.

[242] A. Galy, I. D. Hutcheon, L. Grossman, Lunar Planet. Sci. XXXV (2004) 1790.

[243] M.-C. Liu, Y. Iizuka, K. D. McKeegan et al., Lunar Planet. Sci. 36 (2005) 2079 .

[244] E. D. Young, J. I. Simon, A. Galy et al., Science 308 (2005) 223.

[245] I. D. Hutcheon, J. T. Armstrong and G. J. Wasserburg, Meteoritics 19 (1984) 243.

[246] S. Sahijpal, J. N. Goswami, A. M. Davis, L. Grossman and R. S. Lewis, Nature 391 (1998) 559.

[247] R. Göbel, F. Begemann and U. Ott, Geochim. Cosmochim. Acta 46 (1982) 1777.

[248] I. M. Villa, J. C. Huneke, D. A. Papanastassiou, G. J. Wasserburg, Lunar Planet. Sci. XII (1981) 1115.

[249] S. V. S. Murty, J. N. Goswami, Y. A. Shukolyukov, Ap. J. 475 (1997) L65.

[250] V.K. Rai, S.V.S. Murty, \& U. Ott, Geochim. Cosmochim. Acta 67 (2003) 4435.

[251] Y. Lin, Y. Guan, L. A. Leshin, Z. Ouyang and D. Wang, Lunar Planet. Sci. 35 (2004) 2084.

[252] Y. Lin, Y. Guan, L. A. Leshin, Z. Ouyang and D. Wang, Proc. Nat. Acad. Sci. 102, (2005) 1306.

[253] R. Reifarth, K. Schwarz and F. Käppeler, Ap. J. 528 (2000) 573. 
[254] K. Lodders, Ap. J. 591 (2003) 1220.

[255] R. Mauersberger, U. Ott, C. Henkel, J. Cernicharo, and R. Gallino, Astron. Astrophys. 426 (2004) 219.

[256] K. K. Marhas and J. N. Goswami, New Astron. Rev. 48 (2004) 139.

[257] I. Leya, A. N. Halliday, R. Wieler, Ap. J. 594 (2003) 605.

[258] J. N. Goswami, K. K. Marhas and S. Sahijpal, Ap. J. 549 (2001) 1151.

[259] S. J. Desch, H. C. Connolly, Jr. and G. Srinivasan, Ap. J. 602 (2004) 528.

[260] K. K. Marhas, J. N. Goswami and A.M. Davis, Meteorit. Planet. Sci. 37 (2002) A94.

[261] K. K. Marhas and J. N. Goswami, Lunar Planet. Sci. XXXIV (2003) 1303.

[262] K. K. Marhas, J. N. Goswami and A.M. Davis, Science 298 (2002) 2182.

[263] F. H. Shu, H. Shang, A. E. Glassgold, T. Lee, Science 277 (1997) 1475.

[264] F. H. Shu, H. Shang, M. Gounelle, A. E. Glassgold, T. Lee, Ap. J. 548 (2001) 1029.

[265] M. Gounelle, F. H. Shu, H. Shang, A. E. Glassgold, K. E. Rehm and T. Lee, Ap. J. 548 (2001) 1051.

[266] C. Vockenhuber, F. Oberli, M. Bichler, et al., Phys. Rev. Lett. 93 (2004) 172501.

[267] S. B. Jacobsen, Annu. Rev. Earth Planet. Sci. 33 (2005) 531.

[268] G. Srinivasan, M. J. Whitehouse, I. Weber and A. Yamaguchi, Lunar Planet. Sci. 35 (2004) 1709.

[269] Q.-Z. Yin, S. B. Jacobsen, W. F. McDonough, I. Horn, M. I. Petaev and J. Zipfel, Ap. J. 536 (2000) L49.

[270] C. Münker, S. Weyer, K. Mezger, M. Rehkämper, F. Wombacher and A. Bischoff, Science 289 (2000) 1538.

[271] M Schönbächler, M. Rehkämper, A. N. Halliday et al. Science 295 (2002) 1705.

[272] J. F. Minster and C. J. Allègre, Geochim. Cosmochim. Acta 46 (1982) 565.

[273] S. Goriely and M. Arnould, Astron. Astrophys. 379 (2001) 1113.

[274] J. J. Cowan, B. Pfeiffer, K.-L. Kratz et al., Ap. J. 521 (1999) 194.

[275] F.-K. Thielemann, P. Hauser, E. Kolbe et al., Space Sci. Rev. 100 (2002) 277.

[276] V. Hill, B. Plez, R. Cayrel, T.C. Beers, in Semaine de l'Astrophysique Française, Eds. F. Combes, D. Barret, F. Thévenin, (EdP-Sciences: 2001) 205.

[277] P. A. Seeger, W. A. Fowler and D. D. Clayton, Ap. J. Suppl. Ser. 11 (1965) 121. 
[278] R. E. Lingenfelter, J. C. Higdon, K.-L. Kratz and B. Pfeiffer, Ap. J. 591 (2003) 228.

[279] G. Turner, T. M. Harrison, G. Holland, S. Mojzsis and J. Gilmour, Science 306 (2004) 89.

[280] D. Porcelli and G. J. Wasserburg, Geochim. Cosmochim. Acta 59 (1995) 4921.

[281] B. Hagee, T. J. Bernatowicz, F. A. Podosek, M. L. Johnson, D. S. Burnett and M. Tatsumoto, Geochim. Cosmochim. Acta 54 (1990) 2847.

[282] D. S. Burnett, M. I. Stapanian, J. H. Jones, in Essays in Nuclear Astrophysics, ed. C. A. Barnes, D. D. Clayton and D. N. Schramm (Cambridge Univ. Press: New York, 1982) 141.

[283] J. H. Jones and D. S. Burnett, Geochim. Cosmochim. Acta 51 (1987) 769.

[284] J. B. Blake and D. N. Schramm, Nature Phys. Sci. 243 (1973) 138.

[285] J. H. Chen, G. J. Wasserburg and D. A. Papanastassiou, Lunar Planet. Sci. 23 (1992) 223.

[286] J. H. Chen, G. J. Wasserburg and D. A. Papanastassiou, Lunar Planet. Sci. 24 (1993) 277.

[287] E. D. Feigelson, G. D. Garmire \& S. H. Pravdo, Ap. J. 572 (2002) 335.

[288] R. Cayrel, M. Spite, F. Spite et al., ASP Conf. Series 245 (2001) 244.

[289] S. Amari, L. R. Nittler, E. Zinner, K. Lodders, R. S. Lewis, Ap. J. 559 (2001) 463.

[290] M. Lugaro, A.M. Davis, R. Gallino, M.J. Pellin, O. Straniero, F. Käppeler, Ap. J. 593 (2003) 486.

[291] N. Krestina, W. Hsu and G. J. Wasserburg, Lunar Planet. Sci. XXXIII (2002) 1425. 